\title{
Calculation of atmospheric neutrino flux using the interaction model calibrated with atmospheric muon data
}

\author{
M. Honda* and T. Kajitat \\ Institute for Cosmic Ray Research, the University of Tokyo, \\ 5-1-5 Kashiwa-no-ha, Kashiwa, Chiba 277-8582, Japan \\ K. Kasaharat \\ Shibaura Institute of Technology, 307 Fukasaku, \\ Minuma-ku, Saitama 330-8570, Japan \\ S. Midorikawa \\ Faculty of Software and Information Technology, \\ Aomori University, 2-3-1 Kobata, Aomori 030-0943, Japan. \\ T. Sanuki \\ International Center for Elementary Particle Physics, \\ the University of Tokyo, 7-3-1 Hongo, \\ Bunkyo-ku, Tokyo 113-0033, Japan
}

(Dated: February 5, 2008) 


\begin{abstract}
Using the "modified DPMJET-III" model explained in the previous paper [1], we calculate the atmospheric neutrino flux. The calculation scheme is almost the same as HKKM04 [2], but the usage of the "virtual detector" is improved to reduce the error due to it. Then we study the uncertainty of the calculated atmospheric neutrino flux summarizing the uncertainties of individual components of the simulation. The uncertainty of $K$-production in the interaction model is estimated using other interaction models: FLUKA'97 and Fritiof 7.02, and modifying them so that they also reproduce the atmospheric muon flux data correctly. The uncertainties of the flux ratio and zenith angle dependence of the atmospheric neutrino flux are also studied.
\end{abstract}

PACS numbers: 95.85.Ry, 13.85.Tp, 14.60.Pq

*mhonda@icrr.u-tokyo.ac.jp; http://icrr.u-tokyo.ac.jp/ mhonda

kajita@icrr.u-tokyo.ac.jp

kasahara@icrc.u-tokyo.ac.jp

\$midori@aomori-u.ac.jp

qsanuki@icepp.s.u-tokyo.ac.jp 


\section{INTRODUCTION}

In the previous paper [1] (hereafter Paper I), we have studied the interaction model (DPMJET-III [3]) employed in the HKKM04 atmospheric neutrino flux calculation [2], using the atmospheric muon flux data observed by precision measurements [4, 5, 6]. In the study, we found that the calculated and observed muon fluxes did not agree, especially for momenta above $30 \mathrm{GeV} / \mathrm{c}$. We modified the interaction model to improve the agreement between the calculated and observed atmospheric muon fluxes. We call this modified interaction model the modified DPMJET-III in this paper. Note, the modification is actually applied to the "inclusive DPMJET-III" in a phenomenological way based on the quark-parton model.

In this paper, we calculate the atmospheric neutrino flux with the modified DPMJET-III (Sec. II). The calculation scheme and the physical input data are basically the same as the HKKM04. However, we update the geomagnetic model from IGRF2000 to IGRF2005 [7], and improve the use of "virtual detector" in the 3-dimensional calculation to reduce the error due to it [8].

There are other hadronic interaction models which are used in the detector simulations of high energy experiments, such as FLUKA'97 [9] and Fritiof 7.02 [10]. We calculate the atmospheric neutrino fluxes with these interaction models applying the modification, so that they also reproduce the atmospheric muon flux observed by the precision measurements (Sec. III). Note, to reproduce the observed muon flux, modifying the primary flux model might be alternative solution. We calculated the atmospheric neutrino flux, changing the spectral index of primary cosmic ray protons from -2.71 to -2.66 above $100 \mathrm{GeV}$, which also reproduces the observed muon flux in $\mu^{+}+\mu^{-}$sum correctly with the original DPMJET-III.

Those calculations give almost the same atmospheric neutrino flux in the energy region below $100 \mathrm{GeV}$, where $\pi$ 's are the main source of atmospheric neutrinos. With the modifications based on the atmospheric muon data, the $\pi$ production is almost the same in all three calculations. However the $K$ 's are not related to the atmospheric muons below $1 \mathrm{TeV} / \mathrm{c}$, and above this momenta, almost no muon flux is available from the precision measurements. There remain sizable differences in the $K$ production, resulting in differences in atmospheric neutrino fluxes at higher energies.

In Sec. IV, we estimate the uncertainties in our calculations. As the uncertainties of the predicted atmospheric neutrino flux is crucial for the study of neutrino oscillations, the 
study of them is important [11]. Since our calculation reproduces the observed atmospheric muon flux data, the uncertainty due to that in the $\pi$ production could be estimated from the experimental error and the residual of the reconstruction of atmospheric muon data. The uncertainty of $K$ production is estimated from the variation of the atmospheric neutrino flux at higher energies calculated in the modified calculation schemes. The total uncertainty is estimated by summarizing individual uncertainties. Note, the stabilities of the $\left(\nu_{\mu}+\right.$ $\left.\bar{\nu}_{\mu}\right) /\left(\nu_{e}+\bar{\nu}_{e}\right)$ ratio and the zenith angle dependence of the atmospheric neutrino flux are especially important in the study of neutrino oscillations. They are also studied with the uncertainty of the flux value.

\section{CALCULATION OF ATMOSPHERIC NEUTRINO FLUX}

In this section, we describe the calculation of the atmospheric neutrino flux with the modified DPMJET-III in detail. The calculation scheme is basically the same as HKKM04 [2]. For the primary flux model, we take the same primary flux model as HKKM04, based on AMS [12, 13] and BESS [4, 14] data, with a spectral index of -2.71 above $100 \mathrm{GeV}$ (see also Refs. [15, 16]). For the model of the atmosphere, we used the US-standard '76 [17], as the error due to the atmospheric density model is sufficiently small for the calculation of atmospheric neutrino flux [1]. Note, however, we update the geomagnetic field model from IGRF2000 to IGRF2005 [7], and improve of the usage of the "virtual detector" as explained in this section.

Note, there are a considerable number of 3-dimensional calculations of the atmospheric neutrino flux [8, 18, 19, 20, 21, 22, 23]. However, all those calculations suffer from the small statistics at higher neutrino energies $(\gtrsim 10 \mathrm{GeV})$, due to the inefficiency of the 3-dimensional calculation scheme. In this paper, we calculate the atmospheric neutrino flux averaged over all azimuthal angles, combining 3-dimensional and 1-dimensional calculations. In HKKM04, it is shown that the atmospheric neutrino flux calculated with a 1-dimensional scheme agrees with that calculated with the 3-dimensional scheme above a few $\mathrm{GeV}$, averaged over all azimuthal angles, although more than a few \% azimuth angle dependence remains in the atmospheric neutrino flux at $10 \mathrm{GeV}$ due to muon curvature in the geomagnetic field.

For the 3-dimensional calculation, we assume the surface of the Earth is a sphere with $R_{e}=6378.180 \mathrm{~km}$. We also assume three more spheres; the injection, simulation, and 
escape spheres. The radius of the injection sphere is taken as $R_{i n j}=R_{e}+100 \mathrm{~km}$, the simulation sphere as $R_{\text {sim }}=R_{e}+3000 \mathrm{~km}$, and the escape sphere as $R_{e s c}=10 \times R_{e}$. Note, a spheroid with an eccentricity of $\sim 1 / 298$ is a better approximation for the Earth. However, the differece from the sphare approximation is small, and we estimate the errors of the atmospheric neutrino flux due to the sphere approximation is also small $(\lesssim 1 \%)$.

Cosmic rays are sampled on the injection sphere uniformly toward the inward direction, following the given primary cosmic ray spectra. Before they are fed to the simulation code for the propagation in air, they are tested to determine whether they pass the rigidity cutoff, i.e., the geomagnetic barrier. For a sampled cosmic ray, the 'history' is examined by solving the equation of motion in the negative time direction. When the cosmic ray reaches the escape sphere without touching the injection sphere again in the inverse direction of time, the cosmic ray can pass through the magnetic barrier following its trajectory in the normal direction of time.

The propagation of cosmic rays is simulated in the space between the surface of Earth and the simulation sphere. When a particle enters the Earth, it loses its energy very quickly, and results in neutrinos with energy less than $100 \mathrm{MeV}$. Therefore, we discard such particles as soon as they enter the Earth, as most neutrino detectors which observe atmospheric neutrinos do not have sensitivity below $100 \mathrm{MeV}$.

For secondary particles produced in the interaction of a cosmic ray and air-nucleus, there is the possibility that they go out from and re-enter in the atmosphere and create low energy neutrinos. Therefore, a simulation sphere which is too small may miss such secondary particles. On the other hand, it is very time consuming to follow all particles out to distances far from the Earth. In HKKM04, the simulation sphere with radius $R_{\text {sim }}=R_{e}+3000 \mathrm{~km}$ was found to be large enough to suppress the error to well below the $1 \%$ level.

Note, neutrino detectors are very small compared with the size of the Earth, and are considered as the infinitesimal points on the surface of the Earth. We introduce a finite size "virtual detector" for each targert detector in the 3-dimensional calculation scheme. In HKKM04, the surface of the Earth within a circle around the target detector of radius $\theta_{d}=10^{\circ}(\sim 1000 \mathrm{~km})$ is used as the virtual detector. When neutrinos pass throuth the surface of the Earth inside of the circle (upward or downward), they are registered. We do not need the virtual detector in 1-dimensional calculation scheme, since it treats the propagation of the cosmic rays on a line which go through the neutrino detector. This is a 
far less time consuming computation scheme than the 3-dimensional calculation scheme for the atmospheric neutrino flux,

The finite size of the virtual detector introduces an error, since it averages the neutrino flux over positions where the geomagnetic conditions are different from the position of target detector [8]. To study the relation between the size and the error, we calculate the atmospheric neutrino flux with different size virtual detectors, $\theta_{d}=10^{\circ}\left(\Phi_{\nu}\left(10^{\circ}\right)\right)$ and $\theta_{d}=5^{\circ}$ $\left(\Phi_{\nu}\left(5^{\circ}\right)\right)$. The fluxes $\Phi_{\nu}\left(10^{\circ}\right)$ and $\Phi_{\nu}\left(5^{\circ}\right)$ are compared in Fig. 1 for Kamioka from the HKKM04 calculation averaging over all azimuthal angles. We find a difference is seen for downward directions, and is almost constant for $\cos \theta_{z}>0$ in ratio, where $\theta_{z}$ is the zenith angle of the arrival direction of the neutrinos. Therefore we expect the maximum error at $E_{\nu}=0.1 \mathrm{GeV}$ is $\sim 5 \%$ for downward directions averaging over azimuthal angles. The error due to the finite size virtual detector are smaller than those due to uncertainty of hadronic interaction model in HKKM04.
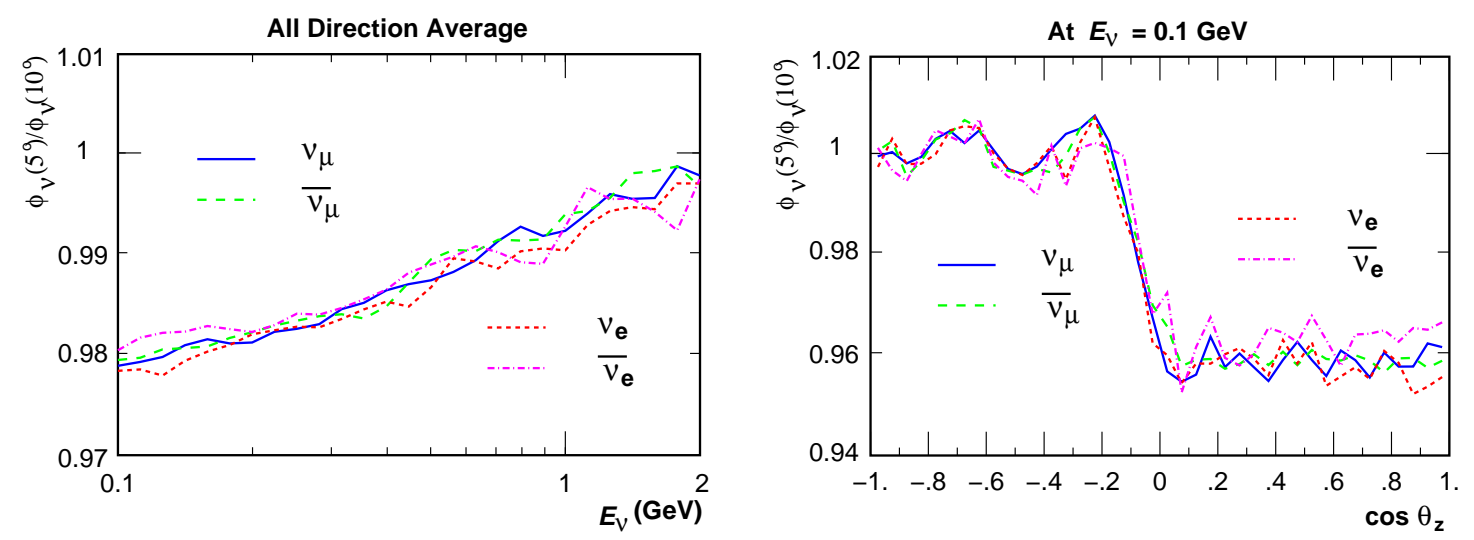

FIG. 1: Left panel: Ratio of all direction averaged flux with a smaller virtual detector $\left(\Phi_{\nu}\left(\theta_{d}=5^{\circ}\right)\right)$ to that with the larger virtual detector $\left(\Phi_{\nu}\left(\theta_{d}=10^{\circ}\right)\right)$ used in HKKM04. Right panel: Zenith angle $\left(\theta_{z}\right)$ dependence of the ratio at $E_{\nu}=0.1 \mathrm{GeV}$ in azimuthal average.

We can reduce the error due to the finite size virtual detector, with a little more computation. Let us assume the "true" atmospheric neutrino flux is expressed as an analytic function of the position. Note, we drop the arguments for arrival direction in the following expressions. The discussion here should apply to each arrival direction independently. We consider the power expansion the analytic function as,

$$
\phi_{\nu}\left(\theta_{x}, \theta_{y}\right)=\phi_{\nu}^{(0,0)}+\phi_{\nu}^{(1,0)} \cdot \theta_{x}+\phi_{\nu}^{(0,1)} \cdot \theta_{y}+\phi_{\nu}^{(2,0)} \cdot \theta_{x}^{2}+\phi_{\nu}^{(1,1)} \cdot \theta_{x} \theta_{y}+\phi_{\nu}^{(0,2)} \cdot \theta_{y}^{2}+\cdots
$$


and

$$
\left.\phi_{\nu}^{(m, n)} \equiv \frac{1}{m ! \cdot n !} \cdot \frac{\partial^{m+n} \phi_{\nu}}{\partial \theta_{x}^{m} \partial \theta_{y}^{n}}\right|_{\left(\theta_{x}, \theta_{y}\right)=(0,0)},
$$

where, $\theta_{x}, \theta_{y}$ are the distances from the target detector in center angle to any directions perpendicular to each other, say, to South and East respectively, i.e., $\left(\theta_{x}, \theta_{y}\right)$ constitute a local coordinate system.

In the Monte Carlo calculation of the atmospheric neutrino flux, the calculated flux with a finite size virtual detector is the average flux over the virtual detector. With the increase of statistics, the flux $\Phi\left(\theta_{d}\right)$ calculated in Monte Carlo calculation should approach

$$
\frac{1}{S\left(\theta_{d}\right)} \int_{\theta_{r}<\theta_{d}} \phi_{\nu}\left(\theta_{x}, \theta_{y}\right) d \theta_{x} d \theta_{y} \quad\left(\theta_{d} \ll 1\right),
$$

where $S\left(\theta_{d}\right) \simeq \pi \theta_{d}^{2}$ is the "area" of the virtual detector, and $\theta_{r} \simeq \sqrt{\theta_{x}^{2}+\theta_{y}^{2}}$. The integrations of terms proportional to $\theta_{x}$ or $\theta_{y}$ in Eq. 1 vanish, and non-vanishing terms start from the integrations of second order terms, $\phi_{\nu}^{(2,0)} \theta_{x}^{2}+\phi_{\nu}^{(1,1)} \theta_{x} \theta_{y}+\phi_{\nu}^{(0,2)} \theta_{y}^{2}$, resulting in the terms proportional to $\theta_{d}^{4}$. For a sufficiently small $\theta_{d}, \Phi_{\nu}\left(\theta_{d}\right)$ is expressed as,

$$
\Phi_{\nu}\left(\theta_{d}\right) \simeq \Phi^{(0,0)}+\frac{\Phi^{(2)} \theta_{d}^{4}}{S\left(\theta_{d}\right)}=\Phi^{(0,0)}+\Phi^{\left(2^{\prime}\right)} \theta_{d}^{2}
$$

where $\Phi^{\left(2^{\prime}\right)} \equiv \Phi^{(2)} \theta_{d}^{2} / S\left(\theta_{d}\right) \simeq \Phi^{(2)} / \pi$. When we have the neutrino fluxes calculated with two virtual detectors with small enough radii $\theta_{d}$ and $\theta_{d} / 2$ for the same target, we expect $\Phi_{\nu}\left(\theta_{d}\right)-\Phi_{\nu}\left(\theta_{d} / 2\right) \simeq \Phi^{\left(2^{\prime}\right)} \cdot\left[\theta_{d}^{2}-\left(\theta_{d} / 2\right)^{2}\right]$, then $\Phi_{\nu}(0)$, the true flux value at the target detector, is given as

$$
\Phi_{\nu}(0)=\Phi^{(0,0)} \simeq \Phi_{\nu}\left(\theta_{d}\right)-\frac{4}{3} \cdot\left[\Phi_{\nu}\left(\theta_{d}\right)-\Phi_{\nu}\left(\theta_{d} / 2\right)\right] .
$$

As is seen in Fig. 1, the difference of the $\Phi_{\nu}\left(10^{\circ}\right)$ and $\Phi_{\nu}\left(5^{\circ}\right)$ is almost constant for $\cos \theta_{z}>0$, so it should be sufficient to examine the assumption and procedure for vertical down going directions. In the left panel of Fig. 2, we plotted the total neutrino flux for the vertical down going directions $\left(\cos \theta_{z}>0.9\right)$ calculated with different size of virtual detectors, $\theta_{d}=10^{\circ}, 5^{\circ}$, and $2.5^{\circ}$ for Kamioka, Sudbury, and Gran Sasso with the HKKM04 calculation. In the right panel of Fig. 2, we depicted the difference to the estimated true value with Eq. 5 in the ratio. We may say the convergence of the calculated fluxes to the "true value" agrees with the expectation of Eq. 4, and we apply the Eq. 5 with $\theta_{d}=10^{\circ}$ and $5^{\circ}$ to the atmospheric neutrino flux calculated in the 3-dimensional scheme. Note, the error due to the finite size of virtual detector does not exist in the 1-dimensional calculation scheme. 

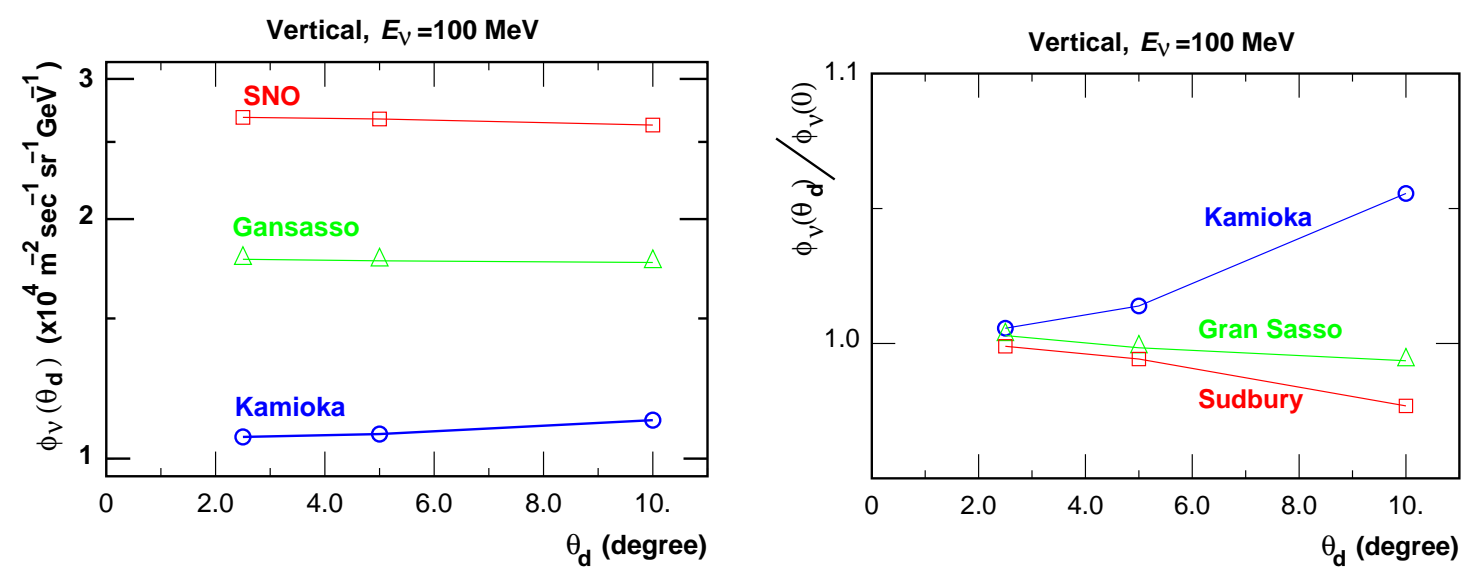

FIG. 2: Left: Atmospheric neutrino fluxes for vertical directions calculated with the virtual detectors with different radii. Right: Ratio of fluxes calculated with virtual detectors with $\theta_{d}=10,5$, and $2.5^{\circ}$ to the flux estimated with Eq. 5 .

Thus calculated atmospheric neutrino fluxes in the 3-dimensional scheme are shown in the Appendix A up to $10 \mathrm{GeV}$ for Kamioka, Sudbury, and Gran Sasso separately. The neutrino flux calculated for the Soudan2 site is almost identical to that calculated for Sudbury. The neutrino flux calculated for Frejus is $\sim 10 \%$ larger than that for Gran Sasso at $0.1 \mathrm{GeV}$, and the difference is smaller at higher energies. Above $10 \mathrm{GeV}$, the atmospheric neutrino flux is calculated using the 1-dimensional scheme. They are tabulated in the Appendix B up to $10 \mathrm{TeV}$.

We compared the atmospheric neutrino fluxes calculated with modified and original DPMJET-III in the ratio of flux values in Fig 3, averaging over all directions for Kamioka up to $1 \mathrm{TeV}$. The atmospheric neutrino flux calculated with the modified DPMJET-III shows an increase above $10 \mathrm{GeV}$ from that with the original DPMJET-III, but the increase rate is different for different kinds of neutrinos. This is because the modification of the interaction model in Paper I enhances the productions of $\pi^{ \pm}$'s, $K^{+}$'s, and $K^{0}$, with no change for $K^{-}$ production. Therefore, the increase of $\nu_{\mu}$ and $\nu_{e}$ is larger than that of $\bar{\nu}_{\mu}$ and $\bar{\nu}_{e}$.

In Fig 4, we compared the atmospheric neutrin fluxes calculated with the modified and original DPMJET-III with those from other calculations based on the 3-dimensional calculation scheme, Bartol [8, 24] and Fluka [19, 20]. The atmospheric neutrino fluxes calculated for Kamioka and averaged over all the directions are depicted in panel (a) and the ratios are compared in panel (b) up to $1 \mathrm{TeV}$. Although there are sizable differences in the flux values 


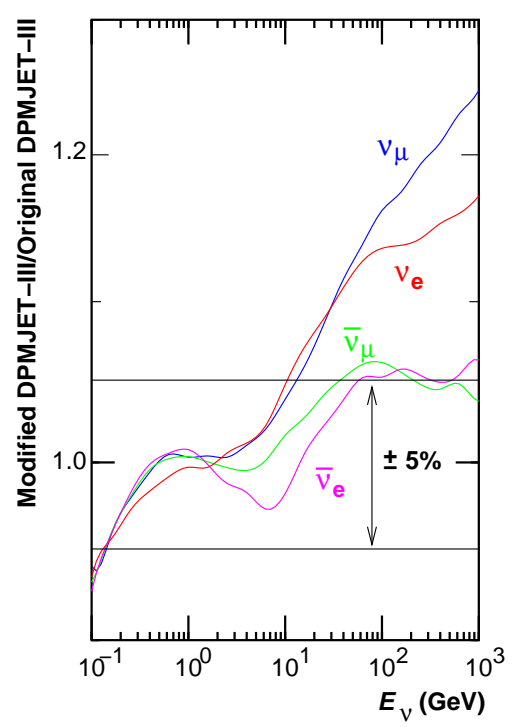

FIG. 3: The comparison of the atmospheric neutrino fluxes calculated with modified and original DPMJET-III in ratio. The denominator is the original DPMJET-III.

among different calculations, the ratio $\left(\nu_{\mu}+\bar{\nu}_{\mu}\right) /\left(\nu_{e}+\bar{\nu}_{e}\right)$ is almost identical to each other below $100 \mathrm{GeV}$ among them.
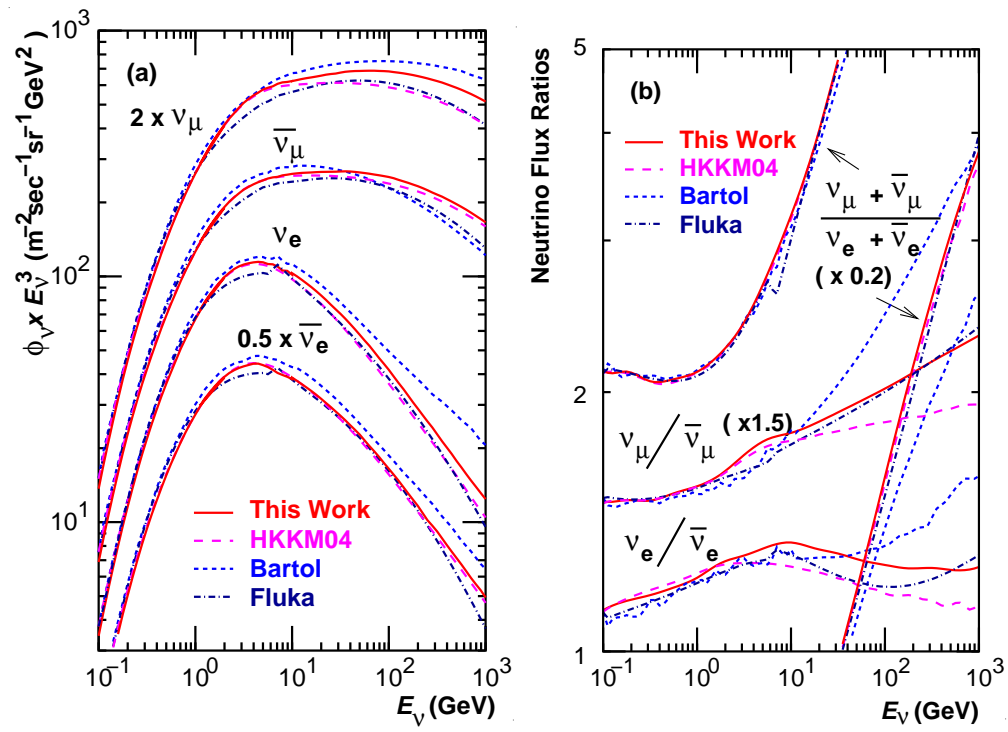

FIG. 4: The comparison of all direction average of the atmospheric neutrino fluxes with other calculations [8, 19, 20, 24]; (a) the absolute values of each kind of neutrinos and (b) the ratio of them. 


\section{MODIFICATION OF FLUKA'97 AND FRITIOF 7.02}

In this section, we modify the FLUKA'97 and Fritiof 7.02 interaction models, so that they reproduce the observed atmospheric muon data, following the procedure we used to modify DPMJET-III in Paper I. Then we calculate the resulting atmospheric neutrino flux to study the robustness of the modification procedure. The calculations in this section are carried out using the 1-dimensional calculation scheme for computation speed. The modification is applied to the hadronic interactions above $30 \mathrm{GeV}$, to study the muon flux above $10 \mathrm{GeV} / \mathrm{c}$ and neutrino flux above $3 \mathrm{GeV} / \mathrm{c}$.

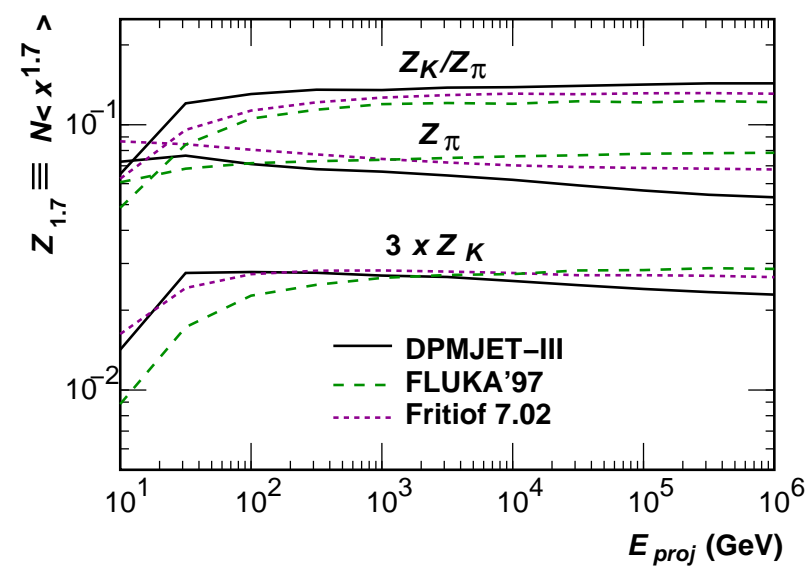

FIG. 5: $Z$-factors for $\pi^{+}+\pi^{-}\left(Z_{\pi}\right)$ and $K^{+}+K^{-}\left(Z_{K}\right)$, and their ratio in each interaction model as the function of projectile energy. Solid lines are for DPMJET-III, dashed lines for FLUKA'97, and dotted lines for Fritiof 7.02.

The secondary spectra of $\pi$ and $K$ productions differ between interaction models. The difference is seen in the $Z$-factors defined as

$$
Z_{i} \equiv N_{i}<x_{i}^{1.7}>\text { and } x \equiv \frac{p_{i}}{p_{\text {proj }}}
$$

where $N_{i}$ is the multiplicity and $p_{i}$ is the momentum of the $i$ secondary particle, and $p_{\text {proj }}$ is the projectile momentum. The $Z$-factors are compared in the sums, $Z_{\pi^{+}}+Z_{\pi^{-}}$and $Z_{K^{+}}+Z_{K^{-}}$, for DPMJET-III, FLUKA'97, and Fritiof 7.02. in Fig. 5. Note, the contribution of neutral $K$ 's to the neutrino flux is smaller than that of the charged $K^{\prime}$ 's and $\pi^{0}$ does not contribute to either muon flux or neutrino flux. The neutral $\pi$ and $K$ are not compared in the figure. 

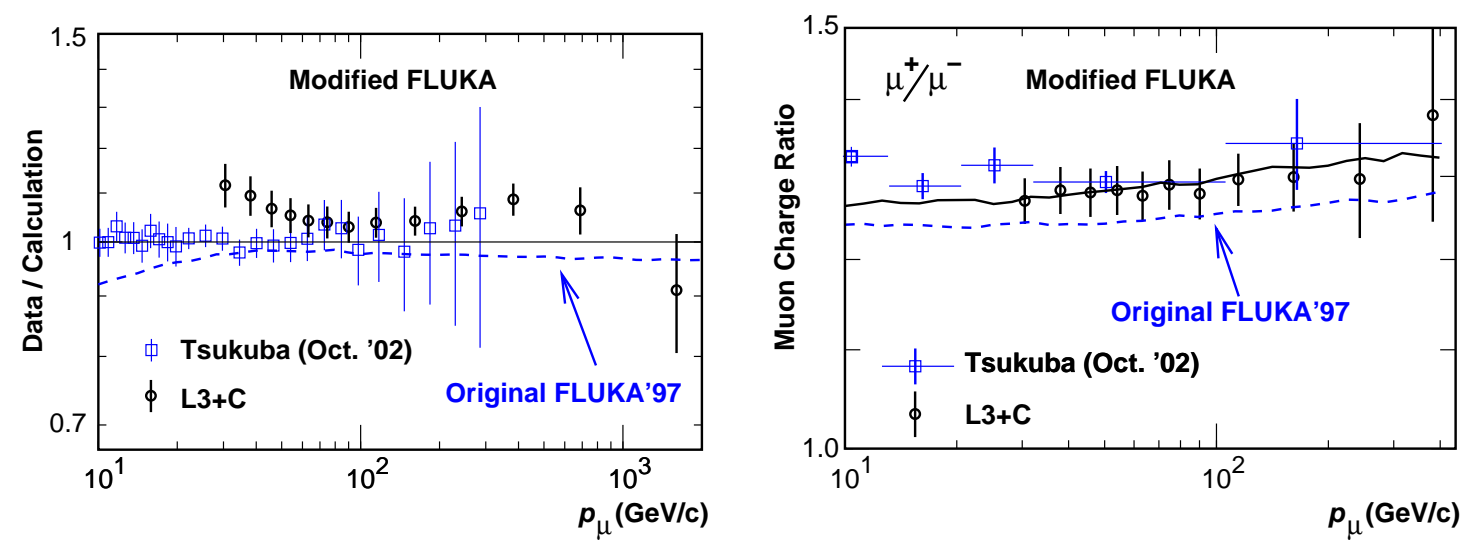

FIG. 6: Left: The comparison of muon flux data and the calculations. The observed data are shown as the ratios to the calculations with modified FLUKA. The dashed line shows the ratio of the calculation with the original FLUKA'97 to that with modified FLUKA. Right: Comparison of observed muon charge ratio with the calculations. The solid line shows the calculation with modified FLUKA and the dashed line the calculation with original FLUKA'97.

In Fig. 6, we show the comparison of observed muon fluxes and calculations with the original and modified FLUKA'97 interaction models. We find the modification clearly improves the agreement of the observations and the calculation. The muon flux calculated with the original FLUKA'97 shows rather a better agreement than that of original DPMJET-III above $30 \mathrm{GeV} / \mathrm{c}$ (see also Fig. [8). However the muon flux reproduced by FLUKA'97 becomes increasingly smaller than the observation for momenta below $30 \mathrm{GeV} / \mathrm{c}$. This feature is also observed in the reconstruction of the muon flux for balloon altitudes [25], and FLUKA'97 was not used in HKKM04. Hereafter, we refer to this calculation scheme as the "modified FLUKA".

In Fig. 7, we show the comparison of observed muon fluxes and the calculations with the original and modified Fritiof 7.02 interaction models. We find here again the modification clearly improves the agreement of the observations and the calculation. The disagreement of the muon flux calculated with the original Fritiof 7.02 is larger than those with the original DPMJET-III or the original FLUKA'97. We find the modification improved the agreement so that it is almost as good as the modified DPMJET-III or modified FLUKA. Hereafter, we refer to this calculation scheme as the "modified Fritiof".

With the original DPMJET-III, we can reproduce the muon flux data in $\mu^{+}+\mu^{-}$sum by the modification of the primary flux model changing of the spectral index from -2.71 

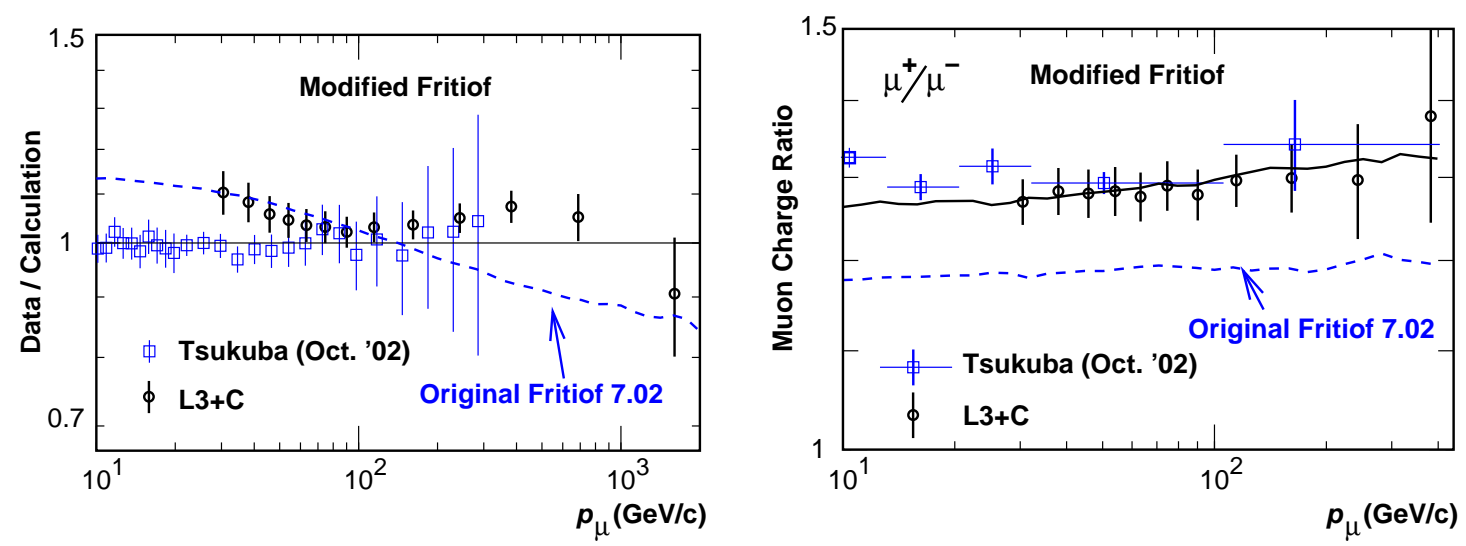

FIG. 7: Left: The comparison of muon flux data and the calculations. The observed data are shown as the ratios to the calculations with modified Fritiof. The dashed line shows the ratio of the calculation with the original Fritiof 7.02 to that with modified Fritiof. Right: Comparison of observed muon charge ratio with the calculations. The solid line shows the calculation with modified Fritiof and the dashed line the calculation with original Fritiof 7.02.

to -2.66 above $100 \mathrm{GeV}$ [26]. However, with only the modification of the primary flux model, it is difficult to reproduce the observed muon charge ratio. In terms of $Z$-factor, we find $Z_{\pi^{+}} / Z_{\pi^{-}}$must be $\sim 1.5$ at $1 \mathrm{TeV}$ to reproduce the observed muon charge ratio at $100 \mathrm{GeV}$, while $Z_{\pi^{+}} / Z_{\pi^{-}}$is $\sim 1.35$ at $1 \mathrm{TeV}$ in the original DPMJET-III. We calculate the atmospheric muon flux with this modified primary flux model, also applying a light modification for DPMJET-III to reproduce the observed muon charge ratio, by increasing the ratio $Z_{\pi^{+}} / Z_{\pi^{-}}$to $\sim 1.5$. The calculated results are compared with the observation in Fig. 8. We find again the agreement of calculation and observation are equally as good as the other modified calculations. Hereafter, we will refer to this calculation scheme simply as the "modified primary flux".

In Fig. 9, we plotted the $Z_{\pi^{+}}+Z_{\pi^{-}}$and $Z_{K^{+}}+Z_{K^{-}}$for the interaction models modified as explained above. In addition, we plotted the values of the original DPMJET-III multiplying $E_{\text {proj }}^{0.05}$ above $100 \mathrm{GeV}$, to compare the modified primary flux scheme in terms of the $Z$ factor. We find all the modified calculations show good agreement in $Z_{\pi}$ in $30 \mathrm{GeV} \lesssim$ $E_{\text {proj }} \lesssim 10 \mathrm{TeV}$, due to the adjustment of the $\pi$ productions with the muon flux data. The $Z_{K}$ 's are also closer to each other in the modified calculations. However, they still show large variations, even in $30 \mathrm{GeV} \lesssim E_{\text {proj }} \lesssim 10 \mathrm{TeV}$. The difference of $Z_{K}$ 's results from the original interaction models, and may be considered as the reasonable variation of $K$ 

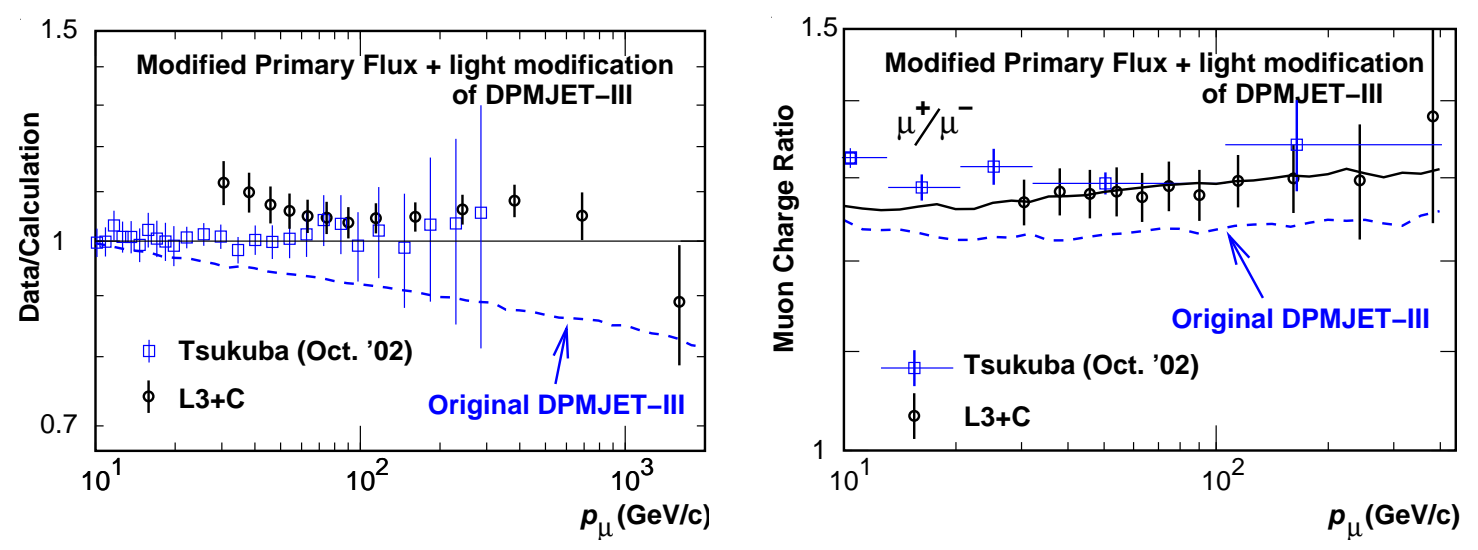

FIG. 8: Left: The comparison of muon flux data and calculations. The observed data are shown as the ratios to the calculations in modified primary flux scheme. The dashed line shows the ratio of calculation in original HKKM04 scheme to that in modified primary flux scheme. Right: The comparison of muon charge ratio between observed data and the calculated in modified primary flux scheme. The solid line shows the calculation with modified primary flux scheme and the dashed line the calculation in original HKKM04 scheme. Note, in the modified primary flux scheme, a modification is applied to DPMJET-III to reproduce the observed muon charge ratio. For the details, see the text.

productions after the modification.

Now we calculate the atmospheric neutrino flux with the modified calculation schemes. The calculations are carried out in the 1-dimensional scheme, and the results are compared with those with modified DPMJET-III in Fig. 10 above $3 \mathrm{GeV}$. Note, we also depicted the comparison of the calculations with different atmosphere models defined as,

$$
\rho_{u s, \varepsilon}(h)=\frac{1}{1+\varepsilon} \cdot \rho_{u s}\left(\frac{h}{1+\varepsilon}\right) \text {, }
$$

where $\rho_{u s}(h)$ is the density profile of US-standard '76 [17]. This is the same modification of the US-standard '76, which we used in Sec. IV of Paper I to study the effect of the atmospheric density profile on the lepton fluxes. We take the parameter $\varepsilon$ to be \pm 0.05 as in that study, and use the modified DPMJET-III for the interaction model.

In the left panel of Fig. 10, we find the atmospheric neutrino fluxes calculated with the modified calculations agree within $\pm 5 \%$ below $100 \mathrm{GeV}$. The agreement is considered to be due to the large contribution of $\pi$ 's in this energy region. Note, the different atmospheric models result in almost the largest difference for $\nu_{e}$ and $\bar{\nu}_{e}$, and the difference for $\nu_{\mu}$ and $\bar{\nu}_{\mu}$ 


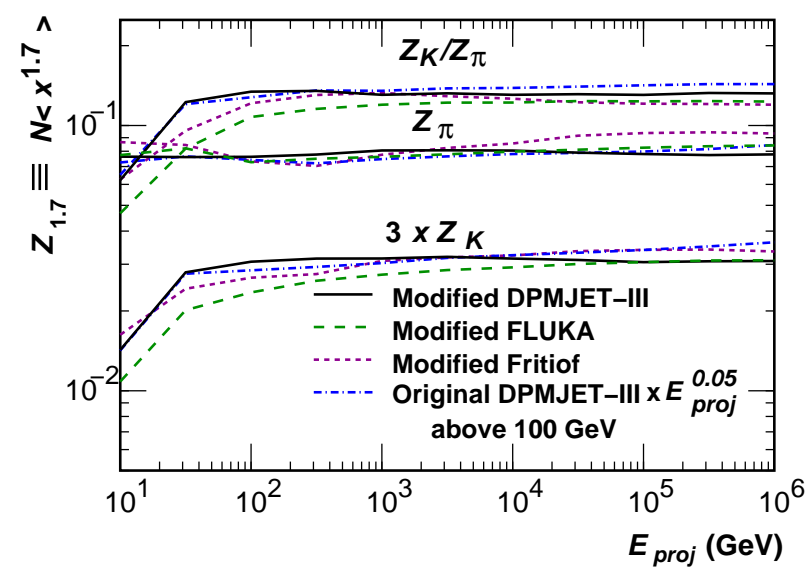

FIG. 9: $Z$-factors for $\pi^{+}+\pi^{-}\left(Z_{\pi}\right)$ and $K^{+}+K^{-}\left(Z_{K}\right)$, and their ratio in the modified interaction models as the function of projectile energy. Solid lines are for DPMJET-III, dashed lines for FLUKA'97, and dotted lines for Fritiof 7.02. We also plotted the values of original DPMJET-III multiplying $E_{\text {proj }}^{0.05}$ above $100 \mathrm{GeV}$, to compare the modified primary flux scheme in terms of the $Z$-factor.

is smaller than other modified calculations. This is a result of differences in $\pi-\mu$ successive decay and the muon propagation in the atmosphere.

Above $100 \mathrm{GeV}$, where the contribution of $K$ 's becomes large, we find a large variation of neutrino fluxes for all kinds of neutrino except for the $\nu_{\mu}$ flux. In our modification scheme, the $K^{+}$productions are modified at the same rate as the $\pi^{+}$productions. Therefore, the variation of $\nu_{\mu}$ flux is relatively small even above $100 \mathrm{GeV}$. Note, the modified primary flux scheme produces the largest neutrino fluxes among all the modified calculations and for all kinds of neutrino except for the $\nu_{\mu}$ above $100 \mathrm{GeV}$. This is due to the increase of $K^{-}$productions by the increase of primary cosmic ray in this model. In our modification scheme, the $K^{-}$productions are not altered.

In the right panel of Fig. 10, we show the neutrino flux ratios, $\nu_{\mu} / \bar{\nu}_{\mu}, \nu_{e} / \bar{\nu}_{e}$, and $\left(\nu_{\mu}+\right.$ $\left.\bar{\nu}_{\mu}\right) /\left(\nu_{e}+\bar{\nu}_{e}\right)$, averaged over all directions. We find the largest difference in the $\nu_{\mu} / \bar{\nu}_{\mu}$ ratio, and it could be explained by the differences in $\nu_{\mu} /$ and $\bar{\nu}_{\mu}$ seen in the left panel, and by the $K$-productions at higher energies. The difference of the ratios in the $3 \sim 100 \mathrm{GeV}$ range is much less than $\pm 5 \%$ for all ratios, where the contribution of $\pi$ 's is important. Especially, the difference in $\left(\nu_{\mu}+\bar{\nu}_{\mu}\right) /\left(\nu_{e}+\bar{\nu}_{e}\right)$ ratio is small at all the energies shown here $(\lesssim 2 \%)$. The largest variation in the $\left(\nu_{\mu}+\bar{\nu}_{\mu}\right) /\left(\nu_{e}+\bar{\nu}_{e}\right)$ ratio is found with the change of atmospheric 

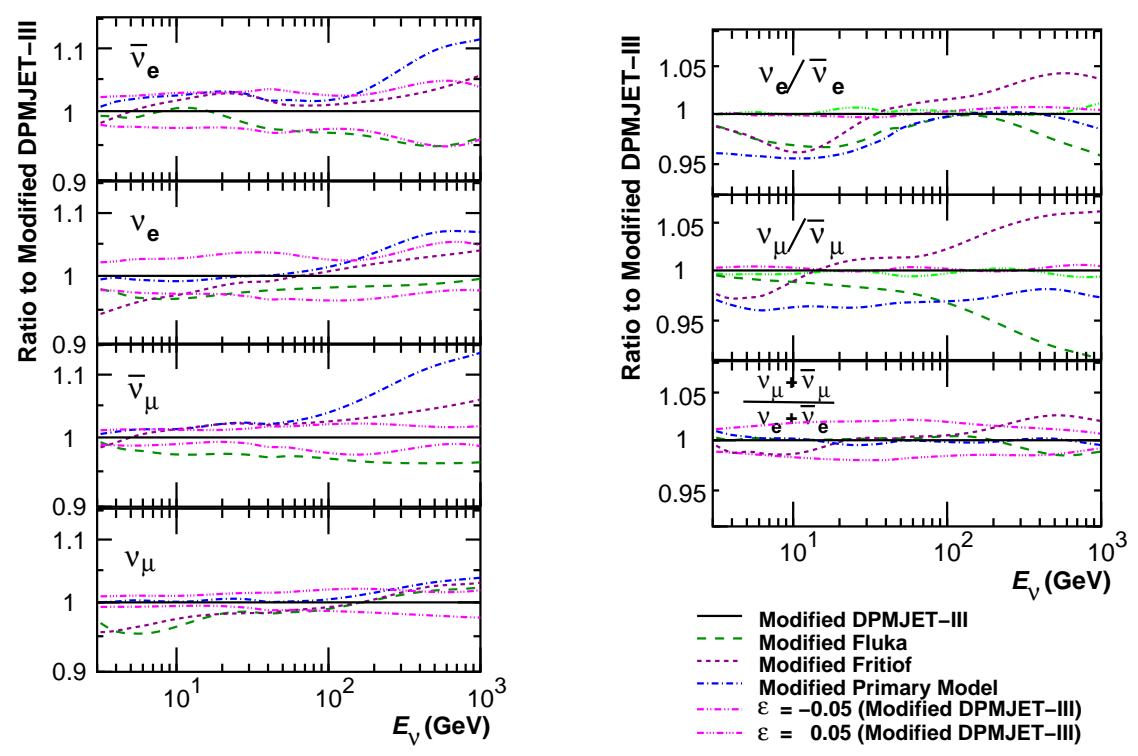

FIG. 10: Left panel: comparison of the atmospheric neutrino fluxes calculated in the different schemes. The atmospheric neutrino fluxes are averaged over all directions and the ratios to that of modified DPMJET-III are shown. The solid lines are for the modified DPMJET-III, the dashed lines for the modified FLUKA, the dotted lines for the modified Fritiof, and the dash-dot lines for the modified primary flux. Right panel: comparison of neutrino ratios, $\nu_{\mu} / \bar{\nu}_{\mu}, \nu_{e} / \bar{\nu}_{e}$, and $\left(\nu_{\mu}+\bar{\nu}_{\mu}\right) /\left(\nu_{e}+\bar{\nu}_{e}\right)$. The neutrino ratios are calculated for all direction averaged flux in each calculation scheme, and the ratio to the modified DPMJET-III shown. In addition, we show the ratios calculated with a slightly different atmospheric model with $\varepsilon= \pm 0.05$ in Eq. 7 .

model. This is the direct consequence of the large variation of $\nu_{e}$ and $\bar{\nu}_{e}$ and small variation $\nu_{\mu}$ and $\bar{\nu}_{\mu}$ resulting from the change in the atmospheric model.

\section{UNCERTAINTY IN THE ATMOSPHERIC NEUTRINO FLUX CALCULA- TION}

\section{A. Uncertainty for flux value of the neutrinos.}

Here, we estimate the total uncertainty or the total possible errors in the calculation of atmospheric neutrino flux. It may be expressed as

$$
\delta_{\text {tot }}^{2}=\delta_{\pi}^{2}+\delta_{K}^{2}+\delta_{\sigma}^{2}+\delta_{\text {air }}^{2}+\left(\delta_{\text {scheme }}^{2}+\delta_{\text {stat }}^{2}+\cdots\right),
$$


where $\delta_{\pi}$ is the uncertainty due to the uncertainty of $\pi$ production in the hadronic interaction model, $\delta_{K}$ is due to the $K$ production, $\delta_{\sigma}$ due to the hadronic interaction cross sections, $\delta_{\text {air }}$ due to the atmospheric density profile, $\delta_{\text {scheme }}$ due to the calculation scheme including any bugs in the code, and $\delta_{\text {stat }}$ due to statistical errors. The solar modulation of the cosmic rays and mountains above the neutrino detector cause sizable effects on the atmospheric neutrino flux. However, they are not a true uncertainty and they are included in our calculation correctly.

The statistical error in the Monte Carlo study is smaller than $1 \%$ below $3 \mathrm{TeV}$ and $3 \%$ below $10 \mathrm{TeV}$ for $\nu_{\mu}$ and $\bar{\nu}_{\mu}$. For $\nu_{e}$ and $\bar{\nu}_{\mu}$, it is a little worse and smaller than $1 \%$ below $10 \mathrm{GeV}, 3 \%$ below $3 \mathrm{TeV}$, and around $10 \%$ at $10 \mathrm{TeV}$. However, the statistical error is much smaller than those from other uncertainty sources. The largest error due to the calculation scheme is the finite size effect of the virtual detector, which we studied in detail in Sec. III. With the procedure proposed there, the remaining error would be much smaller than $1 \%$. We do not discuss $\delta_{\text {scheme }}$ and $\delta_{\text {stat }}$ in the following.

In Paper I, we have proven that the error of $\pi$ productions in the hadronic interaction model affects the atmospheric muon and neutrino fluxes produced by the $\pi$ decay at the same rate, namely

$$
\frac{\Delta \phi_{\mu}}{\phi_{\mu}} \simeq \frac{\Delta \phi_{\nu_{\mu}}}{\phi_{\nu_{\mu}}} \simeq \frac{\Delta \phi_{\nu_{e}}}{\phi_{\nu_{e}}}
$$

above $1 \mathrm{GeV}$. We may estimate $\delta_{\pi}$ from the comparison of the observation and calculation of the atmospheric muon flux. Since the atmospheric muon flux below $1 \mathrm{TeV}$ comes almost only from the $\pi$ decay, we use the sum of the experimental error and the residual of the reconstruction as the $\Delta \phi_{\mu}$ in Eq. 9 (see Fig. 15 of Paper I). Then we replace $\delta_{\pi}$ in Eq. 8 with $\left(\Delta \phi_{\mu} / \phi_{\mu}\right) \phi_{\nu}$, where $\phi_{\nu}$ is the sum of $\pi$ and $K$ contributions for a conservative estimation. The estimated uncertainty is depicted by the solid line above $1 \mathrm{GeV}$ in Fig. 11 .

For the $\delta_{K}$, we used the modified calculation schemes studied in Sec. III. We assumed the maximum neutrino flux difference from the modified DPMJET-III among them as $\delta_{K}$. The maximum difference for all kinds of neutrino for vertical direction is depicted by the dashed line in Fig. 11, since that variation is the largest of all zenith angles. Each difference is a little larger, but similar to that shown in the left panel of Fig. 10. Note, the maximum difference from the modified DPMJET-III is seen in the modified primary flux model in most of the cases.

For $\delta_{\sigma}$, we assumed the difference $\left|\Delta \phi_{\mu}-\Delta \phi_{\nu}\right|$ in the Fig. 10 of Paper I. Since the 
uncertainty of the interaction cross section works with opposing effects for atmospheric muons and neutrinos, the error of the interaction cross section introduces an error in the calibration of interaction model with the atmospheric muon flux data. On the other hand, as we use the observed atmospheric density profile, the calibration is not affected by the error of the atmospheric model. We use $\Delta \phi_{\nu}$ only in Fig. 9 of Paper I as the $\delta_{\text {air. All }}$ these uncertainties, $\delta_{\pi}\left(\delta_{\mu}\right), \delta \phi_{K}, \delta \phi_{\sigma}, \delta \phi_{\text {air }}$, and $\delta_{\text {tot }}$, are summarized in Fig. 11. Note, the estimations are conservative, and the maximum uncertainty is shown for all kind of neutrinos and zenith angles.

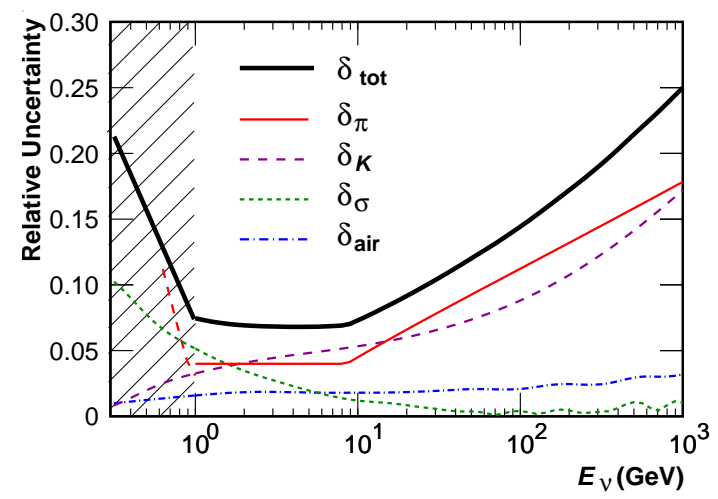

FIG. 11: The uncertainty of each error source for atmospheric neutrino flux and their sum with Eq. 8. Note, Eq. 9 loses its validity in the shaded region. The total error for $\lesssim 1 \mathrm{GeV}$ is estimated differently from Eq.8, as stated in the text. Note the statistical and systematic error are not shown in the figure.

We note, Eq. 9 is valid only for $\gtrsim 1 \mathrm{GeV}$. We have to estimate $\delta_{\pi}$ without using the atmospheric muon flux data at ground level. In Fig. 12, we show the study of the muon flux at balloon altitudes at Fort Sumner [27]. The modified DPMJET-III reproduces the muon flux within $\pm 10 \%$ at $\sim 1 \mathrm{GeV} / \mathrm{c}$, and $p_{\mu} / p_{\nu}$ ratio for the same momentum of parent $\pi$ 's remains $\sim 3$ even at the lower momenta, due to the small energy loss of muons at balloon altitudes. However, the distance of the production and observation places are longer than the muons observed at ground level. The muon decay in this distance make Eq. 9 less accurate for $\lesssim 1 \mathrm{GeV}$. We conservatively estimate $20 \%$ errors for pion productions responsible to the atmospheric neutrino at $\sim 0.3 \mathrm{GeV}$.

Note, the uncertainty studied above is for all the kind of neutrinos, and for all zenith angles. Limiting the kind of neutrino and the zenith angle, we may get a smaller estimation 

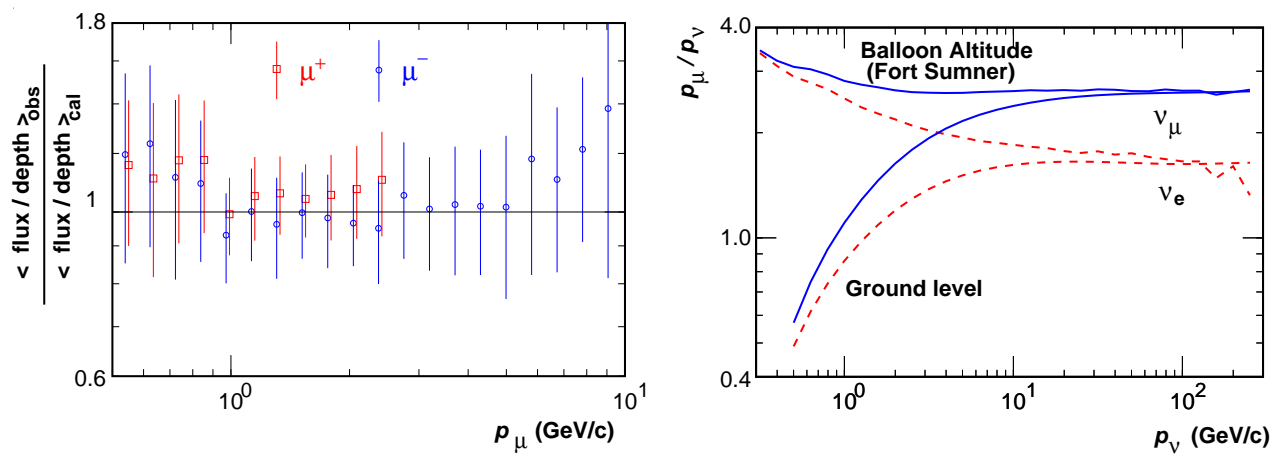

FIG. 12: The muon fluxes at balloon altitudes at Fort Sumner (Sept. 2001). Left: comparison calculated and observed muon fluxes. The dashed lines show $\pm 10 \%$ deviation from the calculated values. Right: $p_{\mu} / p_{\nu}$ ratio for the same momentum of parent $\pi$ 's in average. The dashed lines are the same quantities but for the muons at the ground level.

for the uncertainty. Especially, the uncertainties in the ratio of the different kind of neutrinos and the zenith angle dependence show smaller uncertainties. They are important in the study of the neutrino oscillations using the atmospheric neutrinos, and are studied in the following.

\section{B. Uncertainty for the flux ratio among different neutrinos}

We have studied the variation of the ratios, $\nu_{\mu} / \bar{\nu}_{\mu}, \nu_{e} / \bar{\nu}_{e}$, and $\left(\nu_{\mu}+\bar{\nu}_{\mu}\right) /\left(\nu_{e}+\bar{\nu}_{e}\right)$, among the modified calculations for all direction average in Sec. III, and shown in the right panel Fig. 10, We find the variation of the $\nu_{\mu} / \bar{\nu}_{\mu}$ and $\nu_{e} / \bar{\nu}_{e}$ is within $\pm 5 \%$ below $100 \mathrm{GeV}$, where $\pi$ 's are still the major source of atmospheric neutrinos. However, the variation increases above $100 \mathrm{GeV}$, due to the difference of the $K$ productions in the different models.

On the other hand, the ratio $\left(\nu_{\mu}+\bar{\nu}_{\mu}\right) /\left(\nu_{e}+\bar{\nu}_{e}\right)$ is very stable among different calculations especially below $100 \mathrm{GeV}(\lesssim 2 \%)$. Note, however, the variation of atmospheric model gives almost the largest variation to this ratio in this energy region. This is explained by the $\pi-\mu$ successive decay process:

$$
\begin{aligned}
\pi^{ \pm} \rightarrow & \mu^{ \pm}+\nu_{\mu}\left(\bar{\nu}_{\mu}\right) \\
& \rightarrow e^{ \pm}+\nu_{e}\left(\bar{\nu}_{e}\right)+\bar{\nu}_{\mu}\left(\nu_{\mu}\right)
\end{aligned}
$$

The decay and energy loss of muons are affected by the atmospheric density profile. When most of the muons decay in the atmosphere, the ratio $\left(\nu_{\mu}+\bar{\nu}_{\mu}\right) /\left(\nu_{e}+\bar{\nu}_{e}\right)$ becomes small. 
When muons lose energy at a larger rate, the products of muon decay has lower energies, then with the steep energy spectrum of $\pi$ 's at decay, the ratio becomes larger. The interaction model also causes an uncertainty in the ratio $\left(\nu_{\mu}+\bar{\nu}_{\mu}\right) /\left(\nu_{e}+\bar{\nu}_{e}\right)$ through the $\pi$ 's spectrum at the decay. However, this is not a large effect as is seen from the right panel of Fig. 10.

We estimate the error of the ratio $\left(\nu_{\mu}+\bar{\nu}_{\mu}\right) /\left(\nu_{e}+\bar{\nu}_{e}\right)$ is $\sim \pm 2 \%$ below $100 \mathrm{GeV}$, since our atmospheric density profile is a good approximation for the one year average of the more realistic model.

\section{Uncertainty for the vertical/horizontal ratio}

In the determination of $\delta m_{23}^{2} \equiv m_{\nu_{3}}^{2}-m_{\nu_{2}}^{2}$ with atmospheric neutrino flux, the zenith angle dependence of the atmospheric neutrino flux is important. For the study of the zenith angle dependence, we calculate the quantity defined as:

$$
I_{i}^{(n)}(\cos \theta)=\int_{E 1}^{E 2} \phi_{i}\left(\cos \theta, E_{\nu}\right) E_{\nu}^{n} d E_{\nu},
$$

for $i=\nu_{\mu}, \bar{\nu}_{\mu}$, following HKKM04. The quantity $I_{\nu_{\mu}}^{(2)}$ and $I_{\bar{\nu}_{\mu}}^{(2)}$ are roughly proportional to the rate of the muon events induced by $\nu_{\mu}$ and $\bar{\nu}_{\mu}$ in the energy range from $E_{1}$ to $E_{2}$. The neutrino cross section is roughly proportional to the neutrino energy and the muon range, therefore, the target volume is proportional to the muon energy, as far as the energy loss due to the pair creations is smaller than the ionization energy loss $(\sim 500 \mathrm{GeV})$. With $E_{1}=10 \mathrm{GeV}$ and $E_{2}=1 \mathrm{TeV}$ for the integration, we calculated $I_{V}^{(2)}$ as the average of $I_{\nu_{\mu}}^{(2)}+I_{\bar{\nu}_{\mu}}^{(2)}$ over $\cos \theta_{z}=0.9-1.0$, and $I_{H}^{(2)}$ as the average over $\cos \theta_{z}=0.0-0.1$. These quantities are calculated in all the modified calculation schemes studied in Sec. III and in the original ones. They are summarized in Table I.

The stability of the zenith angle dependence of atmospheric neutrino flux may be studied with the stability of the $I_{H}^{(2)} / I_{V}^{(2)}$ ratio. We find $I_{H}^{(2)}$ and $I_{V}^{(2)}$ calculated with the modified DPMJET-III show increases from those calculated in HKKM04 by about 10\%. Therefore, the expectation value for the neutrino induced muon event will be also increased by $10 \%$. However the $I_{H}^{(2)} / I_{V}^{(2)}$ ratio is almost the same as the HKKM04 calculation. The situations are similar for other interaction models, and there are $\sim 3 \%$ variations in the $I_{H}^{(2)} / I_{V}^{(2)}$ ratios among the different calculations. Note, the different atmospheric density profile also gives $\mathrm{a} \sim \pm 0.8 \%$ variation. Those variations remain as the uncertainty for the $I_{H}^{(2)} / I_{V}^{(2)}$ ratio, or 
the uncertainty of the zenith angle dependence of the atmospheric neutrino flux.

TABLE I: Quantities calculated by Eq. 11 with $E_{1}=10 \mathrm{GeV}, E_{2}=1 \mathrm{TeV}$, and $\mathrm{n}=2 . I_{V}^{(2)}$ is the sum of $i=\nu_{\mu}$ and $\bar{\nu}_{\mu}$ for the vertical directions $\left(\cos \theta_{z}=0.9-1\right.$.), and $I_{H}^{(2)}$ for the horizontal directions $\left(\cos \theta_{z}=0 .-0.1\right)$, where $\theta_{z}$ is the zenith angle. Note, the original DPMJET-III is used in the HKKM04 calculation.

\begin{tabular}{|c|c|c|c|}
\hline \hline Calculation & $I_{V}^{(2)}\left(\mathrm{m}^{-2} \mathrm{sec}^{-1} \mathrm{GeV}^{2}\right)$ & $I_{H}^{(2)}\left(\mathrm{m}^{-2} \mathrm{sec}^{-1} \mathrm{GeV}^{2}\right)$ & $I_{H}^{(2)} / I_{V}^{(2)}$ \\
\hline \hline HKKM04 & 1634. & 3775. & 2.310 \\
HKKM04 $(\varepsilon=+0.05)$ & 1645. & 3881. & 2.285 \\
HKKM04 $(\varepsilon=-0.05)$ & 1577. & 3782. & 2.322 \\
modified DPMJET-III & 1798. & 4186. & 2.328 \\
modified Flux model & 1835. & 4295. & 2.340 \\
FLUKA'97 & 1689. & 4068. & 2.409 \\
modified FLUKA & 1725. & 4151. & 2.407 \\
Fritiof 7.02 & 1812. & 4133. & 2.281 \\
modified Fritiof & 1826. & 4183. & 2.290 \\
\hline \hline
\end{tabular}

\section{SUMMARY}

We have revised the calculation of atmospheric neutrino flux in HKKM04 with the "modified DPMJET-III" constructed in Paper I. Before the calculation, we have studied the error caused by the use of the "virtual detectors" generally far larger than the actual neutrino detectors. Such a virtual detector introduces an error, since it averages the neutrino flux over positions where the geomagnetic conditions are different from the position of target detector. At the low energy end of our calculation $(0.1 \mathrm{GeV})$, the error reaches around $5 \%$ with the virtual detector used in HKKM04 (a circle with radius $\sim 1000 \mathrm{~km}$ ), but it could be corrected comparing the average flux values of virtual detectors with different sizes. With this correction the error could be reduced to $\lesssim 1 \%$ which is much smaller than the uncertainties in other components for the calculation of atmospheric neutrino flux. In the calculation, we updated the geomagnetic field model from epoch 2000 to 2005. 
Next, we studied the robustness of the modification procedure explained in Paper I. We modified the interaction models of FLUKA'97 and Fritiof 7.02, so that they reproduce the atmospheric muon flux data (i.e., the same as the modified DPMJET-III), and calculated the atmospheric neutrino flux in the 1-dimensional scheme for the energies above $3 \mathrm{GeV}$. The neutrino fluxes calculated with those modified interaction models agree within $\pm 5 \%$ with the flux calculated with the modified DPMJET-III below $100 \mathrm{GeV}$. However, the variation increases to around $\pm 15 \%$ at $1 \mathrm{TeV}$. This is considered to be due to the differences of the $K$ productions in the modified interaction model. As the $K$ productions in the original interaction models are different, there remain the differences of the $K$ production in the modified interaction models. With this variation of the neutrino fluxes with the modified interaction models, we estimated the uncertainty of the atmospheric neutrino flux due to the uncertainty of the $K$ production in the interaction model, when they are modified to reproduce the observed muon flux correctly.

We summarized the uncertainties in the calculation of atmospheric neutrino flux including that of $K$ production in the interaction model. The relation $\Delta \phi_{\mu} / \phi_{\mu} \simeq \Delta \phi_{\nu_{\mu}} / \phi_{\nu_{\mu}} \simeq$ $\Delta \phi_{\nu_{e}} / \phi_{\nu_{e}}$, for the atmospheric lepton fluxes from the $\pi$ decay derived in Paper I is useful in this study. As we reproduce the atmospheric muon flux with a good accuracy from $1 \mathrm{GeV}$ to $1 \mathrm{TeV}$, the uncertainty of atmospheric neutrino flux due to the uncertainty of the $\pi$ productions in the interaction model is estimated from the experimental error and residual of the reconstruction of the atmospheric muon flux data. Summarizing the uncertainties of atmospheric density profile, interaction cross section and the $K$-productions in the interaction model, we estimate the uncertainty of the atmospheric neutrino flux calculated in this paper is $\sim 7 \%$ from 1 to $10 \mathrm{GeV}, \sim 14 \%$ at $100 \mathrm{GeV}$, and $\sim 25 \%$ at $1 \mathrm{TeV}$. Note the statistical error in the Monte Carlo study and uncertainty due to the calculation scheme is much smaller than those of other sources of the uncertainties. It is difficult to estimate the error at energies above $1 \mathrm{TeV}$, since the uncertainties of the primary flux and the interaction model would be larger at the corresponding energies $(\gtrsim 10 \mathrm{TeV})$ of the primary cosmic rays. Also accurately measured muon flux data are not available at the corresponding muon momenta $(\gtrsim 3 \mathrm{TeV})$ to calibrate the uncertainties.

The neutrino flux ratios are also compared among the different calculations to study the variation. Although the variations for $\nu_{\mu} / \bar{\nu}_{\mu}$ and $\nu_{e} / \bar{\nu}_{e}$ are large, the $\left(\nu_{\mu}+\bar{\nu}_{\mu}\right) /\left(\nu_{e}+\bar{\nu}_{e}\right)$ ratio is very stable $(\lesssim 2 \%)$ over the range $0.1-100 \mathrm{GeV}$. The variation of the atmospheric density 
profile gives the largest variation in the $\left(\nu_{\mu}+\bar{\nu}_{\mu}\right) /\left(\nu_{e}+\bar{\nu}_{e}\right)$ ratio. However, for an one year average, the uncertainty of this ratio will be sufficiently small.

The stability of the zenith angle dependence is also important in the study of neutrino oscillations using the atmospheric neutrino flux. We have studied the ratio [horizontal flux]/[vertical flux] ratio in the different calculations, then estimated the uncertainty. The variation or the uncertainty of the ratio is $\sim 3 \%$, and the variation of $K$-production is considered to be the main source of this uncertainty.

\section{Acknowledgments}

We greatly appreciate the contributions of J. Nishimura and A. Okada to this paper. We are grateful to P.G. Edwards for discussions and comments. We also thank the ICRR of the University of Tokyo, especially for the use of the computer system. This study was supported by Grants-in-Aid, KAKENHI(12047206), from the Ministry of Education, Culture, Sport, Science and Technology (MEXT) in Japan.

[1] T. Sanuki et al., Study of cosmic ray interaction model based on atmospheric muons for the neutrino flux calculation (2006), astro-ph/0611201.

[2] M. Honda, T. Kajita, K. Kasahara, and S. Midorikawa, Phys. Rev. D 70, 043008 (2004).

[3] S. Roesler, R. Engel, and J. Ranft, The Monte Carlo event generator DPMJET-III (2000), hep-ph/0012252.

[4] S. Haino et al. (BESS), Phys. Lett. B594, 35 (2004).

[5] T. Sanuki et al., Phys. Lett. B541, 234 (2002), see also erratum [28].

[6] P. Achard et al. (L3), Phys. Lett. B598, 15 (2004).

[7] See http://www.ngdc.noaa.gov/IAGA/vmod/igrf.html.

[8] G. D. Barr, T. K. Gaisser, P. Lipari, S. Robbins, and T. Stanev, Phys. Rev. D70, 023006 (2004).

[9] A. Fasso, A. Ferrari, and P. Sala (2001), prepared for International Conference on Advanced Monte Carlo for Radiation Physics, Particle Transport Simulation and Applications (MC 2000), Lisbon, Portugal, 23-26 Oct 2000. 
[10] H. Pi, Comput. Phys. Commun. 71, 173 (1992).

[11] G. D. Barr, T. K. Gaisser, S. Robbins, and T. Stanev, Phys. Rev. D74, 094009 (2006).

[12] J. Alcaraz et al. (AMS), Phys. Lett. B490, 27 (2000).

[13] J. Alcaraz et al. (AMS), Phys. Lett. B494, 193 (2000).

[14] T. Sanuki et al. (BESS), Astrophys. J. 545, 1135 (2000).

[15] T. K. Gaisser and M. Honda, Ann. Rev. Nucl. Part. Sci. 52, 153 (2002).

[16] T. K. Gaisser et al., Proc. of the 27th Int. Cosmic Ray Conf., Hamburg 5, 1643 (2001).

[17] See http://nssdc.gsfc.nasa.gov/space/model/atmos/us_standard.html.

[18] M. Honda, T. Kajita, K. Kasahara, and S. Midorikawa, Phys. Rev. D64, 053011 (2001).

[19] G. Battistoni, A. Ferrari, T. Montaruli, and P. R. Sala, Astropart. Phys. 19, 269 (2003).

[20] G. Battistoni, A. Ferrari, T. Montaruli, and P. R. Sala (2003), hep-ph/0305208.

[21] J. Wentz et al., Phys. Rev. D67, 073020 (2003).

[22] J. Favier, R. Kossakowski, and J. P. Vialle, Phys. Rev. D68, 093006 (2003).

[23] Y. Liu, L. Derome, and M. Buenerd, Phys. Rev. D67, 073022 (2003).

[24] V. Agrawal, T. K. Gaisser, P. Lipari, and T. Stanev, Phys. Rev. D53, 1314 (1996).

[25] K. Abe et al., Proc. of the 28th Int. Cosmic Ray Conf., Tsukuba 3, 1463 (2003), see also astro-ph/0312632.

[26] J. P. Wefel et al. (ATIC-2), Proc. of the 29th Int. Cosmic Ray Conf., Pune 3, 105 (2005).

[27] K. Abe et al. (BESS), Phys. Lett. B564, 8 (2003).

[28] T. Sanuki et al., Phys. Lett. B581, 272 (2004). 


\section{APPENDIX A: ATMOSPHERIC NEUTRINO FLUX BELOW 10 GEV}

Here we tabulate the calculated low energy $(0.1-10 \mathrm{GeV})$ atmospheric neutrino flux for 3 locations - Kamioka, Sudbury (North America), and Gran Sasso - averaging them in the zenith angle bins with $\Delta \cos \theta_{z}=0.1$, where $\theta_{z}$ is the zenith angle of the arrival direction of the neutrino, in Tables II-XX1.

The atmospheric neutrino flux for solar minimum at sea level could be calculated as

$$
\phi_{\nu}=[\text { Value in table }] \times[\text { Norm }]\left(\mathrm{m}^{-2} \mathrm{sec}^{-1} \mathrm{sr}^{-1} \mathrm{GeV}^{-1}\right)
$$

for each neutrino energy, kind of neutrino, observation site, and zenith angle bin.

We have also calculated the flux of atmospheric neutrinos for Soudan2 and Frejus sites. The flux at Soudan2 site is almost identical to that of the SNO site (Sudbury). The flux at the Frejus site is a little higher $(3 \%$ at $1 \mathrm{GeV}$ and $10 \%$ at $0.1 \mathrm{GeV})$ than that for Gran Sasso. However, the flux for Gran Sasso could be used as the approximate flux for the Frejus site. Therefore, we select the fluxes for Kamioka, Sudbury, and Gran Sasso, to save space in this paper.

The difference of the fluxes at different sites is due to the rigidity cutoff of the cosmic rays. Note, the difference of the terrain above the neutrino detector is not considered for each observation site in these tables. It may be important for the neutrino detectors constructed under high mountains.

More detailed flux tables will be available from the web site: http://www.icrr.u-tokyo.ac.jp/ mhonda, from $0.1 \mathrm{GeV}$ to $10 \mathrm{TeV}$, for solar maximum and solar minimum, with and without the consideration of the terrain, and for Kamioka, Sudbury, Soudan2, Gran Sasso, and Frejus sites. Dividing the azimuthal angels into 12 bins, the flux table for each azimuthal bin will also be available for each calculation conditions stated above. 
TABLE II: Neutrino flux $\left(\mathrm{m}^{-2} \mathrm{sec}^{-1} \mathrm{sr}^{-1} \mathrm{GeV}^{-1}\right)$ for $1.0 \geq \cos \theta_{z}>0.9$

\begin{tabular}{|c|c|c|c|c|c|c|c|c|c|c|c|c|c|}
\hline & & Kam & aioka & & & Sud & lbury & & & Gran & Sasso & & \\
\hline$E_{\nu}(\mathrm{GeV})$ & $\nu_{\mu}$ & $\bar{\nu}_{\mu}$ & $\nu_{e}$ & $\bar{\nu}_{e}$ & $\nu_{\mu}$ & $\bar{\nu}_{\mu}$ & $\nu_{e}$ & $\bar{\nu}_{e}$ & $\nu_{\mu}$ & $\bar{\nu}_{\mu}$ & $\nu_{e}$ & $\bar{\nu}_{e}$ & Norm \\
\hline .1000 & 35.46 & 35.39 & 17.88 & 17.16 & 90.72 & 90.33 & 49.96 & 37.77 & 59.58 & 59.59 & 30.83 & 27.54 & $10^{2}$ \\
\hline .1259 & 29.84 & 29.77 & 14.82 & 14.08 & 74.31 & 73.99 & 40.25 & 30.39 & 49.52 & 49.54 & 25.23 & 22.43 & $10^{2}$ \\
\hline .1585 & 24.52 & 24.39 & 11.84 & 11.17 & 59.41 & 58.77 & 31.11 & 23.53 & 40.32 & 40.14 & 19.95 & 17.56 & $10^{2}$ \\
\hline .1995 & 19.37 & 19.19 & 9.13 & 8.54 & 44.88 & 44.37 & 22.99 & 17.44 & 31.40 & 31.14 & 15.17 & 13.24 & $10^{2}$ \\
\hline .2512 & 14.49 & 14.32 & 6.78 & 6.25 & 31.37 & 31.27 & 16.14 & 12.30 & 22.95 & 22.77 & 11.02 & 9.57 & $10^{2}$ \\
\hline .3162 & 10.34 & 10.16 & 4.81 & 4.40 & 20.54 & 20.53 & 10.66 & 8.20 & 15.88 & 15.69 & 7.62 & 6.56 & $10^{2}$ \\
\hline .3981 & 7.11 & 6.93 & 3.27 & 2.96 & 12.83 & 12.81 & 6.64 & 5.16 & 10.50 & 10.31 & 5.04 & 4.28 & $10^{2}$ \\
\hline .5012 & 47.36 & 45.76 & 21.41 & 19.05 & 77.62 & 77.08 & 39.43 & 30.93 & 66.99 & 65.30 & 31.84 & 26.68 & $10^{1}$ \\
\hline .6310 & 30.74 & 29.25 & 13.56 & 11.87 & 45.74 & 44.82 & 22.58 & 17.79 & 41.53 & 39.84 & 19.21 & 15.98 & $10^{1}$ \\
\hline .7943 & 19.36 & 18.19 & 8.32 & 7.14 & 26.34 & 25.39 & 12.57 & 9.93 & 24.80 & 23.52 & 11.25 & 9.20 & $10^{1}$ \\
\hline 1.000 & 11.83 & 10.99 & 4.95 & 4.15 & 14.86 & 14.08 & 6.83 & 5.39 & 14.31 & 13.47 & 6.39 & 5.12 & $10^{1}$ \\
\hline 1.259 & 7.07 & 6.44 & 2.84 & 2.33 & 8.25 & 7.66 & 3.61 & 2.82 & 8.10 & 7.50 & 3.46 & 2.76 & $10^{1}$ \\
\hline 1.585 & 41.20 & 36.72 & 15.69 & 12.68 & 45.34 & 41.01 & 18.63 & 14.40 & 45.03 & 40.54 & 18.16 & 14.23 & 1 \\
\hline 1.995 & 23.40 & 20.33 & 8.37 & 6.63 & 24.71 & 21.64 & 9.39 & 7.21 & 24.61 & 21.46 & 9.26 & 7.07 & 1 \\
\hline 2.512 & 12.91 & 10.89 & 4.30 & 3.34 & 13.31 & 11.27 & 4.60 & 3.50 & 13.29 & 11.23 & 4.57 & 3.47 & 1 \\
\hline 3.162 & 69.77 & 57.10 & 21.3 & 16.36 & 70.95 & 58.27 & 22.11 & 16.69 & 70.90 & 58.10 & 22.10 & 16.68 & $10^{-1}$ \\
\hline 3.981 & 37.04 & 29.54 & 10.21 & 7.80 & 37.31 & 29.85 & 10.43 & 7.84 & 37.25 & 29.67 & 10.44 & 7.80 & $10^{-1}$ \\
\hline 5.012 & 19.31 & 15.08 & 4.72 & 3.61 & 19.30 & 15.07 & 4.78 & 3.60 & 19.28 & 15.02 & 4.76 & 3.58 & $10^{-1}$ \\
\hline 6.310 & 99.07 & 76.11 & 21.53 & 16.35 & 98.85 & 75.86 & 21.61 & 16.28 & 98.85 & 75.81 & 21.51 & 16.26 & $10^{-2}$ \\
\hline 7.943 & 50.36 & 38.19 & 9.71 & 7.31 & 50.38 & 38.23 & 9.70 & 7.32 & 50.39 & 38.24 & 9.72 & 7.33 & $10^{-2}$ \\
\hline 10.00 & 25.57 & 19.21 & 4.31 & 3.27 & 25.58 & 19.22 & 4.31 & 3.27 & 25.58 & 19.22 & 4.31 & 3.27 & $10^{-2}$ \\
\hline
\end{tabular}


TABLE III: Neutrino flux $\left(\mathrm{m}^{-2} \mathrm{sec}^{-1} \mathrm{sr}^{-1} \mathrm{GeV}^{-1}\right)$ for $0.9 \geq \cos \theta_{z}>0.8$

\begin{tabular}{|c|c|c|c|c|c|c|c|c|c|c|c|c|c|}
\hline & & Kan & ioka & & & Sudb & oury & & & Gran & Sasso & & \\
\hline$E_{\nu}(\mathrm{GeV})$ & $\nu_{\mu}$ & $\bar{\nu}_{\mu}$ & $\nu_{e}$ & $\bar{\nu}_{e}$ & $\nu_{\mu}$ & $\bar{\nu}_{\mu}$ & $\nu_{e}$ & $\bar{\nu}_{e}$ & $\nu_{\mu}$ & $\bar{\nu}_{\mu}$ & $\nu_{e}$ & $\bar{\nu}_{e}$ & Norm \\
\hline .1000 & 36.34 & 36.30 & 18.31 & 17.49 & 93.87 & 93.35 & 51.63 & 38.95 & 61.25 & 61.37 & 731.66 & 28.28 & $10^{2}$ \\
\hline .1259 & 30.34 & 30.31 & 15.18 & 14.31 & 76.32 & 75.90 & 41.46 & 31.30 & 50.69 & 50.69 & 25.96 & 22.92 & $10^{2}$ \\
\hline .1585 & 24.78 & 24.68 & 12.11 & 11.34 & 60.55 & 60.01 & 31.93 & 24.15 & 41.00 & 40.87 & 20.44 & 17.97 & $10^{2}$ \\
\hline .1995 & 19.47 & 19.31 & 9.32 & 8.68 & 45.52 & 45.07 & 23.55 & 17.88 & 31.77 & 31.59 & 15.52 & 13.58 & $10^{2}$ \\
\hline .2512 & 14.52 & 14.37 & 6.91 & 6.39 & 31.74 & 31.68 & 16.56 & 12.63 & 23.21 & 23.04 & 411.34 & 9.82 & $10^{2}$ \\
\hline .3162 & 10.36 & 10.21 & 4.92 & 4.50 & 20.80 & 20.91 & 11.00 & 8.45 & 16.04 & 15.91 & 7.88 & 6.77 & $10^{2}$ \\
\hline .3981 & 7.13 & 6.98 & 3.36 & 3.03 & 13.03 & 13.08 & 6.89 & 5.35 & 10.63 & 10.49 & 5.21 & 4.44 & $10^{2}$ \\
\hline .5012 & 47.55 & 46.14 & 22.09 & 19.61 & 79.02 & 78.66 & 41.05 & 32.29 & 68.19 & 66.52 & 33.00 & 27.78 & $10^{1}$ \\
\hline .6310 & 30.91 & 29.62 & 14.13 & 12.32 & 46.74 & 45.98 & 23.72 & 18.71 & 42.27 & 40.84 & 420.23 & 16.74 & $10^{1}$ \\
\hline .7943 & 19.53 & 18.48 & 8.73 & 7.49 & 26.96 & 26.13 & 13.36 & 10.50 & 25.30 & 24.20 & 11.91 & 9.72 & $10^{1}$ \\
\hline 1.000 & 11.99 & 11.19 & 5.19 & 4.38 & 15.21 & 14.49 & 7.31 & 5.72 & 14.68 & 13.88 & 6.75 & 5.44 & $10^{1}$ \\
\hline 1.259 & 7.15 & 6.58 & 2.99 & 2.46 & 8.43 & 7.90 & 3.86 & 3.02 & 8.33 & 7.75 & 5.72 & 2.94 & $10^{1}$ \\
\hline 1.585 & 41.62 & 37.57 & 16.74 & 13.38 & 46.43 & 42.49 & 19.96 & 15.61 & 46.43 & 42.10 & 19.80 & 15.25 & 1 \\
\hline 1.995 & 23.68 & 20.87 & 9.03 & 7.10 & 25.40 & 22.50 & 10.14 & 7.85 & 25.38 & 22.33 & 10.09 & 7.67 & 1 \\
\hline 2.512 & 13.17 & 11.27 & 4.68 & 3.64 & 13.69 & 11.73 & 5.04 & 13.83 & 13.62 & 11.69 & 4.97 & 3.80 & 1 \\
\hline 3.162 & 71.46 & 59.3 & 23.31 & 17.95 & 72.94 & 60.5 & 24.33 & 18.34 & 72.43 & 60.45 & 24.05 & 18.25 & $10^{-1}$ \\
\hline 3.981 & 37.94 & 30.64 & 11.23 & 8.55 & 38.39 & 30.99 & 11.44 & 8.66 & 38.19 & 30.88 & 11.44 & 8.54 & $10^{-1}$ \\
\hline 5.012 & 19.81 & 15.62 & 5.26 & 3.94 & 19.85 & 15.68 & 5.30 & 4.00 & 19.82 & 15.64 & $4 \quad 5.30$ & 3.95 & $10^{-1}$ \\
\hline 6.310 & 101.72 & 78.98 & 24.06 & 18.01 & 101.56 & 78.98 & 24.12 & 18.15 & 101.59 & 78.96 & 24.10 & 18.10 & $10^{-2}$ \\
\hline 7.943 & 51.69 & 39.76 & 10.83 & 8.19 & 51.72 & 39.7 & 10.81 & 8.17 & 51.72 & 39.77 & 710.82 & 8.18 & $10^{-2}$ \\
\hline 10.00 & 26.25 & 19.97 & 4.84 & 3.67 & 26.25 & 19.97 & 4.84 & 43.67 & 26.25 & 19.97 & $7 \quad 4.84$ & 3.67 & $10^{-2}$ \\
\hline
\end{tabular}


TABLE IV: Neutrino flux $\left(\mathrm{m}^{-2} \mathrm{sec}^{-1} \mathrm{sr}^{-1} \mathrm{GeV}^{-1}\right)$ for $0.8 \geq \cos \theta_{z}>0.7$

\begin{tabular}{|c|c|c|c|c|c|c|c|c|c|c|c|c|c|}
\hline & & Kam & nioka & & & Sudl & bury & & & Gran & I Sasso & & \\
\hline$E_{\nu}(\mathrm{GeV})$ & $\nu_{\mu}$ & $\bar{\nu}_{\mu}$ & $\nu_{e}$ & $\bar{\nu}_{e}$ & $\nu_{\mu}$ & $\bar{\nu}_{\mu}$ & $\nu_{e}$ & $\bar{\nu}_{e}$ & $\nu_{\mu}$ & $\bar{\nu}_{\mu}$ & $\nu_{e}$ & $\bar{\nu}_{e}$ & Norm \\
\hline .1000 & 7.43 & 37.60 & 18.95 & 17.91 & 97.85 & 97.43 & 53.65 & 40.41 & 63.47 & 63.64 & 432.73 & $\begin{array}{ll}329.17 \\
\end{array}$ & $10^{2}$ \\
\hline .1259 & 31.05 & 31.09 & 15.54 & 14.62 & 78.82 & 78.52 & 42.82 & 32.39 & 52.15 & 52.23 & 26.65 & 523.63 & $10^{2}$ \\
\hline .1585 & 25.21 & 25.12 & 12.37 & 11.55 & 61.99 & 61.52 & 32.90 & 24.88 & 41.78 & 41.77 & 21.00 & 018.48 & $10^{2}$ \\
\hline .1995 & 19.67 & 19.56 & 9.54 & 8.83 & 46.27 & 45.90 & 24.23 & 18.37 & 32.18 & 32.05 & 15.95 & 513.93 & $10^{2}$ \\
\hline .2512 & 14.62 & 14.51 & 7.07 & 6.51 & 32.14 & 32.17 & 17.03 & 12.99 & 23.48 & 23.33 & 11.61 & 10.07 & $10^{2}$ \\
\hline .3162 & 10.40 & 10.28 & 5.04 & 4.60 & 21.06 & 21.24 & 11.32 & 8.71 & 16.19 & 16.11 & 8.08 & 8.95 & $10^{2}$ \\
\hline .3981 & 7.16 & 7.03 & 3.47 & 3.12 & 13.23 & 13.32 & 7.13 & 5.54 & 10.76 & 10.65 & 5.38 & 8.58 & $10^{2}$ \\
\hline .5012 & 4.80 & 4.66 & 2.30 & 2.03 & 8.06 & 8.04 & 4.29 & 3.36 & 6.93 & 6.77 & $7 \quad 3.45$ & $5 \quad 2.89$ & $10^{2}$ \\
\hline .6310 & 31.29 & 30.04 & 14.75 & 12.84 & 47.73 & 47.17 & 24.92 & 19.65 & 42.99 & 41.72 & 21.28 & 3 17.61 & $10^{1}$ \\
\hline .7943 & 19.82 & 18.80 & 9.10 & 7.84 & 27.57 & 26.93 & 14.06 & 11.13 & 25.80 & 24.83 & 12.59 & 910.29 & $10^{1}$ \\
\hline 1.000 & 12.18 & 11.42 & 5.46 & 4.62 & 15.59 & 15.00 & 7.74 & 6.10 & 15.04 & 14.32 & 7.19 & 5.77 & $10^{1}$ \\
\hline 1.259 & 7.27 & 6.73 & 3.20 & 2.63 & 8.69 & 8.22 & 4.16 & 3.25 & 8.53 & 8.04 & 4.01 & 13.15 & $10^{1}$ \\
\hline 1.585 & 42.47 & 38.63 & 18.03 & 14.52 & 47.94 & 44.26 & 21.79 & 16.78 & 47.55 & 43.82 & 21.47 & 716.56 & 1 \\
\hline 1.995 & 24.29 & 21.57 & 9.74 & 7.74 & 26.19 & 23.50 & 11.13 & 8.46 & 26.12 & 23 & 10.97 & $7 \quad 8.39$ & 1 \\
\hline 2.512 & 13.48 & 11.71 & 5.09 & 3.97 & 14.13 & 12.31 & 5.53 & 4.20 & 14.05 & 12.21 & 5.46 & 64.16 & 1 \\
\hline 3.162 & 73.24 & 61.82 & 25.58 & 19.67 & 75.34 & 63.62 & 26.8 & 20.41 & 74.89 & 63 & 26.69 & 20.17 & $10^{-1}$ \\
\hline 3.981 & 39.02 & 31.97 & 12.40 & 9.47 & 39.65 & 32.43 & 12.76 & 9.66 & 39.57 & 32.43 & 12.78 & 9.57 & $10^{-1}$ \\
\hline 5.012 & 20.31 & 16.33 & 5.86 & 4.43 & 20.51 & 16.38 & 5.94 & 4.46 & 20.50 & 16.42 & 5.94 & 4.47 & $10^{-1}$ \\
\hline 6.310 & 10.43 & 8.25 & 2.71 & 2.04 & 10.47 & 8.25 & 2.71 & 2.04 & 10.47 & 8.26 & 2.71 & 2.04 & $10^{-}$ \\
\hline 7.943 & 53.20 & 41.48 & 12.25 & 9.24 & 53.15 & 41.5 & 12.24 & 9.24 & 53.15 & 41.49 & 12.25 & 9.23 & $10^{-2}$ \\
\hline 10.00 & 27.03 & 20.85 & 5.48 & 4.16 & 27.02 & 20.85 & 5.48 & 4.16 & 27.02 & 20.85 & 5.48 & 34.15 & $10^{-2}$ \\
\hline
\end{tabular}


TABLE V: Neutrino flux $\left(\mathrm{m}^{-2} \mathrm{sec}^{-1} \mathrm{sr}^{-1} \mathrm{GeV}^{-1}\right)$ for $0.7 \geq \cos \theta_{z}>0.6$

\begin{tabular}{|c|c|c|c|c|c|c|c|c|c|c|c|c|c|}
\hline \multirow[b]{2}{*}{$E_{\nu}(\mathrm{GeV})$} & \multicolumn{4}{|c|}{ Kamioka } & \multicolumn{4}{|c|}{ Sudbury } & \multicolumn{4}{|c|}{ Gran Sasso } & \multirow[b]{2}{*}{ Norm } \\
\hline & $\nu_{\mu}$ & $\bar{\nu}_{\mu}$ & $\nu_{e}$ & $\bar{\nu}_{e}$ & $\nu_{\mu}$ & $\bar{\nu}_{\mu}$ & $\nu_{e}$ & $\bar{\nu}_{e}$ & $\nu_{\mu}$ & $\bar{\nu}_{\mu}$ & $\nu_{e}$ & $\bar{\nu}_{e}$ & \\
\hline .1000 & 38.96 & 39.24 & 19.66 & 18.57 & 103.24 & 102.73 & 56.39 & 42.58 & 66.52 & 66.76 & 34.19 & 30.51 & $10^{2}$ \\
\hline .1259 & 31.96 & 32.10 & 16.10 & 15.08 & 82.28 & 81.96 & 44.72 & 33.79 & 54.06 & 54.26 & 27.68 & 24.51 & $10^{2}$ \\
\hline .1585 & 25.67 & 25.72 & 12.73 & 11.87 & 63.95 & 63.55 & 34.10 & 25.82 & 42.93 & 42.97 & 21.66 & 18.99 & $10^{2}$ \\
\hline .1995 & 19.93 & 19.86 & 9.76 & 9.03 & 47.25 & 47.00 & 24.98 & 18.99 & 32.79 & 32.68 & 16.36 & 14.26 & $10^{2}$ \\
\hline .2512 & 14.78 & 14.66 & 7.24 & 6.62 & 32.69 & 32.71 & 17.50 & 13.40 & 23.75 & 23.63 & 11.92 & 10.33 & $10^{2}$ \\
\hline .3162 & 10.48 & 10.37 & 5.18 & 4.70 & 21.38 & 21.57 & 11.65 & 8.98 & 16.43 & 16.30 & 8.31 & 7.14 & $10^{2}$ \\
\hline .3981 & 7.21 & 7.10 & 3.56 & 3.20 & 13.44 & 13.56 & 7.38 & 5.74 & 10.92 & 10.80 & 5.55 & 4.72 & $10^{2}$ \\
\hline .5012 & 4.84 & 4.73 & 2.37 & 2.10 & 8.20 & 8.21 & 4.46 & 3.51 & 7.02 & 6.91 & 3.56 & 3.00 & $10^{2}$ \\
\hline .6310 & 31.56 & 30.54 & 15.33 & 13.35 & 48.68 & 48.33 & 26.13 & 20.63 & 43.75 & 42.65 & 22.10 & 18.33 & $10^{1}$ \\
\hline .7943 & 20.05 & 19.20 & 9.60 & 8.25 & 28.23 & 27.74 & 14.88 & 11.76 & 26.35 & 25.48 & 13.24 & 10.81 & $10^{1}$ \\
\hline 1.000 & 12.40 & 11.74 & 5.82 & 4.92 & 16.04 & 15.55 & 8.28 & 6.50 & 15.38 & 14.76 & 7.65 & 6.17 & $10^{1}$ \\
\hline 1.259 & 7.44 & 6.94 & 3.41 & 2.81 & 8.94 & 8.56 & 4.50 & 3.47 & 8.79 & 8.28 & 4.26 & 3.39 & $10^{1}$ \\
\hline 1.585 & 43.64 & 40.00 & 19.29 & 15.55 & 49.31 & 46.40 & 23.67 & 18.18 & 49.07 & 45.41 & 22.95 & 17.97 & 1 \\
\hline 1.995 & 24.99 & 22.42 & 10.54 & 8.39 & 26.95 & 24.69 & 12.11 & 9.31 & 26.85 & 24.40 & 11.98 & 9.24 & 1 \\
\hline 2.512 & 13.87 & 12.17 & 5.57 & 4.35 & 14.55 & 12.89 & 6.10 & 4.62 & 14.53 & 12.80 & 6.02 & 4.61 & 1 \\
\hline 3.162 & 7.53 & 6.45 & 2.83 & 2.17 & 7.78 & 6.66 & 3.01 & 2.26 & 7.76 & 6.64 & 2.97 & 2.25 & 1 \\
\hline 3.981 & 40.15 & 33.58 & 13.78 & 10.50 & 40.97 & 34.20 & 14.42 & 10.89 & 40.86 & 34.13 & 14.31 & 10.78 & $10^{-1}$ \\
\hline 5.012 & 20.89 & 17.17 & 6.57 & 5.00 & 21.05 & 17.33 & 6.72 & 5.07 & 21.13 & 17.31 & 6.68 & 5.06 & $10^{-1}$ \\
\hline 6.310 & 10.75 & 8.68 & 3.07 & 2.32 & 10.76 & 8.71 & 3.08 & 2.32 & 10.81 & 8.70 & 3.07 & 2.32 & $10^{-1}$ \\
\hline 7.943 & 55.08 & 43.69 & 14.04 & 10.55 & 55.08 & 43.64 & 14.02 & 10.56 & 55.01 & 43.65 & 14.04 & 10.55 & $10^{-2}$ \\
\hline 10.00 & 27.99 & 21.96 & 6.34 & 4.78 & 28.00 & 21.96 & 6.33 & 4.79 & 27.98 & 21.96 & 6.34 & 4.78 & $10^{-2}$ \\
\hline
\end{tabular}


TABLE VI: Neutrino flux $\left(\mathrm{m}^{-2} \mathrm{sec}^{-1} \mathrm{sr}^{-1} \mathrm{GeV}^{-1}\right)$ for $0.6 \geq \cos \theta_{z}>0.5$

\begin{tabular}{|c|c|c|c|c|c|c|c|c|c|c|c|c|c|}
\hline & & Kam & nioka & & & Sudbr & ury & & & Gran & Sasso & & \\
\hline$E_{\nu}(\mathrm{GeV})$ & $\nu_{\mu}$ & $\bar{\nu}_{\mu}$ & $\nu_{e}$ & $\bar{\nu}_{e}$ & $\nu_{\mu}$ & $\bar{\nu}_{\mu}$ & $\nu_{e}$ & $\bar{\nu}_{e}$ & $\nu_{\mu}$ & $\bar{\nu}_{\mu}$ & $\nu_{e}$ & $\bar{\nu}_{e}$ & Norm \\
\hline 1000 & 41.34 & 41.60 & 20.84 & 19.58 & 110.93 & 110.41 & 60.17 & 45.48 & 71.14 & 71.28 & 36.16 & 632.23 & $10^{2}$ \\
\hline .1259 & 33.50 & 33.71 & 16.84 & 15.76 & 87.17 & 86.97 & 47.31 & 35.73 & 57.05 & 57.20 & 29.06 & 625.74 & $10^{2}$ \\
\hline .1585 & 26.58 & 26.65 & 13.22 & 12.24 & 66.69 & 66.36 & 35.75 & 27.06 & 44.66 & 44.62 & 22.52 & 219.82 & $10^{2}$ \\
\hline .1995 & 20.36 & 20.36 & 10.07 & 9.25 & 48.62 & 48.44 & 25.98 & 19.76 & 33.59 & 33.53 & 16.91 & 114.76 & $10^{2}$ \\
\hline .2512 & 14.93 & 14.90 & 7.45 & 6.80 & 33.30 & 33.48 & 18.11 & 13.83 & 24.10 & 24.08 & 12.26 & 10.60 & $10^{2}$ \\
\hline .3162 & 10.58 & 10.53 & 5.33 & 4.80 & 21.69 & 21.96 & 12.02 & 9.25 & 16.59 & 16.54 & 8.54 & $4 \quad 7.33$ & $10^{2}$ \\
\hline .3981 & 7.28 & 7.21 & 3.68 & 3.27 & 13.63 & 13.80 & 7.61 & 5.93 & 11.03 & 10.94 & 5.72 & 24.86 & $10^{2}$ \\
\hline .5012 & 4.89 & 4.79 & 2.46 & 2.16 & 8.34 & 8.39 & 4.63 & 3.65 & 7.11 & 7.00 & 3.69 & 3.10 & $10^{2}$ \\
\hline .6310 & 32.03 & 31.03 & 15.93 & 13.85 & 49.80 & 49.64 & 27.40 & 21.64 & 44.49 & 43.45 & 23.14 & 419.11 & $10^{1}$ \\
\hline .7943 & 20.41 & 19.58 & 10.05 & 8.61 & 28.99 & 28.61 & 15.74 & 12.44 & 26.92 & 26.12 & 13.96 & 11.37 & $10^{1}$ \\
\hline 1.000 & 12.62 & 12.01 & 6.16 & 5.18 & 16.50 & 16.11 & 8.80 & 6.94 & 15.77 & 15.23 & 8.11 & 6.55 & $10^{1}$ \\
\hline 1.259 & 7.59 & 7.15 & 3.66 & 3.00 & 9.25 & 8.90 & 4.82 & 3.76 & 9.04 & 8.62 & 4.57 & 73.66 & $10^{1}$ \\
\hline 1.585 & 44.53 & 41.53 & 20.94 & 16.95 & 51.08 & 48.40 & 25.77 & 19.85 & 50.78 & 47.52 & 24.97 & 719.64 & 1 \\
\hline 1.995 & 25.55 & 23.43 & 11.51 & 9.26 & 27.92 & 25.86 & 13.42 & 10.24 & 27.96 & 25.57 & 13.19 & 10.13 & 1 \\
\hline 2.512 & 14.29 & 12.76 & 6.14 & 4.82 & 15.16 & 13.59 & 6.80 & 5.16 & 15.06 & 13.48 & 6.70 & 5.11 & 1 \\
\hline 3.162 & 7.78 & 6.78 & 3.15 & 2.43 & 8.12 & 7.07 & 3.37 & 2.53 & 8.04 & 7.03 & 3.33 & 32.53 & 1 \\
\hline 3.981 & 41.43 & 35.37 & 15.62 & 11.92 & 42.69 & 36.36 & 16.34 & 12.14 & 42.51 & 36.19 & 16.27 & 12.26 & $10^{-1}$ \\
\hline 5.012 & 21.68 & 18.17 & 7.55 & 5.70 & 22.03 & 18.38 & 7.70 & 5.76 & 22.01 & 18.34 & 7.67 & 5.80 & $10^{-1}$ \\
\hline 6.310 & 11.19 & 9.20 & 3.55 & 2.66 & 11.24 & 9.22 & 3.56 & 2.68 & 11.24 & 9.21 & 3.55 & 2.68 & $10^{-1}$ \\
\hline 7.943 & 57.18 & 46.27 & 16.2 & 12.26 & 57.12 & 46.26 & 16.27 & 12.24 & 57.13 & 46.2 & 16.29 & 12.24 & $10^{-2}$ \\
\hline 10.00 & 29.11 & 23.29 & 7.42 & 5.60 & 29.10 & 23.28 & 7.42 & 5.60 & 29.10 & 23.29 & 7.42 & 5.60 & $10^{-2}$ \\
\hline
\end{tabular}


TABLE VII: Neutrino flux $\left(\mathrm{m}^{-2} \mathrm{sec}^{-1} \mathrm{sr}^{-1} \mathrm{GeV}^{-1}\right)$ for $0.5 \geq \cos \theta_{z}>0.4$

\begin{tabular}{|c|c|c|c|c|c|c|c|c|c|c|c|c|c|}
\hline & & Kar & aioka & & & Sudl & bury & & & Gran & Sasso & & \\
\hline$E_{\nu}(\mathrm{GeV})$ & $\nu_{\mu}$ & $\bar{\nu}_{\mu}$ & $\nu_{e}$ & $\bar{\nu}_{e}$ & $\nu_{\mu}$ & $\bar{\nu}_{\mu}$ & $\nu_{e}$ & $\bar{\nu}_{e}$ & $\nu_{\mu}$ & $\bar{\nu}_{\mu}$ & $\nu_{e}$ & $\bar{\nu}_{e}$ & Norm \\
\hline .1000 & 4.49 & 4.54 & 2.25 & 2.11 & 12.26 & 12.16 & 6.60 & 4.98 & 7.78 & 7.79 & 3.94 & $=3.51$ & $10^{3}$ \\
\hline .1259 & 35.90 & 36.26 & 18.03 & 16.69 & 94.88 & 94.37 & 51.25 & 38.64 & 61.36 & 61.44 & 31.15 & 27.57 & $10^{2}$ \\
\hline .1585 & 27.97 & 28.19 & 13.99 & 12.88 & 71.11 & 70.76 & 38.20 & 28.89 & 47.21 & 47.25 & 23.91 & 20.97 & $10^{2}$ \\
\hline .1995 & 21.10 & 21.15 & 10.56 & 9.64 & 50.74 & 50.71 & 27.39 & 20.82 & 34.96 & 34.95 & 17.73 & 15.46 & $10^{2}$ \\
\hline .2512 & 5.30 & 15.29 & 7.75 & 6.98 & 34.28 & 34.52 & 18.88 & 14.45 & 24.71 & 24.70 & 12.68 & 11.01 & $10^{2}$ \\
\hline .3162 & 10.76 & 10.71 & 5.50 & 4.91 & 22.20 & 22.47 & 12.44 & 9.63 & 16.87 & 16.83 & 8.78 & 7.56 & $10^{2}$ \\
\hline .3981 & 7.36 & 7.30 & 3.78 & 3.34 & 13.91 & 14.08 & 7.87 & 6.16 & 11.16 & 11.08 & 5.88 & 5.00 & $10^{2}$ \\
\hline .5012 & 4.92 & 4.85 & 2.53 & 2.21 & 8.49 & 8.56 & 4.81 & 3.79 & 7.18 & 7.08 & 3.80 & 3.20 & $10^{2}$ \\
\hline .6310 & 32.22 & 31.44 & 16.53 & 14.33 & 50.83 & 50.88 & 28.56 & 22.70 & 44.96 & 44.11 & 23.84 & 19.89 & $10^{1}$ \\
\hline .7943 & 20.58 & 19.88 & 10.54 & 8.98 & 29.70 & 29.52 & 16.59 & 13.15 & 27.31 & 26.70 & 14.53 & 11.95 & $10^{1}$ \\
\hline 1.000 & 12.77 & 12.24 & 6.53 & 5.43 & 16.97 & 16.73 & 9.42 & 7.39 & 16.11 & 15.67 & 8.59 & 6.94 & $10^{1}$ \\
\hline 1.259 & 7.71 & 7.33 & 3.90 & 3.19 & 9.57 & 9.30 & 5.21 & 4.07 & 9.28 & 8.93 & 4.92 & 3.90 & $10^{1}$ \\
\hline 1.585 & 45.46 & 42.70 & 22.52 & 18.15 & 53.19 & 50.71 & 28.14 & 21.85 & 52.39 & 49 & 27.16 & 21.21 & 1 \\
\hline 1.9 & 26.2 & 24.22 & 12.59 & 9.99 & 29.17 & 27.22 & 14.84 & 11.41 & 28.95 & 26. & 14.45 & 11.15 & 1 \\
\hline 2.512 & 14.76 & 13.35 & 6.82 & 5.33 & 15.83 & 14.41 & 7.58 & 5.77 & 15.63 & 14.24 & 7.43 & 5.69 & 1 \\
\hline 3.162 & 8.08 & 7.16 & 3.54 & 2.74 & 8.45 & 7.55 & 3.80 & 2.86 & 8.36 & 7.44 & 3.75 & 2.84 & 1 \\
\hline 3.981 & 43.16 & 37.49 & 17.75 & 13.58 & 44.41 & 38.93 & 18.69 & 14.00 & 44.23 & 38.65 & 18.58 & 13.92 & $10^{-1}$ \\
\hline 5.012 & 22.67 & 19.28 & 8.69 & 6.59 & 23.03 & 19.70 & 8.89 & 6.70 & 22.95 & 19.69 & 8.91 & 6.68 & $10^{-1}$ \\
\hline 6.310 & 11.72 & 9.82 & 4.14 & 3.12 & 11.78 & 9.88 & 4.15 & 3.13 & 11.75 & 9.90 & 4.17 & 3.13 & $10^{-}$ \\
\hline 7.943 & 59.88 & 49.7 & 19.2 & 14.47 & 59.82 & 49.6 & 19.27 & 14.45 & 59.86 & 49.62 & 19.25 & 14.46 & $10^{-2}$ \\
\hline 10.00 & 30.52 & 25.01 & 8.88 & 6.68 & 30.51 & 25.00 & 8.88 & 6.68 & 30.52 & 25.00 & 8.87 & 6.68 & $10^{-2}$ \\
\hline
\end{tabular}


TABLE VIII: Neutrino flux $\left(\mathrm{m}^{-2} \mathrm{sec}^{-1} \mathrm{sr}^{-1} \mathrm{GeV}^{-1}\right)$ for $0.4 \geq \cos \theta_{z}>0.3$

\begin{tabular}{|c|c|c|c|c|c|c|c|c|c|c|c|c|c|}
\hline & & Kam & nioka & & & Sudb & ury & & & Gran & Sasso & & \\
\hline$E_{\nu}(\mathrm{GeV})$ & $\nu_{\mu}$ & $\bar{\nu}_{\mu}$ & $\nu_{e}$ & $\bar{\nu}_{e}$ & $\nu_{\mu}$ & $\bar{\nu}_{\mu}$ & $\nu_{e}$ & $\bar{\nu}_{e}$ & $\nu_{\mu}$ & $\bar{\nu}_{\mu}$ & $\nu_{e}$ & $\bar{\nu}_{e}$ & Norm \\
\hline .1000 & 5.10 & 5.16 & 2.54 & 2.37 & 14.14 & 14.03 & 7.56 & 5.68 & 8.88 & 8.91 & 4.46 & $\begin{array}{ll}6 & 3.97\end{array}$ & $10^{3}$ \\
\hline .1259 & 39.93 & 40.41 & 20.02 & 18.52 & 107.11 & 106.64 & 57.68 & 43.54 & 68.83 & 68.89 & 34.82 & 230.77 & $10^{2}$ \\
\hline .1585 & 30.50 & 30.69 & 15.28 & 13.97 & 78.28 & 78.02 & 42.24 & 31.96 & 51.63 & 51.71 & 26.12 & 222.99 & $10^{2}$ \\
\hline .1995 & 22.50 & 22.61 & 11.37 & 10.27 & 54.48 & 54.68 & 29.70 & 22.65 & 37.39 & 37.35 & 19.00 & 016.63 & $10^{2}$ \\
\hline .2512 & 15.98 & 16.11 & 8.22 & 7.37 & 36.00 & 36.47 & 20.05 & 15.43 & 25.98 & 25.91 & 13.45 & 511.64 & $10^{2}$ \\
\hline .3162 & 11.05 & 11.06 & 5.74 & 5.10 & 22.92 & 23.33 & 13.04 & 10.12 & 17.35 & 17.34 & $\begin{array}{l}19.19 \\
\end{array}$ & 7.90 & $10^{2}$ \\
\hline .3981 & 7.48 & 7.45 & 3.92 & 3.43 & 14.25 & 14.46 & 8.17 & 6.43 & 11.33 & 11.28 & 6.08 & 3.18 & $10^{2}$ \\
\hline .5012 & 4.97 & 4.95 & 2.62 & 2.26 & 8.69 & 8.76 & 4.97 & 3.97 & 7.26 & 7.15 & 3.91 & 3.30 & $10^{2}$ \\
\hline .6310 & 32.46 & 31.82 & 17.09 & 14.63 & 51.90 & 51.99 & 29.69 & 23.67 & 45.18 & 44.34 & 24.49 & 20.58 & $10^{1}$ \\
\hline .7943 & 20.65 & 20.06 & 10.88 & 9.22 & 30.39 & 30.23 & 17.39 & 13.83 & 27.48 & 26.89 & 14.98 & 312.40 & $10^{1}$ \\
\hline 1.000 & 12.81 & 12.43 & 6.78 & 5.65 & 17.48 & 17.24 & 9.97 & 7.91 & 16.35 & 15.93 & 8.94 & $4 \quad 7.25$ & $10^{1}$ \\
\hline 1.259 & 7.78 & 7.50 & 4.11 & 3.37 & 9.88 & 9.68 & 5.58 & 4.37 & 9.46 & 9.19 & 5.20 & 4.16 & $10^{1}$ \\
\hline 1.585 & 46.19 & 43.94 & 24.12 & 19.49 & 55.21 & 53.30 & 30.51 & 23.59 & 53.74 & 51.55 & 29.21 & 23.10 & 1 \\
\hline 1.995 & 26.76 & 25.06 & 13.68 & 10.91 & 30.50 & 28.81 & 16.30 & 12.49 & 29.98 & 28.16 & 15.80 & 12.32 & 1 \\
\hline 2.512 & 15.21 & 13.98 & 7.50 & 5.89 & 16.57 & 15.42 & 8.55 & 6.49 & 16.27 & 15.03 & 8.25 & $5 \quad 6.36$ & 1 \\
\hline 3.162 & 8.38 & 7.59 & 3.97 & 3.09 & 8.89 & 8.11 & 4.38 & 3.28 & 8.74 & 7.94 & 14.24 & $\begin{array}{l}4.24 \\
\end{array}$ & 1 \\
\hline 3.981 & 44.88 & 40.07 & 20.30 & 15.66 & 47.11 & 41.80 & 21.82 & 16.19 & 46.49 & 41.46 & 21.39 & 16.25 & $10^{-1}$ \\
\hline 5.012 & 23.67 & 20.76 & 10.10 & 7.70 & 24.47 & 21.29 & 10.54 & 7.87 & 24.14 & 21.22 & 10.49 & 7.90 & $10^{-1}$ \\
\hline 6.310 & 12.32 & 10.64 & 4.91 & 3.70 & 12.47 & 10.74 & 4.98 & 3.74 & 12.38 & 10.72 & 4.98 & 3.74 & $10^{-1}$ \\
\hline 7.943 & 63.40 & 54.11 & 23.35 & 17.54 & 63.19 & 53.98 & 23.26 & 17.49 & 63.33 & 54.03 & 23.25 & 17.49 & $10^{-2}$ \\
\hline 10.00 & 32.32 & 27.25 & 10.90 & 8.19 & 32.29 & 27.23 & 10.88 & 8.18 & 32.31 & 27.23 & 10.88 & 8.18 & $10^{-2}$ \\
\hline
\end{tabular}


TABLE IX: Neutrino flux $\left(\mathrm{m}^{-2} \mathrm{sec}^{-1} \mathrm{sr}^{-1} \mathrm{GeV}^{-1}\right)$ for $0.3 \geq \cos \theta_{z}>0.2$

\begin{tabular}{|c|c|c|c|c|c|c|c|c|c|c|c|c|c|}
\hline & & $\mathrm{Ka}$ & nioka & & & Sudl & bury & & & Gran & I Sasso & & \\
\hline$E_{\nu}(\mathrm{GeV})$ & $\nu_{\mu}$ & $\bar{\nu}_{\mu}$ & $\nu_{e}$ & $\bar{\nu}_{e}$ & $\nu_{\mu}$ & $\bar{\nu}_{\mu}$ & $\nu_{e}$ & $\bar{\nu}_{e}$ & $\nu_{\mu}$ & $\bar{\nu}_{\mu}$ & $\nu_{e}$ & $\bar{\nu}_{e}$ & Norm \\
\hline .1000 & 6.23 & 6.30 & 3.08 & 2.86 & 17.48 & 17.38 & 9.28 & 6.98 & 10.91 & 10.93 & 3.41 & 4.85 & $10^{3}$ \\
\hline .1259 & 4.76 & 4.82 & 2.38 & 2.19 & 12.95 & 12.93 & 6.96 & 5.23 & 8.25 & 8.25 & 5.13 & 3.66 & $10^{3}$ \\
\hline .1585 & 35.35 & 35.73 & 17.81 & 16.13 & 91.76 & 92.00 & 49.75 & 37.59 & 60.22 & 60.32 & 30.42 & 26.74 & $10^{2}$ \\
\hline .1995 & 25.28 & 25.54 & 12.92 & 11.56 & 61.70 & 62.21 & 34.07 & 25.97 & 42.25 & 42.33 & 21.64 & $=18.87$ & $10^{2}$ \\
\hline .2512 & 17.43 & 17.63 & 9.11 & 8.07 & 39.57 & 40.15 & 22.44 & 17.30 & 28.40 & 28.48 & 14.90 & 12.88 & $10^{2}$ \\
\hline .3162 & 11.75 & 11.85 & 6.28 & 5.48 & 24.59 & 25.07 & 14.19 & 11.10 & 18.51 & 18.56 & 9.97 & 8.57 & $10^{2}$ \\
\hline .3981 & 7.81 & 7.84 & 4.23 & 3.63 & 14.99 & 15.24 & 8.71 & 6.92 & 11.83 & 11.81 & 6.46 & 5.51 & $10^{2}$ \\
\hline .5012 & 5.12 & 5.11 & 2.78 & 2.36 & 9.02 & 9.09 & 5.25 & 4.21 & 7.45 & 7.39 & 4.08 & 3.45 & $10^{2}$ \\
\hline .6310 & 32.99 & 32.67 & 17.94 & 15.15 & 53.50 & 53.53 & 31.10 & 24.97 & 46.08 & 45.44 & 425.43 & 21.30 & $10^{1}$ \\
\hline .7943 & 20.84 & 20.51 & 11.44 & 9.55 & 31.26 & 31.02 & 18.09 & 14.54 & 27.86 & 27.37 & 715.54 & $=12.88$ & $10^{1}$ \\
\hline 1.000 & 12.93 & 12.66 & 7.15 & 5.88 & 17.97 & 17.70 & 10.39 & 8.33 & 16.48 & 16.14 & 9.28 & 7.58 & $10^{1}$ \\
\hline 1.259 & 7.90 & 7.66 & 4.32 & 3.53 & 10.17 & 10.00 & 5.94 & 4.68 & 9.59 & 9.35 & 5.43 & 4.37 & $10^{1}$ \\
\hline 1.585 & 4.72 & 4.53 & 2.55 & 2.06 & 5.73 & 5.59 & 3.32 & 2.59 & 5.51 & 5.31 & 3.10 & 2.44 & $10^{1}$ \\
\hline 1.995 & 27.47 & 26.18 & 14.8 & 11.76 & 31.97 & 30.6 & 17.96 & 14.02 & 31.12 & 29. & 17.17 & 13.31 & 1 \\
\hline 2.512 & 15.73 & 14.77 & 8.36 & 6.53 & 17.39 & 16.42 & 9.55 & 7.32 & 17.06 & 15.96 & 9.16 & 7.11 & 1 \\
\hline 3.162 & 8.77 & 8.10 & 4.51 & 3.51 & 9.37 & 8.73 & 4.99 & 3.76 & 9.21 & 8.51 & 4.82 & 3.71 & 1 \\
\hline 3.981 & 47.46 & 43.32 & 23.42 & 18.24 & 50.09 & 45.94 & 25.44 & 19.03 & 49.16 & 44.75 & 24.91 & 18.84 & $10^{-1}$ \\
\hline 5.012 & 25.19 & 22.79 & 11.96 & 9.15 & 26.03 & 23.48 & 12.54 & 9.37 & 25.80 & 23.21 & 12.36 & 9.37 & $10^{-1}$ \\
\hline 6.310 & 13.17 & 11.78 & 5.97 & 4.49 & 13.30 & 11.85 & 6.04 & 4.53 & 13.29 & 11.84 & 6.00 & 4.54 & $10^{-1}$ \\
\hline 7.943 & 6.80 & 6.00 & 2.90 & 2.18 & 6.78 & 5.99 & 2.89 & 2.17 & 6.78 & 5.99 & 2.89 & 2.17 & $10^{-1}$ \\
\hline 10.00 & 34.82 & 30.38 & 13.83 & 10.38 & 34.80 & 30.37 & 13.82 & 10.37 & 34.80 & 30.37 & 13.82 & 10.37 & $10^{-2}$ \\
\hline
\end{tabular}


TABLE X: Neutrino flux $\left(\mathrm{m}^{-2} \mathrm{sec}^{-1} \mathrm{sr}^{-1} \mathrm{GeV}^{-1}\right)$ for $0.2 \geq \cos \theta_{z}>0.1$

\begin{tabular}{|c|c|c|c|c|c|c|c|c|c|c|c|c|c|}
\hline & & Kam & nioka & & & Sudl & bury & & & Gran & I Sasso & & \\
\hline$E_{\nu}(\mathrm{GeV})$ & $\nu_{\mu}$ & $\bar{\nu}_{\mu}$ & $\nu_{e}$ & $\bar{\nu}_{e}$ & $\nu_{\mu}$ & $\bar{\nu}_{\mu}$ & $\nu_{e}$ & $\bar{\nu}_{e}$ & $\nu_{\mu}$ & $\bar{\nu}_{\mu}$ & $\nu_{e}$ & $\bar{\nu}_{e}$ & Norm \\
\hline .1000 & 8.66 & 8.76 & 4.23 & 3.92 & 24.56 & 24.41 & 12.94 & 9.70 & 15.19 & 15.25 & 7.46 & 6.67 & $10^{3}$ \\
\hline .1259 & 6.46 & 6.52 & 3.19 & 2.93 & 17.73 & 17.69 & 9.47 & 7.12 & 11.24 & 11.23 & 5.58 & 4.93 & $10^{3}$ \\
\hline .1585 & 4.62 & 4.68 & 2.33 & 2.09 & 12.11 & 12.17 & 6.59 & 4.97 & 7.92 & 7.93 & 3.99 & 3.51 & $10^{3}$ \\
\hline .1995 & 31.75 & 32.26 & 16.38 & 14.56 & 78.09 & 79.26 & 43.65 & 33.38 & 53.16 & 53.57 & 27.59 & 24.01 & $10^{2}$ \\
\hline .2512 & 21.09 & 21.42 & 11.19 & 9.86 & 47.95 & 49.10 & 27.65 & 21.50 & 34.40 & 34.61 & 18.43 & 15.82 & $10^{2}$ \\
\hline .3162 & 13.69 & 13.92 & 7.43 & 6.44 & 28.67 & 29.46 & 16.84 & 13.30 & 21.58 & 21.70 & 11.80 & 10.11 & $10^{2}$ \\
\hline .3981 & 8.80 & 8.92 & 4.87 & 4.14 & 16.94 & 17.29 & 10.00 & 8.03 & 13.33 & 13.36 & 7.40 & 6.31 & $10^{2}$ \\
\hline .5012 & 5.63 & 5.64 & 3.15 & 2.64 & 9.95 & 10.02 & 5.85 & 4.76 & 8.15 & 8.11 & 4.58 & 3.87 & $10^{2}$ \\
\hline .6310 & 35.63 & 35.33 & 19.86 & 16.45 & 57.78 & 57.69 & 33.95 & 27.47 & 49.10 & 48.61 & 27.90 & 23.40 & $10^{1}$ \\
\hline .7943 & 22.06 & 21.89 & 12.39 & 10.11 & 33.31 & 32.92 & 19.53 & 15.76 & 29.18 & 28.74 & 16.68 & 13.82 & $10^{1}$ \\
\hline 1.000 & 13.38 & 13.40 & 7.68 & 6.15 & 19.00 & 18.61 & 11.17 & 8.99 & 17.10 & 16.80 & 9.84 & 8.00 & $10^{1}$ \\
\hline 1.259 & 8.08 & 8.04 & 4.67 & 3.69 & 10.67 & 10.46 & 6.37 & 5.01 & 9.85 & 9.69 & 5.77 & 4.61 & $10^{1}$ \\
\hline 1.585 & 4.83 & 4.72 & 2.77 & 2.18 & 5.96 & 5.82 & 3.56 & 2.76 & 5.63 & 5.47 & 3.28 & 2.62 & $10^{1}$ \\
\hline 1.995 & 28.31 & 27.16 & 15.9 & 12.63 & 33.19 & 32.00 & 19.45 & 15.11 & 31.86 & 30.39 & 18.18 & 14.51 & 1 \\
\hline 2.512 & 16.21 & 15.43 & 9.06 & 7.10 & 18.30 & 17.40 & 10.53 & 8.10 & 17.70 & 16.74 & 9.99 & 7.75 & 1 \\
\hline 3.162 & 9.10 & 8.57 & 5.02 & 3.89 & 9.95 & 9.40 & 5.64 & 4.27 & 9.68 & 9.08 & 5.41 & 4.11 & 1 \\
\hline 3.981 & 5.00 & 4.64 & 2.70 & 2.07 & 5.32 & 5.00 & 2.96 & 2.21 & 5.22 & 4.83 & 2.87 & 2.17 & 1 \\
\hline 5.012 & 26.82 & 24.67 & 14.19 & 10.71 & 27.86 & 25.86 & 15.03 & 11.15 & 27.67 & 25.38 & 14.82 & 11.08 & $10^{-1}$ \\
\hline 6.310 & 14.18 & 13.02 & 7.29 & 5.48 & 14.38 & 13.22 & 7.44 & 5.56 & 14.38 & 13.17 & 7.42 & 5.56 & $10^{-1}$ \\
\hline 7.943 & 7.42 & 6.80 & 3.67 & 2.77 & 7.39 & 6.78 & 3.65 & 2.75 & 7.39 & 6.78 & 3.65 & 2.76 & $10^{-1}$ \\
\hline 10.00 & 38.24 & 34.80 & 17.98 & 13.52 & 38.20 & 34.76 & 17.96 & 13.50 & 38.20 & 34.77 & 17.96 & 13.50 & $10^{-2}$ \\
\hline
\end{tabular}


TABLE XI: Neutrino flux $\left(\mathrm{m}^{-2} \mathrm{sec}^{-1} \mathrm{sr}^{-1} \mathrm{GeV}^{-1}\right)$ for $0.1 \geq \cos \theta_{z}>0.0$

\begin{tabular}{|c|c|c|c|c|c|c|c|c|c|c|c|c|c|}
\hline & & Kar & nioka & & & Sudl & bury & & & Gran & Sasso & & \\
\hline$E_{\nu}(\mathrm{GeV})$ & $\nu_{\mu}$ & $\bar{\nu}_{\mu}$ & $\nu_{e}$ & $\bar{\nu}_{e}$ & $\nu_{\mu}$ & $\bar{\nu}_{\mu}$ & $\nu_{e}$ & $\bar{\nu}_{e}$ & $\nu_{\mu}$ & $\bar{\nu}_{\mu}$ & $\nu_{e}$ & $\bar{\nu}_{e}$ & Norm \\
\hline .1000 & 4.19 & 14.40 & 6.85 & 6.38 & 39.91 & 39.56 & 20.65 & 15.71 & 24.89 & 24.87 & 12.08 & 10.70 & $10^{3}$ \\
\hline .1259 & 10.27 & 10.43 & 5.01 & 4.62 & 28.11 & 27.96 & 14.85 & 11.23 & 17.85 & 17.85 & 8.78 & 7.77 & $10^{3}$ \\
\hline .1585 & 7.10 & 7.19 & 3.54 & 3.21 & 18.41 & 18.60 & 10.01 & 7.59 & 12.09 & 12.13 & 6.12 & 5.35 & $10^{3}$ \\
\hline .1995 & 4.68 & 4.73 & 2.40 & 2.14 & 11.30 & 11.60 & 6.41 & 4.90 & 7.75 & 7.80 & 4.07 & 3.52 & $10^{3}$ \\
\hline .2512 & 29.50 & 30.04 & 15.88 & 13.70 & 66.33 & 68.44 & 39.32 & 30.33 & 47.63 & 47.94 & 25.83 & 22.23 & $10^{2}$ \\
\hline .3162 & 18.20 & 18.53 & 10.09 & 8.67 & 37.91 & 39.19 & 22.90 & 18.05 & 28.51 & 28.77 & 16.00 & 13.60 & $10^{2}$ \\
\hline .3981 & 11.19 & 11.35 & 6.33 & 5.37 & 21.48 & 22.03 & 12.96 & 10.44 & 16.87 & 16.97 & 9.65 & 8.13 & $10^{2}$ \\
\hline .5012 & 6.87 & 6.94 & 3.94 & 3.24 & 12.13 & 12.28 & 7.25 & 5.91 & 9.93 & 9.89 & 5.72 & 4.77 & $10^{2}$ \\
\hline .6310 & 41.55 & 42.02 & 23.91 & 19.37 & 67.96 & 68.49 & 40.39 & 32.94 & 58.02 & 57.33 & 33.87 & 27.43 & $10^{1}$ \\
\hline .7943 & 24.80 & 25.10 & 14.52 & 11.59 & 37.98 & 37.79 & 22.71 & 18.41 & 33.41 & 32.91 & 19.66 & 15.80 & $10^{1}$ \\
\hline 1.000 & 14.74 & 14.82 & 8.82 & 6.89 & 21.07 & 20.77 & 12.81 & 10.25 & 18.97 & 18.66 & 11.18 & 9.11 & $10^{1}$ \\
\hline 1.259 & 8.77 & 8.70 & 5.24 & 4.00 & 11.53 & 11.56 & 7.08 & 5.56 & 10.70 & 10.49 & 6.37 & 5.15 & $10^{1}$ \\
\hline 1.585 & 5.09 & 5.07 & 3.05 & 2.33 & 6.39 & 6.34 & 3.86 & 3.00 & 5.96 & 5.87 & 3.62 & 2.86 & $10^{1}$ \\
\hline 1.995 & $29.0^{\prime}$ & 29.19 & 17.5 & 13.62 & 35.52 & 34.0 & 21.02 & 16.35 & 33.00 & 32 & 20.26 & 15.64 & 1 \\
\hline 2.512 & 16.79 & 16.54 & 10.11 & 7.69 & 19.13 & 18.35 & 11.43 & 8.86 & 18.31 & 17.82 & 10.98 & 8.46 & 1 \\
\hline 3.162 & 9 & 9.17 & 5.58 & 4.28 & 10.34 & 9.87 & 6.18 & 4.72 & 10.14 & $\begin{array}{r}9.59 \\
-\quad 5\end{array}$ & 5.89 & 4.55 & 1 \\
\hline 3.981 & 5.18 & 4.97 & 2.98 & 2.34 & 5.61 & 5.27 & 3.30 & 2.49 & 5.53 & 5.11 & 3.14 & 2.41 & 1 \\
\hline 5.012 & 28.10 & 26.53 & 16.1 & 12.29 & 29.44 & 27.89 & 17.16 & 13.06 & 29.3 & 27.37 & 16.43 & 12.52 & $10^{-1}$ \\
\hline 6.310 & 15.09 & 14.18 & 8.57 & 6.39 & 15.26 & 14.51 & 8.71 & 6.62 & 15.28 & 14.43 & 8.53 & 6.45 & $10^{-1}$ \\
\hline 7.943 & 7.98 & 7.54 & 4.40 & 3.32 & 7.96 & 7.49 & 4.38 & 3.29 & 7.96 & 7.50 & 4.41 & 3.31 & $10^{-1}$ \\
\hline 10.00 & 41.72 & 39.25 & 22.42 & 16.87 & 41.70 & 39.17 & 22.40 & 16.82 & 41.69 & 39.19 & 22.43 & 16.86 & $10^{-2}$ \\
\hline
\end{tabular}


TABLE XII: Neutrino flux $\left(\mathrm{m}^{-2} \mathrm{sec}^{-1} \mathrm{sr}^{-1} \mathrm{GeV}^{-1}\right)$ for $0.0 \geq \cos \theta_{z}>-0.1$

\begin{tabular}{|c|c|c|c|c|c|c|c|c|c|c|c|c|c|}
\hline & & Kar & nioka & & & Sudl & lbury & & & Gran & Sasso & & \\
\hline$E_{\nu}(\mathrm{GeV})$ & $\nu_{\mu}$ & $\bar{\nu}_{\mu}$ & $\nu_{e}$ & $\bar{\nu}_{e}$ & $\nu_{\mu}$ & $\bar{\nu}_{\mu}$ & $\nu_{e}$ & $\bar{\nu}_{e}$ & $\nu_{\mu}$ & $\bar{\nu}_{\mu}$ & $\nu_{e}$ & $\bar{\nu}_{e}$ & Norm \\
\hline .1000 & 5.16 & 15.36 & 7.33 & 6.74 & 38.57 & 38.36 & 19.77 & 15.33 & 24.81 & 24.96 & 12.23 & 10.64 & $10^{3}$ \\
\hline .1259 & 11.02 & 11.06 & 5.33 & 4.89 & 27.25 & 27.11 & 14.22 & 11.01 & 17.84 & 17.89 & 8.92 & 7.72 & $10^{3}$ \\
\hline .1585 & 7.55 & 7.59 & 3.76 & 3.39 & 17.94 & 18.07 & 9.68 & 7.50 & 12.09 & 12.16 & 6.16 & 5.28 & $10^{3}$ \\
\hline .1995 & 4.91 & 4.96 & 2.54 & 2.26 & 11.12 & 11.34 & 6.24 & 4.85 & 7.70 & 7.78 & 4.07 & 3.45 & $10^{3}$ \\
\hline .2512 & 30.78 & 31.12 & 16.47 & 14.43 & 65.79 & 67.71 & 38.40 & 30.08 & 46.67 & 47.52 & 25.89 & 21.83 & $10^{2}$ \\
\hline .3162 & 18.71 & 19.04 & 10.41 & 8.96 & 37.76 & 39.04 & 22.57 & 18.03 & 27.81 & 28.35 & 15.88 & 13.31 & $10^{2}$ \\
\hline .3981 & 11.34 & 11.57 & 6.46 & 5.47 & 21.55 & 22.14 & 12.89 & 10.49 & 16.42 & 16.67 & 9.48 & 7.93 & $10^{2}$ \\
\hline .5012 & 6.91 & 7.01 & 3.96 & 3.29 & 12.28 & 12.44 & 7.29 & 5.96 & 9.64 & 9.71 & 5.60 & 4.66 & $10^{2}$ \\
\hline .6310 & 41.61 & 41.96 & 24.00 & 19.50 & 68.99 & 69.10 & 41.29 & 33.33 & 56.39 & 56.08 & 33.05 & 27.08 & $10^{1}$ \\
\hline .7943 & 24.87 & 24.93 & 14.61 & 11.51 & 38.58 & 38.27 & 23.24 & 18.60 & 32.38 & 32.21 & 19.29 & 15.64 & $10^{1}$ \\
\hline 1.000 & 14.73 & 14.74 & 8.83 & 6.80 & 21.54 & 21.21 & 12.93 & 10.33 & 18.42 & 18.39 & 11.09 & 8.93 & $10^{1}$ \\
\hline 1.259 & 8.58 & 8.63 & 5.17 & 4.02 & 11.89 & 11.69 & 7.15 & 5.65 & 10.62 & 10.41 & 6.32 & 5.00 & $10^{1}$ \\
\hline 1.585 & 5.00 & 4.94 & 3.00 & 2.33 & 6.49 & 6.30 & 3.92 & 3.06 & 6.01 & 5.82 & 3.55 & 2.80 & $10^{1}$ \\
\hline 1.995 & 29.08 & 28.13 & 17.4 & 13.35 & 35.29 & 33.72 & 21.34 & 16.50 & 33.15 & 32.25 & 19.79 & 15.56 & 1 \\
\hline 2.512 & 16.68 & 16.31 & 9.99 & 7.59 & 19.21 & 18.46 & 11.55 & 8.86 & 18.37 & 17.77 & 10.99 & 8.40 & 1 \\
\hline 3.162 & 9 & 9.18 & 5.52 & 4.23 & 10.43 & 9.92 & 6.21 & 4.71 & 10.12 & 9.63 & 5.97 & 4.50 & 1 \\
\hline 3.981 & 5.19 & 4.95 & 2.97 & 2.31 & 5.57 & 5.21 & 3.29 & 2.47 & 5.50 & 5.14 & 3.16 & 2.41 & 1 \\
\hline 5.012 & 28.28 & 26.40 & 15.95 & 12.39 & 29.05 & 27.81 & 17.03 & 12.87 & 29.33 & 27.41 & 16.51 & 12.58 & $10^{-1}$ \\
\hline 6.310 & 15.14 & 14.15 & 8.49 & 6.47 & 15.15 & 14.56 & 8.65 & 6.55 & 15.33 & 14.42 & 8.56 & 6.47 & $10^{-1}$ \\
\hline 7.943 & 7.98 & 7.54 & 4.41 & 3.30 & 7.98 & 7.48 & 4.39 & 3.30 & 7.95 & 7.50 & 4.41 & 3.31 & $10^{-1}$ \\
\hline 10.00 & 41.71 & 39.25 & 22.44 & 16.85 & 41.72 & 39.16 & 22.41 & 16.84 & 41.67 & 39.19 & 22.43 & 16.85 & $10^{-2}$ \\
\hline
\end{tabular}


TABLE XIII: Neutrino flux $\left(\mathrm{m}^{-2} \mathrm{sec}^{-1} \mathrm{sr}^{-1} \mathrm{GeV}^{-1}\right)$ for $-0.1 \geq \cos \theta_{z}>-0.2$

\begin{tabular}{|c|c|c|c|c|c|c|c|c|c|c|c|c|c|}
\hline & & $\mathrm{Ka}$ & iioka & & & Sudl & bury & & & Gran & I Sasso & & \\
\hline$E_{\nu}(\mathrm{GeV})$ & $\nu_{\mu}$ & $\bar{\nu}_{\mu}$ & $\nu_{e}$ & $\bar{\nu}_{e}$ & $\nu_{\mu}$ & $\bar{\nu}_{\mu}$ & $\nu_{e}$ & $\bar{\nu}_{e}$ & $\nu_{\mu}$ & $\bar{\nu}_{\mu}$ & $\nu_{e}$ & $\bar{\nu}_{e}$ & Norm \\
\hline .1000 & 10.87 & 10.94 & 5.36 & 4.76 & 21.25 & 21.17 & 10.95 & 8.70 & 15.11 & 15.14 & 47.68 & 6.37 & $10^{3}$ \\
\hline .1259 & 8.00 & 8.03 & 3.99 & 3.51 & 15.49 & 15.48 & 8.09 & 6.42 & 11.05 & 11.11 & 5.70 & 4.69 & $10^{3}$ \\
\hline .1585 & 5.61 & 5.66 & 2.85 & 2.50 & 10.71 & 10.75 & 5.70 & 4.53 & 7.70 & 7.76 & 4.04 & 3.31 & $10^{3}$ \\
\hline .1995 & 37.64 & 38.05 & 19.62 & 17.06 & 70.25 & 71.21 & 38.50 & 30.55 & 51.06 & 51.75 & 27.49 & 22.55 & $10^{2}$ \\
\hline .2512 & 24.34 & 24.56 & 13.07 & 11.24 & 44.20 & 45.09 & 24.94 & 19.91 & 32.48 & 33.15 & 18.01 & 14.83 & $10^{2}$ \\
\hline .3162 & 15.28 & 15.46 & 8.41 & 7.17 & 27.06 & 27.62 & 15.58 & 12.54 & 20.15 & 20.61 & 11.43 & 9.43 & $10^{2}$ \\
\hline .3981 & 9.52 & 9.61 & 5.32 & 4.51 & 16.27 & 16.56 & 9.47 & 7.66 & 12.37 & 12.58 & 7.08 & 5.84 & $10^{2}$ \\
\hline .5012 & 5.93 & 5.94 & 3.33 & 2.80 & 9.68 & 9.79 & 5.65 & 4.57 & 7.56 & 7.60 & 4.31 & 3.56 & $10^{2}$ \\
\hline .6310 & 36.40 & 36.35 & 20.38 & 17.12 & 57.34 & 57.21 & 33.36 & 27.03 & 45.69 & 45.65 & 26.18 & 21.50 & $10^{1}$ \\
\hline .7943 & 22.17 & 22.02 & 12.58 & 10.37 & 33.32 & 32.95 & 19.58 & 15.79 & 27.16 & 27.08 & 15.73 & 12.87 & $10^{1}$ \\
\hline 1.000 & 13.40 & 13.24 & 7.74 & 6.23 & 18.96 & 18.73 & 11.37 & 9.06 & 15.94 & 15.86 & 9.32 & 7.58 & $10^{1}$ \\
\hline 1.259 & 7.97 & 7.92 & 4.58 & 3.68 & 10.69 & 10.55 & 6.45 & 5.08 & 9.35 & 9.23 & 5.46 & 4.36 & $10^{1}$ \\
\hline 1.585 & 4.69 & 4.62 & 2.69 & 2.14 & 5.99 & 5.90 & 3.59 & 2.80 & 5.45 & 5.28 & 3.16 & 2.48 & $10^{1}$ \\
\hline 1.995 & 27.37 & 26.40 & 15.7 & 12.30 & 33.33 & 32.45 & 19.6 & 15.25 & 31.10 & 29.68 & 17.89 & 13.99 & 1 \\
\hline 2.512 & 15.85 & 15.14 & 8.86 & 6.97 & 18.35 & 17.53 & 10.61 & 8.14 & 17.32 & 16.52 & 9.81 & 7.70 & 1 \\
\hline 3.162 & 8 & 8.46 & 4.90 & 3.84 & 7 & 9.41 & 5.66 & 4.29 & 9.56 & 9.02 & 5.31 & 4.11 & 1 \\
\hline 3.981 & 4.92 & 4.59 & 2.68 & 2.05 & 5.33 & 4.99 & 2.95 & 2.23 & 5.20 & 4.81 & 2.84 & 2.14 & 1 \\
\hline 5.012 & 26.76 & 24.77 & 14.11 & 10.78 & 27.93 & 25.76 & 14.91 & 11.27 & 27.41 & 25.34 & 14.67 & 10.95 & $10^{-1}$ \\
\hline 6.310 & 14.24 & 13.11 & 7.27 & 5.52 & 14.40 & 13.19 & 7.40 & 5.59 & 14.26 & 13.17 & 7.37 & 5.53 & $10^{-1}$ \\
\hline 7.943 & 7.41 & 6.79 & 3.67 & 2.76 & 7.39 & 6.78 & 3.65 & 2.75 & 7.41 & 6.78 & 3.66 & 2.76 & $10^{-1}$ \\
\hline 10.00 & 38.22 & 34.77 & 17.99 & 13.51 & 38.20 & 34.77 & 17.96 & 13.50 & 38.23 & 34.77 & 17.97 & 13.51 & $10^{-2}$ \\
\hline
\end{tabular}


TABLE XIV: Neutrino flux $\left(\mathrm{m}^{-2} \mathrm{sec}^{-1} \mathrm{sr}^{-1} \mathrm{GeV}^{-1}\right)$ for $-0.2 \geq \cos \theta_{z}>-0.3$

\begin{tabular}{|c|c|c|c|c|c|c|c|c|c|c|c|c|c|}
\hline & & $\mathrm{Ka}$ & nioka & & & Sudl & bury & & & Gran & I Sasso & & \\
\hline$E_{\nu}(\mathrm{GeV})$ & $\nu_{\mu}$ & $\bar{\nu}_{\mu}$ & $\nu_{e}$ & $\bar{\nu}_{e}$ & $\nu_{\mu}$ & $\bar{\nu}_{\mu}$ & $\nu_{e}$ & $\bar{\nu}_{e}$ & $\nu_{\mu}$ & $\bar{\nu}_{\mu}$ & $\nu_{e}$ & $\bar{\nu}_{e}$ & Norm \\
\hline .1000 & 8.65 & 8.65 & 4.41 & 3.71 & 12.70 & 12.71 & 6.59 & 5.30 & 10.35 & 10.37 & $7 \quad 5.34$ & 4.36 & $10^{3}$ \\
\hline .1259 & 6.49 & 6.50 & 3.35 & 2.80 & 9.52 & 9.54 & 4.99 & 4.01 & 7.77 & 7.80 & 4.06 & 3.29 & $10^{3}$ \\
\hline .1585 & 4.68 & 4.70 & 2.44 & 2.04 & 6.87 & 6.91 & 3.64 & 2.91 & 5.60 & 5.64 & $4 \quad 2.96$ & 2.39 & $10^{3}$ \\
\hline .1995 & 32.35 & 32.56 & 17.09 & 14.30 & 47.44 & 47.87 & 25.52 & 20.46 & 38.70 & 39.10 & 20.76 & 16.82 & $10^{2}$ \\
\hline .2512 & 21.46 & 21.64 & 11.59 & 9.71 & 31.32 & 31.76 & 17.19 & 13.94 & 25.67 & 26.09 & 14.07 & 11.44 & $10^{2}$ \\
\hline .3162 & 13.89 & 14.02 & 7.63 & 6.41 & 20.09 & 20.38 & 11.26 & 9.16 & 16.53 & 16.84 & 49.20 & 7.56 & $10^{2}$ \\
\hline .3981 & 8.86 & 8.93 & 4.91 & 4.12 & 12.65 & 12.81 & 7.17 & 5.84 & 10.46 & 10.60 & 5.89 & 4.84 & $10^{2}$ \\
\hline .5012 & 5.59 & 5.60 & 3.10 & 2.59 & 7.86 & 7.92 & 4.47 & 3.64 & 6.54 & 6.57 & 3.72 & 3.02 & $10^{2}$ \\
\hline .6310 & 34.86 & 34.64 & 19.40 & 16.11 & 48.10 & 47.87 & 27.56 & 22.19 & 40.47 & 40.47 & 22.95 & 18.66 & $10^{1}$ \\
\hline .7943 & 21.47 & 21.20 & 11.94 & 9.91 & 28.88 & 28.52 & 16.59 & 13.27 & 24.56 & 24.44 & 13.96 & 11.31 & $10^{1}$ \\
\hline 1.000 & 13.06 & 12.82 & 7.25 & 5.97 & 16.99 & 16.75 & 9.75 & 7.79 & 14.66 & 14.50 & 8.37 & 6.71 & $10^{1}$ \\
\hline 1.259 & 7.84 & 7.62 & 4.37 & 3.51 & 9.80 & 9.62 & 5.65 & 4.50 & 8.68 & 8.55 & 4.91 & 3.91 & $10^{1}$ \\
\hline 1.585 & 4.61 & 4.46 & 2.56 & 2.03 & 5.57 & 5.43 & 3.20 & 2.52 & 5.06 & 4.92 & 2.83 & 2.24 & $10^{1}$ \\
\hline 1.995 & 26.72 & 25.62 & 14.4 & 11.52 & 31.28 & 30.02 & 17.61 & 13.67 & 28.98 & 27.67 & 15.97 & 12.54 & 1 \\
\hline 2.512 & 15.33 & 14.39 & 8.07 & 6.37 & 17.34 & 16.25 & 9.39 & 7.25 & 16.25 & 15.34 & 8.77 & 6.80 & 1 \\
\hline 3.162 & 8.57 & 7.89 & 4.39 & 3.42 & 9.40 & 8.69 & 4.93 & 3.76 & 8.95 & 8.34 & 4.67 & 3.59 & 1 \\
\hline 3.981 & 46.56 & 42.34 & 23.13 & 17.85 & 49.82 & 45.82 & 25.35 & 19.02 & 48.32 & 44.27 & 24.19 & 18.46 & $10^{-1}$ \\
\hline 5.012 & 24.98 & 22.40 & 11.84 & 9.06 & 25.96 & 23.42 & 12.52 & 9.36 & 25.53 & 23.05 & 12.22 & 9.25 & $10^{-1}$ \\
\hline 6.310 & 13.16 & 11.70 & 5.93 & 4.49 & 13.31 & 11.83 & 6.04 & 4.53 & 13.24 & 11.81 & 6.01 & 4.52 & $10^{-1}$ \\
\hline 7.943 & 6.80 & 6.01 & 2.90 & 2.18 & 6.78 & 5.99 & 2.89 & 2.17 & 6.79 & 5.99 & 2.89 & 2.17 & $10^{-1}$ \\
\hline 10.00 & 34.82 & 30.40 & 13.84 & 10.38 & 34.79 & 30.38 & 13.82 & 10.37 & 34.81 & 30.38 & 13.82 & 10.37 & $10^{-2}$ \\
\hline
\end{tabular}


TABLE XV: Neutrino flux $\left(\mathrm{m}^{-2} \mathrm{sec}^{-1} \mathrm{sr}^{-1} \mathrm{GeV}^{-1}\right)$ for $-0.3 \geq \cos \theta_{z}>-0.4$

\begin{tabular}{|c|c|c|c|c|c|c|c|c|c|c|c|c|c|}
\hline & & Kam & nioka & & & Sudl & bury & & & Gran & I Sasso & & \\
\hline$E_{\nu}(\mathrm{GeV})$ & $\nu_{\mu}$ & $\bar{\nu}_{\mu}$ & $\nu_{e}$ & $\bar{\nu}_{e}$ & $\nu_{\mu}$ & $\bar{\nu}_{\mu}$ & $\nu_{e}$ & $\bar{\nu}_{e}$ & $\nu_{\mu}$ & $\bar{\nu}_{\mu}$ & $\nu_{e}$ & $\bar{\nu}_{e}$ & Norm \\
\hline .1000 & 7.18 & 7.17 & 3.71 & 3.07 & 8.78 & 8.78 & 4.58 & 3.73 & 8.11 & 8.10 & 4.24 & $4 \quad 3.44$ & $10^{3}$ \\
\hline .1259 & 5.50 & 5.50 & 2.86 & 2.37 & 6.74 & 6.76 & 3.55 & 2.88 & 6.22 & 6.23 & 3.27 & $7 \quad 2.66$ & $10^{3}$ \\
\hline .1585 & 40.89 & 40.91 & 21.30 & 17.66 & 50.16 & 50.40 & 26.41 & 21.47 & 46.30 & 46.41 & 24.36 & 6 19.70 & $10^{2}$ \\
\hline .1995 & 29.09 & 29.20 & 15.31 & 12.68 & 35.81 & 36.09 & 18.98 & 15.46 & 32.97 & 33.19 & 17.52 & 214.22 & $10^{2}$ \\
\hline .2512 & 19.83 & 20.01 & 10.61 & 8.83 & 24.43 & 24.74 & 13.20 & 10.78 & 22.41 & 22.73 & 12.15 & $5 \quad 9.95$ & $10^{2}$ \\
\hline .3162 & 13.17 & 13.26 & 7.13 & 5.95 & 16.12 & 16.36 & 8.88 & 7.27 & 14.82 & 15.04 & 48.16 & $6 \quad 6.67$ & $10^{2}$ \\
\hline .3981 & 8.55 & 8.56 & 4.67 & 3.90 & 10.43 & 10.54 & 5.79 & 4.75 & 9.58 & 9.67 & 5.30 & 4.34 & $10^{2}$ \\
\hline .5012 & 5.45 & 5.43 & 2.99 & 2.50 & 6.64 & 6.65 & 3.67 & 3.02 & 6.07 & 6.09 & 3.36 & 2.76 & $10^{2}$ \\
\hline .6310 & 34.37 & 33.92 & 18.70 & 15.68 & 41.40 & 41.06 & 22.86 & 18.77 & 37.87 & 37.71 & 20.94 & 417.20 & $10^{1}$ \\
\hline .7943 & 21.22 & 20.80 & 11.49 & 9.60 & 25.25 & 24.92 & 13.93 & 11.37 & 23.18 & 22.92 & 12.79 & 910.44 & $10^{1}$ \\
\hline 1.000 & 12.85 & 12.50 & 6.94 & 5.74 & 15.09 & 14.82 & 8.31 & 6.71 & 13.91 & 13.64 & 7.62 & 26.20 & $10^{1}$ \\
\hline 1.259 & 7.70 & 7.38 & 4.10 & 3.36 & 8.84 & 8.61 & 4.84 & 3.87 & 8.22 & 7.96 & 4.45 & $5 \quad 3.61$ & $10^{1}$ \\
\hline 1.585 & 45.18 & 42.97 & 23.64 & 19.23 & 50.86 & 49.00 & 27.46 & 21.72 & 47.82 & 45.75 & 25.47 & 720.34 & 1 \\
\hline 1.995 & 25.92 & 24.5 & 13.28 & 10.68 & 28.78 & 27.29 & 15.12 & 11.83 & 27.30 & 25.79 & 14.23 & 311.10 & 1 \\
\hline 2.512 & 14.74 & 13.60 & 7.30 & 5.74 & 15.99 & 14.81 & 8.10 & 6.25 & 15.27 & 14.15 & 7.67 & $7 \quad 5.96$ & 1 \\
\hline 3.162 & 8.18 & 7.39 & 3.88 & 3.00 & 8.70 & 7.90 & 4.21 & 3.21 & 8.38 & 7.62 & 4.00 & 3.10 & 1 \\
\hline 3.981 & 44.14 & 39.47 & 19.87 & 15.28 & 46.34 & 41.46 & 21.26 & 16.05 & 45.10 & 40.31 & 20.37 & 715.59 & $10^{-1}$ \\
\hline 5.012 & 23.39 & 20.55 & 9.98 & 7.58 & 24.19 & 21.23 & 10.41 & 7.81 & 23.76 & 20.82 & 10.10 & 7.70 & $10^{-1}$ \\
\hline 6.310 & 12.26 & 10.60 & 4.89 & 3.69 & 12.42 & 10.73 & 4.96 & 3.72 & 12.33 & 10.64 & 4.91 & $1 \quad 3.71$ & $10^{-1}$ \\
\hline 7.943 & 63.48 & 54.22 & 23.37 & 17.57 & 63.29 & 54.02 & 23.28 & 17.52 & 63.40 & 54.12 & 23.36 & 17.53 & $10^{-2}$ \\
\hline 10.00 & 32.33 & 27.26 & 10.90 & 8.19 & 32.30 & 27.23 & 10.89 & 8.18 & 32.32 & 27.25 & 10.90 & 8.18 & $10^{-2}$ \\
\hline
\end{tabular}


TABLE XVI: Neutrino flux $\left(\mathrm{m}^{-2} \mathrm{sec}^{-1} \mathrm{sr}^{-1} \mathrm{GeV}^{-1}\right)$ for $-0.4 \geq \cos \theta_{z}>-0.5$

\begin{tabular}{|c|c|c|c|c|c|c|c|c|c|c|c|c|c|}
\hline & & $\mathrm{Ka}$ & aioka & & & Sud & lbury & & & Gran & Sasso & & \\
\hline$E_{\nu}(\mathrm{GeV})$ & $\nu_{\mu}$ & $\bar{\nu}_{\mu}$ & $\nu_{e}$ & $\bar{\nu}_{e}$ & $\nu_{\mu}$ & $\bar{\nu}_{\mu}$ & $\nu_{e}$ & $\bar{\nu}_{e}$ & $\nu_{\mu}$ & $\bar{\nu}_{\mu}$ & $\nu_{e}$ & $\bar{\nu}_{e}$ & Norm \\
\hline .1000 & 6.38 & 6.38 & 3.32 & 2.77 & 6.82 & 6.81 & 3.57 & 2.96 & 6.92 & 6.92 & 3.64 & $4 \quad 2.98$ & $10^{3}$ \\
\hline .1259 & 5.00 & 5.00 & 2.61 & 2.18 & 5.35 & 5.36 & 2.80 & 2.32 & 5.42 & 5.44 & 2.86 & $6 \quad 2.34$ & $10^{3}$ \\
\hline .1585 & 38.12 & 38.05 & 19.76 & 16.47 & 40.84 & 40.86 & 21.25 & 17.61 & 41.36 & 41.44 & 21.68 & 17.72 & $10^{2}$ \\
\hline .1995 & 27.88 & 27.83 & 14.45 & 12.08 & 29.94 & 29.95 & 15.61 & 12.94 & 30.21 & 30.29 & 15.87 & 713.00 & $10^{2}$ \\
\hline .2512 & 19.43 & 19.48 & 10.21 & 8.58 & 20.90 & 21.01 & 11.08 & 9.18 & 20.99 & 21.18 & 11.19 & $9 \quad 9.21$ & $10^{2}$ \\
\hline .3162 & 13.07 & 13.11 & 6.99 & 5.87 & 14.07 & 14.16 & 7.56 & 6.27 & 14.11 & 14.26 & 7.64 & $4 \quad 6.28$ & $10^{2}$ \\
\hline .3981 & 8.59 & 8.59 & 4.61 & 3.88 & 9.22 & 9.25 & 4.98 & 4.15 & 9.22 & 9.27 & 5.02 & 24.14 & $10^{2}$ \\
\hline .5012 & 5.54 & 5.50 & 2.95 & 2.49 & 5.92 & 5.90 & 3.19 & 2.66 & 5.89 & 5.87 & 3.19 & 92.65 & $10^{2}$ \\
\hline .6310 & 34.96 & 34.36 & 18.46 & 15.56 & 37.28 & 36.80 & 19.95 & 16.62 & 36.88 & 36.51 & 19.85 & 516.43 & $10^{1}$ \\
\hline .7943 & 21.58 & 21.01 & 11.35 & 9.49 & 22.94 & 22.46 & 12.18 & 10.08 & 22.63 & 22.17 & 12.10 & 9.92 & $10^{1}$ \\
\hline 1.000 & 13.04 & 12.60 & 6.81 & 5.64 & 13.82 & 13.43 & 7.27 & 5.95 & 13.57 & 13.14 & 7.18 & 85.84 & $10^{1}$ \\
\hline 1.259 & 7.74 & 7.41 & 3.97 & 3.25 & 8.18 & 7.85 & 4.24 & 3.42 & 7.95 & 7.66 & 4.14 & $4 \quad 3.35$ & $10^{1}$ \\
\hline 1.585 & 45.18 & 42.66 & 22.52 & 18.21 & 47.46 & 44.97 & 23.99 & 19.12 & 45.88 & 43.72 & 23.22 & 218.68 & 1 \\
\hline 1.995 & 25.90 & 23.98 & 12.47 & 9.92 & 26.95 & 25.18 & 13.15 & 10.36 & 26.15 & 24. & 12.72 & 210.14 & 1 \\
\hline 2.512 & 14.50 & 13.17 & 6.70 & 5.27 & 14.99 & 13.66 & 7.00 & 5.43 & 14.62 & 13.34 & 6.80 & 5.31 & 1 \\
\hline 3.162 & 7.93 & 7.07 & 3.48 & 2.70 & 8.16 & 7.27 & 3.61 & 2.78 & 7.99 & 7.11 & 3.54 & $\begin{array}{ll}4 & 2.71\end{array}$ & 1 \\
\hline 3.981 & 42.59 & 37.21 & 17.55 & 13.41 & 43.50 & 38.10 & 18.08 & 13.79 & 42.74 & 37.21 & 17.78 & 13.54 & $10^{-1}$ \\
\hline 5.012 & 22.58 & 19.26 & 8.62 & 6.54 & 22.82 & 19.50 & 8.78 & 6.62 & 22.47 & 19.29 & 8.65 & $5 \quad 6.56$ & $10^{-1}$ \\
\hline 6.310 & 11.72 & 9.83 & 4.13 & 3.11 & 11.76 & 9.86 & 4.15 & 3.11 & 11.67 & 9.85 & 4.12 & 23.11 & $10^{-1}$ \\
\hline 7.943 & 59.88 & 49.72 & 19.31 & 14.48 & 59.85 & 49.68 & 19.28 & 14.48 & 59.95 & 49.67 & 19.32 & 214.49 & $10^{-2}$ \\
\hline 10.00 & 30.52 & 25.01 & 8.88 & 6.68 & 30.51 & 25.00 & 8.88 & 6.68 & 30.53 & 25.00 & 8.88 & 86.68 & $10^{-2}$ \\
\hline
\end{tabular}


TABLE XVII: Neutrino flux $\left(\mathrm{m}^{-2} \mathrm{sec}^{-1} \mathrm{sr}^{-1} \mathrm{GeV}^{-1}\right)$ for $-0.5 \geq \cos \theta_{z}>-0.6$

\begin{tabular}{|c|c|c|c|c|c|c|c|c|c|c|c|c|c|}
\hline & & $\mathrm{Ka}$ & nioka & & & Sud & lbury & & & Gran & I Sasso & & \\
\hline$E_{\nu}(\mathrm{GeV})$ & $\nu_{\mu}$ & $\bar{\nu}_{\mu}$ & $\nu_{e}$ & $\bar{\nu}_{e}$ & $\nu_{\mu}$ & $\bar{\nu}_{\mu}$ & $\nu_{e}$ & $\bar{\nu}_{e}$ & $\nu_{\mu}$ & $\bar{\nu}_{\mu}$ & $\nu_{e}$ & $\bar{\nu}_{e}$ & Norm \\
\hline .1000 & 6.34 & 6.33 & 3.30 & 2.78 & 5.61 & 5.62 & 2.93 & 2.49 & 6.38 & 6.41 & 3.36 & $\begin{array}{ll}6 & 2.81\end{array}$ & $10^{3}$ \\
\hline .1259 & 5.04 & 5.04 & 2.63 & 2.20 & 4.48 & 4.49 & 2.33 & 1.99 & 5.09 & 5.11 & 2.67 & $\begin{array}{ll}7 & 2.23\end{array}$ & $10^{3}$ \\
\hline .1585 & 39.19 & 39.05 & 20.17 & 16.92 & 34.95 & 34.89 & 17.96 & 15.29 & 39.67 & 39.66 & 20.62 & 217.07 & $10^{2}$ \\
\hline .1995 & 29.21 & 29.07 & 14.97 & 12.57 & 26.20 & 26.15 & 13.39 & 11.38 & 29.67 & 29.62 & 15.35 & 512.70 & $10^{2}$ \\
\hline .2512 & 20.67 & 20.62 & 10.70 & 8.99 & 18.66 & 18.70 & 9.63 & 8.17 & 21.02 & 21.05 & 10.98 & 9.12 & $10^{2}$ \\
\hline .3162 & 14.06 & 14.02 & 7.36 & 6.18 & 12.79 & 12.78 & 6.68 & 5.66 & 14.26 & 14.32 & 7.55 & $5 \quad 6.27$ & $10^{2}$ \\
\hline .3981 & 9.27 & 9.20 & 4.87 & 4.10 & 8.49 & 8.45 & 4.46 & 3.77 & 9.38 & 9.39 & 4.98 & $\begin{array}{l}8.14 \\
\end{array}$ & $10^{2}$ \\
\hline .5012 & 5.94 & 5.86 & 3.11 & 2.62 & 5.49 & 5.45 & 2.87 & 2.42 & 6.02 & 5.98 & 3.16 & $6 \quad 2.64$ & $10^{2}$ \\
\hline .6310 & 37.15 & 36.46 & 19.32 & 16.18 & 34.85 & 34.15 & 18.01 & 15.10 & 37.64 & 37.06 & 19.64 & 416.38 & $10^{1}$ \\
\hline .7943 & 22.72 & 22.11 & 11.69 & 9.67 & 21.58 & 20.98 & 11.02 & 9.21 & 22.95 & 22.43 & 11.88 & $\begin{array}{l}8.79 \\
\end{array}$ & $10^{1}$ \\
\hline 1.000 & 13.59 & 13.09 & 6.89 & 5.63 & 13.04 & 12.63 & 6.56 & 5.45 & 13.66 & 13.26 & 6.97 & $7 \quad 5.67$ & $10^{1}$ \\
\hline 1.259 & 7.93 & 7.59 & 3.96 & 3.20 & 7.74 & 7.37 & 3.79 & 3.10 & 7.96 & 7.64 & 13.96 & 3.21 & $10^{1}$ \\
\hline 1.585 & 45.68 & 42.98 & 22.09 & 17.59 & 45.04 & 42.01 & 21.38 & 17.22 & 45.58 & 42.92 & 22.09 & 917.62 & 1 \\
\hline 1.995 & 25.90 & 23. & 11.92 & 9.36 & 25.64 & 23.45 & 11.73 & 9.28 & 25.68 & 23 & 11.99 & 99.34 & 1 \\
\hline 2.512 & 14.28 & 12.76 & 6.20 & 4.83 & 14.29 & 12.77 & 6.21 & 4.82 & 14.25 & 12.79 & 6.24 & $\begin{array}{ll}4 & 4.82\end{array}$ & 1 \\
\hline 3.162 & 7.7 & 6.76 & 3.16 & 2.43 & 7.78 & 6.80 & 3.17 & 2.43 & 7.74 & 6.77 & 73.14 & $\begin{array}{ll}4 & 2.42\end{array}$ & 1 \\
\hline 3.981 & 41.39 & 35.40 & 15.72 & 11.94 & 41.56 & 35.46 & 15.71 & 11.99 & 41.14 & 35.16 & 15.46 & 11.79 & $10^{-1}$ \\
\hline 5.012 & 21.67 & 18.13 & 7.53 & 5.69 & 21.84 & 18.16 & 7.58 & 5.71 & 21.59 & 18.04 & 17.45 & 5.64 & $10^{-1}$ \\
\hline 6.310 & 11.18 & 9.18 & 3.53 & 2.66 & 11.24 & 9.19 & 3.55 & 2.66 & 11.18 & 9.18 & 3.52 & 2.65 & $10^{-1}$ \\
\hline 7.943 & 57.20 & 46.31 & 16.30 & 12.27 & 57.10 & 46.27 & 16.28 & 12.26 & 57.20 & 46.32 & 16.32 & 12.28 & $10^{-2}$ \\
\hline 10.00 & 29.11 & 23.29 & 7.42 & 5.60 & 29.09 & 23.29 & 7.42 & 5.60 & 29.11 & 23.29 & 7.42 & 5.61 & $10^{-2}$ \\
\hline
\end{tabular}


TABLE XVIII: Neutrino flux $\left(\mathrm{m}^{-2} \mathrm{sec}^{-1} \mathrm{sr}^{-1} \mathrm{GeV}^{-1}\right)$ for $-0.6 \geq \cos \theta_{z}>-0.7$

\begin{tabular}{|c|c|c|c|c|c|c|c|c|c|c|c|c|c|}
\hline & & $\mathrm{Ka}$ & aioka & & & Sud & lbury & & & Gran & Sasso & & \\
\hline$E_{\nu}(\mathrm{GeV})$ & $\nu_{\mu}$ & $\bar{\nu}_{\mu}$ & $\nu_{e}$ & $\bar{\nu}_{e}$ & $\nu_{\mu}$ & $\bar{\nu}_{\mu}$ & $\nu_{e}$ & $\bar{\nu}_{e}$ & $\nu_{\mu}$ & $\bar{\nu}_{\mu}$ & $\nu_{e}$ & $\bar{\nu}_{e}$ & Norm \\
\hline .1000 & 6.59 & 6.58 & 3.49 & 2.87 & 4.70 & 4.71 & 2.41 & 2.17 & 6.04 & 6.05 & 3.16 & $5 \quad 2.69$ & $10^{3}$ \\
\hline .1259 & 5.31 & 5.30 & 2.79 & 2.30 & 3.83 & 3.84 & 1.96 & 1.76 & 4.89 & 4.90 & 2.55 & $5 \quad 2.18$ & $10^{3}$ \\
\hline .1585 & 41.77 & 41.56 & 21.59 & 17.76 & 30.47 & 30.44 & 15.32 & 13.64 & 38.71 & 38.75 & 19.86 & 6 16.85 & $10^{2}$ \\
\hline .1995 & 31.43 & 31.23 & 16.11 & 13.25 & 23.33 & 23.23 & 11.61 & 10.28 & 29.43 & 29.41 & 14.95 & 512.61 & $10^{2}$ \\
\hline .2512 & 22.33 & 22.30 & 11.54 & 9.51 & 17.01 & 16.93 & 8.50 & 7.51 & 21.20 & 21.17 & 10.82 & 29.09 & $10^{2}$ \\
\hline .3162 & 15.10 & 15.14 & 7.89 & 6.51 & 11.85 & 11.81 & 5.98 & 5.25 & 14.52 & 14.50 & 7.49 & 6.27 & $10^{2}$ \\
\hline .3981 & 9.87 & 9.85 & 5.16 & 4.27 & 8.01 & 7.92 & 4.04 & 3.52 & 9.57 & 9.54 & 4.96 & $5 \quad 4.14$ & $10^{2}$ \\
\hline .5012 & 6.28 & 6.20 & 3.25 & 2.70 & 5.28 & 5.16 & 2.63 & 2.28 & 6.13 & 6.07 & 3.16 & $6 \quad 2.63$ & $10^{2}$ \\
\hline .6310 & 38.81 & 38.07 & 19.93 & 16.42 & 33.67 & 32.79 & 16.68 & 14.24 & 38.16 & 37.44 & 19.49 & 916.14 & $10^{1}$ \\
\hline .7943 & 23.39 & 22.71 & 11.80 & 9.68 & 20.98 & 20.26 & 10.25 & 8.62 & 23.12 & 22.44 & 11.63 & 9.56 & $10^{1}$ \\
\hline 1.000 & 13.80 & 13.21 & 6.77 & 5.54 & 12.79 & 12.17 & 6.11 & 5.06 & 13.67 & 13.12 & 6.73 & 35.48 & $10^{1}$ \\
\hline 1.259 & 7.99 & 7.54 & 3.81 & 3.07 & 7.58 & 7.13 & 3.54 & 2.89 & 7.91 & 7.49 & 3.81 & 13.04 & $10^{1}$ \\
\hline 1.585 & 45.39 & 42.20 & 20.99 & 16.56 & 43.85 & 40.55 & 19.79 & 15.94 & 45.03 & 41.80 & 20.81 & 16.40 & 1 \\
\hline 1.995 & 25.40 & 23.07 & 11.17 & 8.69 & 24.91 & 22 . & 10.68 & 8.50 & 25.23 & 22 & 11.00 & 8.59 & 1 \\
\hline 2.512 & 14.02 & 12.33 & 5.71 & 4.41 & 13.90 & 12.22 & 5.60 & 4.39 & 13.87 & 12.25 & 5.67 & $7 \quad 4.38$ & 1 \\
\hline 3.162 & 7.59 & 6.48 & 2.86 & 2.19 & 7.56 & 6.46 & 2.84 & 2.19 & 7.50 & 6.46 & 2.84 & $\begin{array}{ll}4 & 2.17\end{array}$ & 1 \\
\hline 3.981 & 40.24 & 33.60 & 14.03 & 10.61 & 40.13 & 33.42 & 13.88 & 10.60 & 39.84 & 33.49 & 13.82 & 210.53 & $10^{-1}$ \\
\hline 5.012 & 21.00 & 17.19 & 6.63 & 5.02 & 20.96 & 17.15 & 6.62 & 4.99 & 20.79 & 17.08 & 6.55 & $5 \quad 5.00$ & $10^{-1}$ \\
\hline 6.310 & 10.80 & 8.69 & 3.07 & 2.32 & 10.79 & 8.70 & 3.08 & 2.31 & 10.74 & 8.66 & 3.06 & 2.32 & $10^{-1}$ \\
\hline 7.943 & 55.00 & 43.67 & 14.04 & 10.56 & 55.02 & 43.67 & 14.02 & 10.57 & 55.09 & 43.73 & 14.05 & 510.56 & $10^{-2}$ \\
\hline 10.00 & 27.98 & 21.96 & 6.34 & 4.78 & 27.98 & 21.96 & 6.33 & 4.79 & 28.00 & 21.97 & 6.34 & $4 \quad 4.79$ & $10^{-2}$ \\
\hline
\end{tabular}


TABLE XIX: Neutrino flux $\left(\mathrm{m}^{-2} \mathrm{sec}^{-1} \mathrm{sr}^{-1} \mathrm{GeV}^{-1}\right)$ for $-0.7 \geq \cos \theta_{z}>-0.8$

\begin{tabular}{|c|c|c|c|c|c|c|c|c|c|c|c|c|c|}
\hline & & $\mathrm{Ka}$ & nioka & & & Sud & lbury & & & Gran & I Sasso & & \\
\hline$E_{\nu}(\mathrm{GeV})$ & $\nu_{\mu}$ & $\bar{\nu}_{\mu}$ & $\nu_{e}$ & $\bar{\nu}_{e}$ & $\nu_{\mu}$ & $\bar{\nu}_{\mu}$ & $\nu_{e}$ & $\bar{\nu}_{e}$ & $\nu_{\mu}$ & $\bar{\nu}_{\mu}$ & $\nu_{e}$ & $\bar{\nu}_{e}$ & Norm \\
\hline .1000 & 6.27 & 6.26 & 3.32 & 2.76 & 4.44 & 4.43 & 2.27 & 2.08 & 5.35 & 5.37 & 72.80 & $0 \quad 2.43$ & $10^{3}$ \\
\hline .1259 & 5.10 & 5.10 & 2.68 & 2.22 & 3.66 & 3.66 & 1.86 & 1.69 & 4.40 & 4.41 & 2.29 & $\begin{array}{ll}9 & 1.97\end{array}$ & $10^{3}$ \\
\hline .1585 & 40.67 & 40.48 & 20.90 & 17.28 & 29.57 & 29.45 & 14.71 & 13.29 & 35.25 & 35.23 & 17.97 & 715.36 & $10^{2}$ \\
\hline .1995 & 30.96 & 30.77 & 15.69 & 12.96 & 22.95 & 22.77 & 11.24 & 10.09 & 27.09 & 27.00 & 13.60 & 011.60 & $10^{2}$ \\
\hline .2512 & 22.18 & 22.11 & 11.28 & 9.32 & 16.90 & 16.74 & 8.27 & 7.37 & 19.67 & 19.60 & 9.88 & 8.41 & $10^{2}$ \\
\hline .3162 & 15.07 & 15.03 & 7.72 & 6.40 & 11.88 & 11.75 & 5.84 & 5.16 & 13.55 & 13.53 & 6.85 & $5 \quad 5.79$ & $10^{2}$ \\
\hline .3981 & 9.85 & 9.79 & 5.05 & 4.19 & 8.06 & 7.92 & 3.95 & 3.45 & 9.00 & 8.94 & $4 \quad 4.55$ & $5 \quad 3.83$ & $10^{2}$ \\
\hline .5012 & 6.25 & 6.16 & 3.17 & 2.62 & 5.31 & 5.17 & 2.57 & 2.22 & 5.81 & 5.71 & 2.90 & 2.44 & $10^{2}$ \\
\hline .6310 & 38.58 & 37.63 & 19.26 & 15.84 & 33.85 & 32.65 & 16.20 & 13.85 & 36.38 & 35.47 & 717.83 & 314.90 & $10^{1}$ \\
\hline .7943 & 23.20 & 22.40 & 11.31 & 9.26 & 20.96 & 20.05 & 9.89 & 8.32 & 22.12 & 21.34 & 10.65 & $5 \quad 8.80$ & $10^{1}$ \\
\hline 1.000 & 13.63 & 13.02 & 6.46 & 5.26 & 12.64 & 11.98 & 5.84 & 4.83 & 13.12 & 12.48 & 6.19 & 95.05 & $10^{1}$ \\
\hline 1.259 & 7.88 & 7.39 & 3.62 & 2.90 & 7.48 & 6.96 & 3.32 & 2.72 & 7.63 & 7.16 & 3.48 & $\begin{array}{ll}3 & 2.81\end{array}$ & $10^{1}$ \\
\hline 1.585 & 44.73 & 41.02 & 19.62 & 15.50 & 43.12 & 39.38 & 18.42 & 14.82 & 43.68 & 40.04 & 18.94 & 415.17 & 1 \\
\hline 1.995 & 24.95 & 22.32 & 10.28 & 8.01 & 24.29 & 21.74 & 9.91 & 7.81 & 24.57 & 21 & 10.00 & 7.92 & 1 \\
\hline 2.512 & 13.67 & 11.89 & 5.24 & 4.03 & 13.46 & 11.70 & 5.11 & 3.96 & 13.53 & 11.72 & 25.15 & $5 \quad 3.99$ & 1 \\
\hline 3.162 & 73.70 & 62.17 & 26.05 & 19.83 & 73.02 & 61.62 & 25.52 & 19.58 & 73.11 & 61 & 25. & 419.61 & $10^{-1}$ \\
\hline 3.981 & 39.08 & 32.01 & 12.59 & 9.54 & 38.75 & 31.85 & 12.39 & 9.47 & 38.85 & 31.81 & 12.43 & 9.42 & $10^{-1}$ \\
\hline 5.012 & 20.33 & 16.28 & 5.91 & 4.45 & 20.25 & 16.20 & 5.84 & 4.46 & 20.28 & 16.26 & 5.85 & 4.40 & $10^{-1}$ \\
\hline 6.310 & 10.44 & 8.23 & 2.71 & 2.04 & 10.43 & 8.21 & 2.70 & 2.04 & 10.44 & 8.24 & 12.70 & 2.03 & $10^{-}$ \\
\hline 7.943 & 53.18 & 41.52 & 12.25 & 9.24 & 53.20 & 41.56 & 12.26 & 9.23 & 53.20 & 41.51 & 12.26 & 9.25 & $10^{-2}$ \\
\hline 10.00 & 27.03 & 20.85 & 5.48 & 4.16 & 27.03 & 20.86 & 5.49 & 4.15 & 27.03 & 20.85 & 5.49 & 4.16 & $10^{-2}$ \\
\hline
\end{tabular}


TABLE XX: Neutrino flux $\left(\mathrm{m}^{-2} \mathrm{sec}^{-1} \mathrm{sr}^{-1} \mathrm{GeV}^{-1}\right)$ for $-0.8 \geq \cos \theta_{z}>-0.9$

\begin{tabular}{|c|c|c|c|c|c|c|c|c|c|c|c|c|c|}
\hline & & Kan & ioka & & & Sudl & oury & & & Gran & Sasso & & \\
\hline$E_{\nu}(\mathrm{GeV})$ & $\nu_{\mu}$ & $\bar{\nu}_{\mu}$ & $\nu_{e}$ & $\bar{\nu}_{e}$ & $\nu_{\mu}$ & $\bar{\nu}_{\mu}$ & $\nu_{e}$ & $\bar{\nu}_{e}$ & $\nu_{\mu}$ & $\bar{\nu}_{\mu}$ & $\nu_{e}$ & $\bar{\nu}_{e}$ & Norm \\
\hline .1000 & 5.54 & 5.54 & 2.94 & 2.47 & 5.14 & 5.12 & 2.70 & 2.30 & 5.09 & 5.08 & 2.69 & 2.27 & $10^{3}$ \\
\hline .1259 & 4.57 & 4.56 & 2.39 & 2.01 & 4.23 & 4.22 & 2.20 & 1.86 & 4.19 & 4.19 & 2.19 & 1.85 & $10^{3}$ \\
\hline .1585 & 36.78 & 36.55 & 18.76 & 15.71 & 34.07 & 33.78 & 17.26 & 14.57 & 33.82 & 33.61 & 17.20 & 14.46 & $10^{2}$ \\
\hline .1995 & 28.27 & 28.07 & 14.15 & 11.82 & 26.20 & 25.90 & 13.02 & 10.97 & 26.07 & 25.83 & 12.99 & 10.91 & $10^{2}$ \\
\hline .2512 & 20.41 & 20.32 & 10.19 & 8.52 & 18.96 & 18.76 & 9.40 & 7.92 & 18.89 & 18.77 & 9.38 & 7.90 & $10^{2}$ \\
\hline .3162 & 13.97 & 13.90 & 7.00 & 5.86 & 12.97 & 12.87 & 6.46 & 5.45 & 12.97 & 12.87 & 6.46 & 5.43 & $10^{2}$ \\
\hline .3981 & 9.19 & 9.11 & 4.59 & 3.84 & 8.54 & 8.44 & 4.24 & 3.57 & 8.56 & 8.47 & 4.25 & 3.57 & $10^{2}$ \\
\hline .5012 & 5.87 & 5.78 & 2.88 & 2.41 & 5.46 & 5.35 & 2.67 & 2.24 & 5.49 & 5.39 & 2.68 & 2.26 & $10^{2}$ \\
\hline .6310 & 36.56 & 35.51 & 17.51 & 14.56 & 34.07 & 33.01 & 16.26 & 13.60 & 34.36 & 33.28 & 16.38 & 13.73 & $10^{1}$ \\
\hline .7943 & 22.19 & 21.26 & 10.36 & 8.49 & 20.71 & 19.86 & 9.62 & 7.98 & 20.94 & 19.99 & 9.73 & 8.10 & $10^{1}$ \\
\hline 1.000 & 13.13 & 12.41 & 5.96 & 4.81 & 12.31 & 11.66 & 5.55 & 4.54 & 12.48 & 11.75 & 5.63 & 4.63 & $10^{1}$ \\
\hline 1.259 & 7.61 & 7.06 & 3.32 & 2.67 & 7.21 & 6.67 & 3.10 & 2.53 & 7.33 & 6.76 & 3.15 & 2.55 & $10^{1}$ \\
\hline 1.585 & 43.32 & 39.24 & 17.93 & 14.29 & 41.44 & 37.47 & 16.89 & 13.65 & 41.94 & 38.04 & 17.17 & 13.70 & 1 \\
\hline 1.995 & 24.2 & 21.38 & 9.39 & 7.34 & 23.38 & 20.64 & 8.96 & 7.13 & 23.49 & 20.8 & 9.06 & 7.15 & 1 \\
\hline 2.512 & 13.29 & 11.39 & 4.77 & 3.68 & 12.96 & 11.12 & 4.61 & 3.60 & 13.04 & 11.20 & 4.63 & 3.62 & 1 \\
\hline 3.162 & 71.67 & 59.50 & 23.60 & 17.93 & 70.44 & 58.7 & 23.04 & 17.61 & 71.02 & 59.0 & 23.02 & 17.77 & $10^{-1}$ \\
\hline 3.981 & 37.92 & 30.62 & 11.35 & 8.51 & 37.53 & 30.47 & 11.18 & 8.42 & 37.74 & 30.55 & 11.13 & 8.49 & $10^{-1}$ \\
\hline 5.012 & 19.71 & 15.58 & 5.27 & 3.99 & 19.69 & 15.61 & 5.24 & 3.95 & 19.67 & 15.51 & 5.21 & 3.95 & $10^{-1}$ \\
\hline 6.310 & 101.37 & 78.87 & 23.98 & 18.22 & 101.56 & 79.06 & 23.97 & 18.14 & 101.34 & 78.58 & 23.93 & 18.08 & $10^{-2}$ \\
\hline 7.943 & 51.76 & 39.78 & 10.83 & 8.16 & 51.74 & 39.7 & 10.84 & 8.17 & 51.76 & 39.81 & 10.84 & 8.18 & $10^{-2}$ \\
\hline 10.00 & 26.26 & 19.97 & 4.84 & 3.67 & 26.25 & 19.96 & 4.84 & 3.67 & 26.26 & 19.97 & 4.84 & 3.67 & $10^{-2}$ \\
\hline
\end{tabular}


TABLE XXI: Neutrino flux $\left(\mathrm{m}^{-2} \mathrm{sec}^{-1} \mathrm{sr}^{-1} \mathrm{GeV}^{-1}\right)$ for $-0.9 \geq \cos \theta_{z} \geq-1.0$

\begin{tabular}{|c|c|c|c|c|c|c|c|c|c|c|c|c|c|}
\hline & & Kam & aioka & & & Sudl & lbury & & & Gran & I Sasso & & \\
\hline$E_{\nu}(\mathrm{GeV})$ & $\nu_{\mu}$ & $\bar{\nu}_{\mu}$ & $\nu_{e}$ & $\bar{\nu}_{e}$ & $\nu_{\mu}$ & $\bar{\nu}_{\mu}$ & $\nu_{e}$ & $\bar{\nu}_{e}$ & $\nu_{\mu}$ & $\bar{\nu}_{\mu}$ & $\nu_{e}$ & $\bar{\nu}_{e}$ & Norm \\
\hline .1000 & 5.36 & 5.36 & 2.82 & 2.41 & 6.51 & 6.49 & 3.49 & 2.83 & 5.82 & 5.81 & L 3.11 & $1 \quad 2.57$ & $10^{3}$ \\
\hline .1259 & 44.49 & 44.41 & 23.09 & 19.67 & 53.59 & 53.54 & 28.38 & 22.91 & 48.10 & 47.97 & 25.22 & 20.90 & $10^{2}$ \\
\hline .1585 & 36.08 & 35.90 & 18.15 & 15.43 & 43.23 & 42.80 & 22.09 & 17.86 & 38.90 & 38.63 & 19.75 & 5 16.29 & $10^{2}$ \\
\hline .1995 & 27.96 & 27.73 & 13.72 & 11.65 & 33.09 & 32.63 & 16.50 & 13.36 & 29.95 & 29.65 & 14.84 & 412.22 & $10^{2}$ \\
\hline .2512 & 0.35 & 20.18 & 9.94 & 8.41 & 23.57 & 23.38 & 11.77 & 9.53 & 21.52 & 21.34 & 10.63 & 38.78 & $10^{2}$ \\
\hline .3162 & 13.98 & 13.83 & 6.83 & 5.75 & 15.83 & 15.75 & 7.94 & 6.44 & 14.60 & 14.48 & 7.23 & 3.97 & $10^{2}$ \\
\hline .3981 & 9.21 & 9.07 & 4.47 & 3.76 & 10.21 & 10.10 & 5.08 & 4.14 & 9.51 & 9.40 & 4.67 & $\begin{array}{ll}7 & 3.87\end{array}$ & $10^{2}$ \\
\hline .5012 & 5.89 & 5.75 & 2.80 & 2.35 & 6.38 & 6.25 & 3.11 & 2.54 & 6.01 & 5.88 & 2.89 & 92.40 & $10^{2}$ \\
\hline .6310 & 36.55 & 35.27 & 16.99 & 14.15 & 38.73 & 37.52 & 18.39 & 15.07 & 36.89 & 35.69 & 17.36 & 14.32 & $10^{1}$ \\
\hline .7943 & 22.11 & 21.01 & 9.97 & 8.23 & 22.97 & 21.99 & 10.59 & 8.61 & 22.11 & 21.10 & 10.09 & 98.26 & $10^{1}$ \\
\hline 1.000 & 13.05 & 12.20 & 5.68 & 4.63 & 13.35 & 12.59 & 5.93 & 4.76 & 12.96 & 12.19 & 5.68 & 84.62 & $10^{1}$ \\
\hline 1.259 & 7.53 & 6.94 & 3.15 & 2.53 & 7.61 & 7.04 & 3.23 & 2.58 & 7.46 & 6.90 & 3.13 & 32.51 & $10^{1}$ \\
\hline 1.585 & 42.71 & 38.46 & 16.89 & 13.34 & 42.73 & 38.55 & 17.08 & 13.54 & 42.33 & 38.10 & 16.75 & 513.31 & 1 \\
\hline 1.9 & 23.85 & 20.80 & 8.75 & 6.83 & 23.72 & 20.77 & 8.79 & 6.88 & 23.65 & 20 & 8.70 & 6.8 & 1 \\
\hline 2.512 & 13.08 & 11.05 & 4.42 & 3.41 & 12.98 & 11.00 & 4.40 & 3.40 & 12.96 & 10.95 & 4.38 & $3 \quad 3.38$ & 1 \\
\hline 3.162 & 70.3 & $57.6^{\prime}$ & 21.6 & 16.52 & 69.94 & 57.36 & 21.49 & 16.37 & 69.84 & 57 & 321 & 16.35 & $10^{-1}$ \\
\hline 3.981 & 37.14 & 29.61 & 10.32 & 7.78 & 36.98 & 29.49 & 10.22 & 7.73 & 37.02 & 29.59 & 10.23 & 37.77 & $10^{-1}$ \\
\hline 5.012 & 19.31 & 15.02 & 4.77 & 3.59 & 19.20 & 15.00 & 4.74 & $\begin{array}{l}3.59 \\
\end{array}$ & 19.29 & 15.03 & 4.75 & $5 \quad 3.60$ & $10^{-1}$ \\
\hline 6.310 & 99.03 & 75.85 & 21.63 & 16.31 & 98.70 & 75.84 & 21.61 & 16.31 & 99.03 & 75.87 & 21.64 & 416.32 & $10^{-}$ \\
\hline 7.943 & 50.36 & 38.23 & 9.70 & 7.32 & 50.41 & 38.23 & 9.70 & 7.32 & 50.37 & 38.23 & 9.70 & 7.32 & $10^{-2}$ \\
\hline 10.00 & 25.57 & 19.22 & 4.31 & 3.27 & 25.58 & 19.21 & 4.31 & 3.27 & 25.57 & 19.21 & 4.31 & $1 \quad 3.27$ & $10^{-2}$ \\
\hline
\end{tabular}




\section{APPENDIX B: ATMOSPHERIC NEUTRINO FLUX ABOVE 10 GEV}

Here we tabulate the atmospheric neutrino flux calculated in this work for neutrino energies above $10 \mathrm{GeV}$ in Tables XXII-XXV. The atmospheric neutrino flux could also be calculated using Eq. A1,

Note, we tabulate one kind of neutrino flux in one table independently of the observation site, for the down going directions. The neutrino flux for upward going direction is obtained using the mirror symmetry of the atmospheric neutrino flux:

$$
\phi_{\nu}(-\cos \theta)=\phi_{\nu}(\cos \theta)
$$

valid in the energy region where the rigidity cutoff does not affect the neutrino flux.

TABLE XXII: $\nu_{\mu}$ flux $\left(\mathrm{m}^{-2} \mathrm{sec}^{-1} \mathrm{sr}^{-1} \mathrm{GeV}^{-1}\right)$ above $10 \mathrm{GeV}$

\begin{tabular}{|c|c|c|c|c|c|c|c|c|c|c|c|}
\hline \multirow[b]{2}{*}{$E_{\nu}(\mathrm{GeV})$} & \multicolumn{10}{|c|}{$\cos \theta_{z}$} & \multirow[b]{2}{*}{ Norm } \\
\hline & -.9 & $.9-.8$ & $3-.7$ & -.6 & $.6-.5$ & $.5-.4$ & $.4-.3$ & $.3-.2$ & $.2-.1$ & $.1-.0$ & \\
\hline & 7 & 5 & 3 & 9 & 911 & 3.052 & 2 & 2 & 28 & 1 & \\
\hline & .295 & 1.331 & 1.370 & .419 & 1.479 & 1.554 & 646 & 1.778 & 1.964 & 2.166 & $10^{-1}$ \\
\hline & 65 & 0.673 & 0.694 & 0 & 0.751 & 0.789 & 840 & 0006 & 1.009 & .121 & $10^{-}$ \\
\hline & 3.297 & 3.397 & 3 & 3 & 3.811 & 4 & $\$ 9$ & 4. & 5.154 & 0.001 & $10^{-}$ \\
\hline 10 & 659 & 1.7 & 1 & & 1.930 & 2.0 & & 9 & 1 & 7 & 10 \\
\hline & 831 & 0.858 & 0.891 & 0.931 & 0.974 & 1.033 & 1.100 & 1.197 & 1.340 & 1.542 & $10^{-}$ \\
\hline $.981 \times 10^{1}$ & 4.14 & 201 & 4.46 & 4.663 & 4.898 & 5.205 & 5.572 & 6.091 & 6.852 & 7.935 & $10^{-}$ \\
\hline & 2.055 & 2.136 & 2.225 & 2.329 & 2.457 & 2.612 & 2.819 & 3.085 & 3.482 & 4.0 .01 & $10^{-3}$ \\
\hline & 01 & 1.056 & 1 & 1 & 12 & 1.3 & & 1. & & 2.0 & 10 \\
\hline $10^{1}$ & 400 & 0 & & 6 & 9 & 3 & 0 & 0.783 & 0.897 & 4 & 10 \\
\hline $1.000 \times 10^{2}$ & 2.443 & 2.551 & 2.679 & 2.838 & 3.012 & 3.248 & 3.541 & 3.930 & 4.524 & 5.345 & $10^{-4}$ \\
\hline $10^{2}$ & 1.194 & 1.253 & 1.315 & 1.394 & 1.487 & 1.606 & 1.761 & 1.967 & 2.259 & 2.676 & $10^{-4}$ \\
\hline & 0.58 & 0.611 & 0.643 & 0.684 & 0.732 & 0.790 & 0.869 & 0.979 & 29 & 1.338 & 10 \\
\hline 1 & 031 & 9 & 3 & 0 & 3 & 3 & 0 & 4. & 9 & 6.676 & $10^{-5}$ \\
\hline $2.512 \times 10^{2}$ & 1.371 & 1.439 & 1.521 & 1.621 & 1.732 & 1.897 & 2.092 & 2.384 & 2.785 & 3.322 & $10^{-5}$ \\
\hline $3.162 \times 10^{2}$ & 0.658 & 0.695 & 0.737 & 0.786 & 0.844 & 0.923 & 1.022 & 1.168 & 1.378 & 1.646 & $10^{-5}$ \\
\hline $3.981 \times 10^{2}$ & 3.146 & 3.328 & 3.547 & 3.792 & 4.096 & 4.482 & 4.988 & 5.700 & 6.771 & 8.124 & $10^{-6}$ \\
\hline
\end{tabular}




\begin{tabular}{|c|c|c|c|c|c|c|c|c|c|c|c|}
\hline & 49 & - & 696 & 1.81 & 975 & $2.1^{\prime}$ & 2.425 & 6 & 3 & 3.990 & \\
\hline & & & & & & & & & & & \\
\hline & 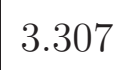 & & .807 & & & & & & & & \\
\hline & 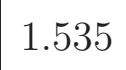 & & & & & & & & & & \\
\hline & 705 & 9 & 825 & 5 & 1 & & & & & & \\
\hline & 320 & 247 & 8 & 0 & 0 & & & & & & \\
\hline & 441 & 1.568 & 1 & 1.908 & 2 & & & & & & \\
\hline & & & & & & & & & & & \\
\hline & 285 & 0.312 & 0.346 & 87 & 0.4 & 8 & 0 & & 15 & 1.1 & \\
\hline $10^{3}>$ & 251 & 375 & 530 & & 1.9 & & & & 0 & & \\
\hline & 518 & ר 60 & 0.675 & 0750 & 0.878 & & 2 & & & & \\
\hline & م. & 0.264 & 0 & 0.335 & 0.3 & & & & & 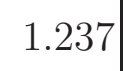 & \\
\hline 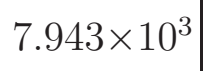 & 1.032 & 1.156 & 284 & 1.473 & 1.694 & 2.021 & .466 & 6 & 4.304 & 5.6 & 10 \\
\hline (5) & 44 & & 0.5 & o & 0.7 & & 1 & & & & \\
\hline
\end{tabular}

TABLE XXIII: $\bar{\nu}_{\mu}$ flux $\left(\mathrm{m}^{-2} \mathrm{sec}^{-1} \mathrm{sr}^{-1} \mathrm{GeV}^{-1}\right)$ above $10 \mathrm{GeV}$

\begin{tabular}{|c|c|c|c|c|c|c|c|c|c|c|c|}
\hline \multirow[b]{2}{*}{$E_{\nu}(\mathrm{GeV})$} & \multicolumn{10}{|c|}{$\cos \theta_{z}$} & \multirow[b]{2}{*}{ Norm } \\
\hline & -.9 & $.9-.8$ & $8-.7$ & $.7-.6$ & $.6-.5$ & $.5-.4$ & $.4-.3$ & $.3-.2$ & $.2-.1$ & $.1-.0$ & \\
\hline & 921 & 1.997 & 085 & 2.196 & 2.329 & 2.501 & 2.725 & 3.038 & 3.480 & 3.925 & $10^{-1}$ \\
\hline 1.2 & .965 & .002 & 046 & 1.103 & .169 & 1.255 & 369 & 1.531 & .768 & .030 & $10^{-1}$ \\
\hline $1.585 \times 10^{1}$ & 484 & 503 & 524 & 0.553 & 5 & 0.630 & 8 & 769 & 0.898 & .047 & $10^{-1}$ \\
\hline $1.995 \times 10^{1}$ & 2.419 & .521 & 2.628 & 2.774 & 2.936 & 3.157 & 3.445 & 3.869 & 4.526 & 5.334 & $10^{-2}$ \\
\hline $2.512 \times 10$ & 1.206 & 1.257 & 1.313 & 1.388 & 1.470 & 1.578 & 1.726 & 1.939 & 2.276 & 2.706 & $10^{-2}$ \\
\hline 3.16 & 0.599 & 0.624 & 0.654 & 0.692 & 0.734 & 0.788 & 0.866 & 0.970 & 1.144 & 1.378 & $10^{-2}$ \\
\hline & 2.960 & 092 & 253 & 3.433 & 667 & 3.934 & 4.341 & 4.876 & 12 & 7.037 & \\
\hline $5.012 \times 10^{1}$ & 1.456 & 1.523 & 1.603 & 1.699 & 1.815 & 1.958 & 2.157 & 2.430 & 2.869 & 3.549 & $10^{-3}$ \\
\hline $6.310 \times 10^{1}$ & 0.713 & 0.746 & 0.784 & 0.836 & 0.892 & 0.970 & 1.067 & 1.203 & 1.431 & 1.768 & $10^{-3}$ \\
\hline $10^{1}$ & 3.472 & 3.653 & 3.847 & 4.076 & 4.393 & 4.787 & 5.296 & 5.998 & 7.149 & 8.824 & $10^{-4}$ \\
\hline $1.000 \times 10^{2}$ & 1.685 & 1.777 & 1.872 & 1.987 & 2.151 & 2.349 & 2.610 & 2.971 & 3.547 & 4.404 & $10^{-4}$ \\
\hline $1.259 \times 10^{2}$ & 0.814 & 0.857 & 0.903 & 0.971 & 1.044 & 1.144 & 1.273 & 1.459 & 1.746 & 2.184 & $10^{-4}$ \\
\hline $1.585 \times 10^{2}$ & 0.390 & 0.412 & 0.437 & 0.470 & 0.506 & 0.555 & 0.620 & 0.717 & 0.859 & 1.071 & $10^{-4}$ \\
\hline
\end{tabular}




\begin{tabular}{|c|c|c|c|c|c|c|c|c|c|c|c|}
\hline $995 \times 10^{2}$ & 1.875 & 1.976 & 2.104 & 259 & 2.444 & 2.684 & 3.006 & 3.503 & 4.197 & 5.235 & $10^{-5}$ \\
\hline $512 \times 10^{2}$ & 0.898 & 0.943 & 1.007 & 1.083 & 1.173 & 1.296 & 1.450 & 1.692 & 2.037 & 2.561 & $10^{-5}$ \\
\hline $162 \times 10^{2}$ & 0.425 & 0.449 & 0.482 & 0518 & 0562 & 0.622 & 0.699 & 0.814 & 0.992 & 1 & $10^{-5}$ \\
\hline $981 \times 10^{2}$ & 2.007 & 2.136 & 2.288 & .469 & 691 & 2.975 & 3.364 & .928 & 4.804 & 6 & $10^{-6}$ \\
\hline $012 \times 10^{2}$ & 0.945 & 1.011 & 1.078 & 1.169 & 1.283 & 1.422 & 1.613 & 1.893 & 2.311 & 2.908 & $10^{-6}$ \\
\hline$\times 10^{2}$ & 0.441 & 0.472 & 0.508 & 0.551 & 0.608 & 0.675 & 0.770 & 0.903 & 1.110 & 1.397 & $10^{-6}$ \\
\hline $943 \times 10^{2}$ & 2.045 & 2.189 & 2.374 & .578 & 2.855 & 3.190 & 3.651 & 4.310 & 5.324 & 6.682 & $10^{-7}$ \\
\hline $000 \times 10^{3}$ & 0.941 & 1.012 & 1.096 & .198 & 1.331 & 1.500 & 1.724 & .054 & 13 & 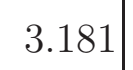 & $10^{-7}$ \\
\hline $259 \times 10^{3}$ & 0.428 & 0.463 & 0.505 & 0.555 & 0.617 & 0.700 & 0.811 & 0.968 & 1.208 & 1.514 & $10^{-7}$ \\
\hline $.585 \times 10^{3}$ & 1.930 & 2.095 & 2.298 & 2.534 & 2.839 & 3.244 & 3.777 & 4.548 & 5.717 & 7.207 & $10^{-8}$ \\
\hline $995 \times 10^{3}$ & 0.863 & 0.939 & 1.032 & 1.143 & 1.295 & 1.489 & 1.743 & 2.129 & 2.692 & 3.416 & $10^{-8}$ \\
\hline $512 \times 10$ & 0.379 & 0.418 & 0.461 & 0.515 & 0.584 & 0.675 & 0.799 & 0.983 & 1.260 & 1.602 & $10^{-8}$ \\
\hline $162 \times 10^{3}$ & 1.672 & 1.846 & 2.046 & .293 & 2.610 & 3.044 & 3.641 & 4.523 & 5.860 & 7.5 & $10^{-9}$ \\
\hline $.981 \times 10^{3}$ & 0.736 & 0.810 & 0.900 & 1.014 & 1.158 & 1.361 & 1.646 & 2.069 & 2.704 & 3.526 & $10^{-9}$ \\
\hline $.012 \times 10^{3}$ & 0.316 & 0.352 & 0.394 & 0.449 & 0.510 & 0.600 & 0.734 & 0.931 & 1.238 & 1.627 & $10^{-9}$ \\
\hline $310 \times 10^{3}$ & 1.363 & 1.534 & 1.714 & 1.946 & 2.232 & 2.662 & 3.225 & 4.149 & 5.645 & 7.456 & $10^{-10}$ \\
\hline $7.943 \times 10^{3}$ & 0.593 & 0.665 & 0.741 & 0.832 & 0.974 & 1.185 & 1.417 & 1.846 & 2.556 & 3.404 & $10^{-10}$ \\
\hline $1.000 \times 10^{4}$ & 0.256 & 0.281 & 0.318 & 0.361 & 0.424 & 0.516 & 0.634 & 0.820 & 1.140 & 1.541 & $10^{-10}$ \\
\hline
\end{tabular}


TABLE XXIV: $\nu_{e}$ flux $\left(\mathrm{m}^{-2} \mathrm{sec}^{-1} \mathrm{sr}^{-1} \mathrm{GeV}^{-1}\right)$ above $10 \mathrm{GeV}$

\begin{tabular}{|c|c|c|c|c|c|c|c|c|c|c|c|}
\hline \multirow[b]{2}{*}{$E_{\nu}(\mathrm{GeV})$} & \multicolumn{10}{|c|}{$\cos \theta_{z}$} & \multirow[b]{2}{*}{ Norm } \\
\hline & 1.-.9 & $.9-.8$ & $.8-.7$ & $.7-.6$ & $.6-.5$ & $.5-.4$ & $.4-.3$ & $.3-.2$ & $.2-.1$ & $.1-.0$ & \\
\hline $1.000 \times 10^{1}$ & 0.431 & 0.484 & 0.548 & 0.634 & 0.742 & 0.888 & 1.090 & 1.383 & 1.798 & 2.242 & $10^{-1}$ \\
\hline $1.259 \times 10^{1}$ & 0.191 & 0.215 & 0.245 & 0.284 & 0.335 & 0.404 & 0.503 & 0.650 & 0.871 & 1.131 & $10^{-1}$ \\
\hline $1.585 \times 10^{1}$ & 0.848 & 0.951 & 1.088 & 1.263 & 1.497 & 1.819 & 2.296 & 3.015 & 4.177 & 5.624 & $10^{-2}$ \\
\hline $1.995 \times 10^{1}$ & 0.374 & 0.421 & 0.479 & 0.561 & 0.665 & 0.817 & 1.033 & 1.386 & 1.968 & 2.759 & $10^{-2}$ \\
\hline $2.512 \times 10^{1}$ & 0.166 & 0.187 & 0.212 & 0.248 & 0.295 & 0.363 & 0.463 & 0.630 & 0.917 & 1.335 & $10^{-2}$ \\
\hline $162 \times 10^{1}$ & 0.740 & 0.831 & 0.942 & 1.102 & 1.308 & 1.604 & 2.073 & 2.841 & 4.240 & 6.414 & $10^{-3}$ \\
\hline $3.981 \times 10^{1}$ & 0.331 & 0.371 & 0.419 & 0.489 & 0.580 & 0.714 & 0.926 & 1.284 & 1.945 & 3.080 & $10^{-3}$ \\
\hline $5.012 \times 10^{1}$ & 0.151 & 0.167 & 0.187 & 0.216 & 0.256 & 0.316 & 0.410 & 0.570 & 0.881 & 1.451 & $10^{-3}$ \\
\hline $6.310 \times 10^{1}$ & 0.685 & 0.752 & 0.842 & 0.959 & 1.130 & 1.390 & 1.804 & 2.501 & 3.952 & 6.684 & $10^{-4}$ \\
\hline $7.943 \times 10^{1}$ & 0.303 & 0.340 & 0.373 & 0.429 & 0.504 & 0.611 & 0.792 & 1.110 & 1.770 & 3.062 & $10^{-4}$ \\
\hline $1.000 \times 10^{2}$ & 0.138 & 0.154 & 0.169 & 0.194 & 0.226 & 0.272 & 0.350 & 0.488 & 0.781 & 1.388 & $10^{-4}$ \\
\hline $1.259 \times 10^{2}$ & 0.649 & 0.704 & 0.771 & 0.883 & 1.018 & 1.219 & 1.552 & 2.122 & 3.391 & 6.202 & $10^{-5}$ \\
\hline $1.585 \times$ & 0.290 & 0.323 & 0.347 & 0.395 & 0.461 & 0.546 & 0.686 & 0.938 & 1.480 & .740 & $10^{-5}$ \\
\hline $1.995 \times 10^{2}$ & 0.132 & 0.148 & 0.161 & 0.180 & 0.209 & 0.245 & 0.305 & 0.414 & 0.649 & 1.203 & $10^{-5}$ \\
\hline $2.512 \times 10^{2}$ & 0.617 & 0.673 & 0.758 & 0.828 & 0.947 & 1.103 & 1.367 & 1.812 & 2.853 & 5.262 & $10^{-6}$ \\
\hline $3.162 \times 10^{2}$ & 0.285 & 0.308 & 0.344 & 0.377 & 0.431 & 0.500 & 0.616 & 0.803 & 1.249 & 2.283 & $10^{-6}$ \\
\hline $3.981 \times 10^{2}$ & 1.299 & 1.406 & 1.541 & 1.714 & 1.957 & 2.275 & 2.767 & 3.600 & 5.457 & 9.861 & $10^{-7}$ \\
\hline $5.012 \times 10^{2}$ & 0.591 & 0.640 & 0.700 & 0.782 & 0.888 & 1.033 & 1.238 & 1.613 & 2.387 & 4.247 & $10^{-7}$ \\
\hline $6.310 \times 10^{2}$ & 0.268 & 0.289 & 0.320 & 0.354 & 0.406 & 0.467 & 0.555 & 0.715 & 1.049 & 1.825 & $10^{-7}$ \\
\hline $7.943 \times 10^{2}$ & 1.204 & 1.298 & 1.441 & 1.609 & 1.836 & 2.132 & 2.532 & 3.251 & 4.645 & 7.852 & $10^{-8}$ \\
\hline $1.000 \times 10^{3}$ & 0.536 & 0.583 & 0.639 & 0.732 & 0.814 & 0.973 & 1.161 & 1.498 & 2.076 & 3.387 & $10^{-8}$ \\
\hline $1.259 \times 10^{3}$ & 0.237 & 0.262 & 0.285 & 0.327 & 0.361 & 0.435 & 0.521 & 0.667 & 0.934 & 1.464 & $10^{-8}$ \\
\hline $1.585 \times 10^{3}$ & 1.037 & 1.155 & 1.268 & 1.427 & 1.652 & 1.935 & 2.327 & 3.011 & 4.176 & 6.411 & $10^{-9}$ \\
\hline $1.995 \times 10^{3}$ & 0.453 & 0.501 & 0.560 & 0.624 & 0.752 & 0.859 & 1.042 & 1.365 & 1.859 & 2.816 & $10^{-9}$ \\
\hline $2.512 \times 10^{3}$ & 0.199 & 0.217 & 0.248 & 0.281 & 0.319 & 0.378 & 0.463 & 0.588 & 0.830 & 1.217 & $10^{-9}$ \\
\hline $3.162 \times 10^{3}$ & 0.872 & 0.954 & 1.073 & 1.216 & 1.382 & 1.651 & 2.051 & 2.620 & 3.683 & 5.416 & $10^{-10}$ \\
\hline $3.981 \times 10^{3}$ & 0.377 & 0.422 & 0.458 & 0.517 & 0.626 & 0.722 & 0.897 & 1.199 & 1.647 & 2.483 & $10^{-10}$ \\
\hline
\end{tabular}




\begin{tabular}{|c|c|c|c|c|c|c|c|c|c|c|c|}
\hline $012 \times 10^{3}$ & 16 & 0.183 & 0.201 & .236 & 0.275 & 0.321 & 0.383 & 0.518 & 0.759 & 1.114 & $10^{-10}$ \\
\hline$\times 10^{3}$ & 716 & 0.781 & 0.843 & 020 & 180 & 1.465 & 1.692 & 2.282 & 5 & 4. & $10^{-11}$ \\
\hline 0 & 305 & 0.333 & 0.353 & 415 & 0505 & 0.647 & 0.753 & 2 & & & $10^{-11}$ \\
\hline $1.000 \times 10^{4}$ & 233 & 1.428 & 637 & .773 & 2.170 & 2.604 & 143 & 900 & 88 & 2 & $10^{-12}$ \\
\hline
\end{tabular}

TABLE XXV: $\bar{\nu}_{e}$ flux $\left(\mathrm{m}^{-2} \mathrm{sec}^{-1} \mathrm{sr}^{-1} \mathrm{GeV}^{-1}\right)$ above $10 \mathrm{GeV}$

\begin{tabular}{|c|c|c|c|c|c|c|c|c|c|c|c|}
\hline \multirow[b]{2}{*}{$E_{\nu}(\mathrm{GeV})$} & \multicolumn{10}{|c|}{$\cos \theta_{z}$} & \multirow[b]{2}{*}{ Norm } \\
\hline & 1.-.9 & $.9-.8$ & $.8-.7$ & $.7-.6$ & $.6-.5$ & $.5-.4$ & $.4-.3$ & $.3-.2$ & $.2-.1$ & $.1-.0$ & \\
\hline $1.000 \times 10^{1}$ & 0.327 & 0.367 & 0.416 & 0.478 & 0.560 & 0.668 & 0.819 & 1.038 & 1.352 & 1.687 & $10^{-1}$ \\
\hline $1.259 \times 10^{1}$ & 1.465 & 1.639 & 1.858 & 2.153 & 2.531 & 3.051 & 3.779 & 4.880 & 6.539 & 8.490 & $10^{-2}$ \\
\hline $1.585 \times 10^{1}$ & 0.654 & 0.733 & 0.830 & 0.962 & 1.134 & 1.379 & 1.731 & 2.269 & 3.153 & 4.241 & $10^{-2}$ \\
\hline $1.995 \times 10^{1}$ & 0.291 & 0.327 & 0.372 & 0.431 & 0.509 & 0.622 & 0.787 & 1.047 & 1.494 & 2.081 & $10^{-2}$ \\
\hline $2.512 \times 10^{1}$ & 0.130 & 0.146 & 0.167 & 0.192 & 0.228 & 0.279 & 0.355 & 0.478 & 0.698 & 1.010 & $10^{-2}$ \\
\hline $3.162 \times 10^{1}$ & 0.589 & 0.652 & 0.746 & 0.855 & 1.021 & 1.251 & 1.599 & 2.175 & 3.239 & 4.890 & $10^{-3}$ \\
\hline $3.981 \times 10^{1}$ & 0.267 & 0.296 & 0.337 & 0.386 & 0.457 & 0.562 & 0.721 & 0.990 & 1.496 & 2.362 & $10^{-3}$ \\
\hline $5.012 \times 10^{1}$ & 0.121 & 0.134 & 0.152 & 0.173 & 0.204 & 0.250 & 0.323 & 0.446 & 0.681 & 1.116 & $10^{-3}$ \\
\hline $6.310 \times 10^{1}$ & 0.550 & 0.602 & 0.684 & 0.769 & 0.910 & 1.104 & 1.432 & 1.978 & 3.057 & 5.166 & $10^{-4}$ \\
\hline $7.943 \times 10^{1}$ & 0.250 & 0.277 & 0.311 & 0.344 & 0.404 & 0.493 & 0.633 & 0.871 & 1.364 & 2.391 & $10^{-4}$ \\
\hline $1.000 \times 10^{2}$ & 0.115 & 0.126 & 0.141 & 0.156 & 0.181 & 0.220 & 0.283 & 0.385 & 0.605 & 1.086 & $10^{-4}$ \\
\hline $1.259 \times 10^{2}$ & 0.536 & 0.570 & 0.634 & 0.717 & 0.820 & 0.985 & 1.267 & 1.706 & 2.678 & 4.847 & $10^{-5}$ \\
\hline $1.585 \times 10^{2}$ & 0.245 & 0.261 & 0.288 & 0.324 & 0.374 & 0.441 & 0.556 & 0.749 & 1.186 & 2.162 & $10^{-5}$ \\
\hline $1.995 \times 10^{2}$ & 1.113 & 1.209 & 1.307 & 1.454 & 1.686 & 1.981 & 2.448 & 3.312 & 5.199 & 9.476 & $10^{-6}$ \\
\hline $2.512 \times 10^{2}$ & 0.505 & 0.555 & 0.596 & 0.656 & 0.756 & 0.893 & 1.100 & 1.481 & 2.264 & 4.107 & $10^{-6}$ \\
\hline $3.162 \times 10^{2}$ & 0.228 & 0.249 & 0.274 & 0.302 & 0.346 & 0.404 & 0.500 & 0.660 & 0.995 & 1.806 & $10^{-6}$ \\
\hline $3.981 \times 10^{2}$ & 1.033 & 1.129 & 1.246 & 1.385 & 1.583 & 1.836 & 2.246 & 2.946 & 4.378 & 7.861 & $10^{-7}$ \\
\hline $5.012 \times 10^{2}$ & 0.470 & 0.514 & 0.559 & 0.625 & 0.716 & 0.832 & 1.006 & 1.318 & 1.926 & 3.373 & $10^{-7}$ \\
\hline $6.310 \times 10^{2}$ & 0.212 & 0.228 & 0.252 & 0.280 & 0.321 & 0.372 & 0.458 & 0.590 & 0.856 & 1.458 & $10^{-7}$ \\
\hline $7.943 \times 10^{2}$ & 0.936 & 1.011 & 1.133 & 1.256 & 1.441 & 1.660 & 2.046 & 2.697 & 3.818 & 6.328 & $10^{-8}$ \\
\hline $1.000 \times 10^{3}$ & 0.408 & 0.451 & 0.503 & 0.562 & 0.646 & 0.746 & 0.904 & 1.227 & 1.697 & 2.738 & $10^{-8}$ \\
\hline $1.259 \times 10^{3}$ & 0.180 & 0.200 & 0.221 & 0.248 & 0.287 & 0.337 & 0.412 & 0.529 & 0.748 & 1.178 & $10^{-8}$ \\
\hline $1.585 \times 10^{3}$ & 0.796 & 0.870 & 0.967 & 1.099 & 1.272 & 1.508 & 1.846 & 2.330 & 3.312 & 5.101 & $10^{-9}$ \\
\hline
\end{tabular}




\begin{tabular}{l|cccccccccc|c}
$1.995 \times 10^{3}$ & 0.347 & 0.379 & 0.423 & 0.485 & 0.566 & 0.659 & 0.806 & 1.054 & 1.473 & 2.236 & $10^{-9}$ \\
$2.512 \times 10^{3}$ & 1.499 & 1.665 & 1.824 & 2.101 & 2.514 & 2.847 & 3.542 & 4.578 & 6.517 & 9.874 & $10^{-10}$ \\
$3.162 \times 10^{3}$ & 0.643 & 0.717 & 0.774 & 0.898 & 1.086 & 1.259 & 1.537 & 2.057 & 2.870 & 4.396 & $10^{-10}$ \\
$3.981 \times 10^{3}$ & 0.277 & 0.305 & 0.330 & 0.388 & 0.456 & 0.557 & 0.663 & 0.915 & 1.270 & 1.919 & $10^{-10}$ \\
$5.012 \times 10^{3}$ & 1.197 & 1.328 & 1.434 & 1.723 & 1.928 & 2.333 & 2.939 & 3.614 & 5.617 & 7.958 & $10^{-11}$ \\
$6.310 \times 10^{3}$ & 0.507 & 0.557 & 0.618 & 0.726 & 0.859 & 1.003 & 1.299 & 1.576 & 2.278 & 3.389 & $10^{-11}$ \\
$7.943 \times 10^{3}$ & 0.212 & 0.227 & 0.263 & 0.305 & 0.381 & 0.453 & 0.551 & 0.739 & 0.932 & 1.522 & $10^{-11}$ \\
$1.000 \times 10^{4}$ & 0.910 & 0.973 & 1.133 & 1.435 & 1.529 & 1.997 & 2.215 & 2.971 & 4.573 & 6.825 & $10^{-12}$ \\
\hline \hline
\end{tabular}

